\title{
Smooth and Robust Solutions for Dirichlet Boundary Control of Fluid-Solid Conjugate Heat Transfer Problems
}

\author{
Yan Yan
}

Submitted in partial fulfillment of the

requirements for the degree

of Doctor of Philosophy

in the Graduate School of Arts and Sciences

\section{COLUMBIA UNIVERSITY}


(C) 2015

Yan Yan

All Rights Reserved 


\section{ABSTRACT \\ Smooth and Robust Solutions for Dirichlet Boundary
Control of Fluid-Solid Conjugate Heat Transfer Problems}

Yan Yan

This work offers new computational methods for the optimal control of the conjugate heat transfer (CHT) problem in thermal science. Conjugate heat transfer has many important industrial applications, such as heat exchange processes in power plants and cooling in electronic packaging industry, and has been a staple of computational methods in thermal science for many years. This work considers the Dirichlet boundary control of fluid-solid CHT problems. The CHT system falls into the category of multi-physics problems. Its domain typically consists of two parts, namely, a solid region subject to thermal heating or cooling and a conjugate fluid region responsible for thermal convection transport. These two different physical systems are strongly coupled through the thermal boundary condition at the fluid-solid interface. The objective in the CHT boundary control problem is to select optimally the fluid inflow profile that minimizes an objective function that involves the sum of the mismatch between the temperature distribution in the system and a prescribed temperature profile and the cost of the control. This objective is realized by minimizing a nonlinear objective function of the boundary control and the fluid temperature variables, subject to partial differential equations (PDE) constraints governed by the coupled heat diffusion equation in the solid region and mass, momentum and energy conservation equations in the fluid region.

Although CHT has received extensive attention as a forward problem, the optimal Dirichlet velocity boundary control for the coupled CHT process to our knowledge is only very sparsely studied analytically or computationally in the literature [131]. Therefore, in Part I, we describe the formulation of the optimal control problem and introduce the building blocks for the finite element modeling of the CHT problem, namely, the diffusion equation for the solid temperature, the convection-diffusion equation for the fluid temperature, the incompressible viscous Navier-Stokes equations for the fluid velocity and pressure, and the model verification of CHT simulations. 
In Part II, we provide theoretical analysis to explain the nonsmoothness issue which has been observed in this study and in Dirichlet boundary control of Navier-Stokes flows by other scientists. Based on these findings, we use either explicit or implicit numerical smoothing to resolve the nonsmoothness issue. Moreover, we use the numerical continuation on regularization parameters to alleviate the difficulty of locating the global minimum in one shot for highly nonlinear optimization problems even when the initial guess is far from optimal. Two suites of numerical experiments have been provided to demonstrate the feasibility, effectiveness and robustness of the optimization scheme.

In Part III, we demonstrate the strategy of achieving parallel scalable algorithms for CHT models in Simulations of Reactor Thermal Hydraulics. Our motivation originates from the observation that parallel processing is necessary for optimal control problems of very large scale, when the simulation of the underlying physics (or PDE constraints) involves millions or billions of degrees of freedom. To achieve the overall scalability of optimal control problems governed by PDE constraints, scalable components that resolve the PDE constraints and their adjoints are the key. In this Part, first we provide the strategy of designing parallel scalable solvers for each building blocks of the CHT modeling, namely, for the discrete diffusive operator, the discrete convection-diffusion operator, and the discrete Navier-Stokes operator. Second, we demonstrate a pair of effective, robust, parallel, and scalable solvers built with collaborators for simulations of reactor thermal hydraulics. Finally, in the the section of future work, we outline the roadmap of parallel and scalable solutions for Dirichlet boundary control of fluid-solid conjugate heat transfer processes. 


\section{Table of Contents}

List of Figures $\quad$ v

List of Tables

1 Introduction 1

I Problem Formulation and Numerical Building Blocks 6

2 Problem Formulation $\quad 7$

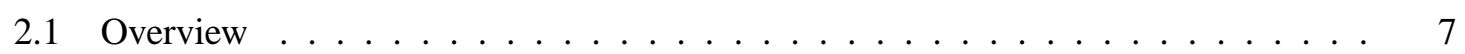

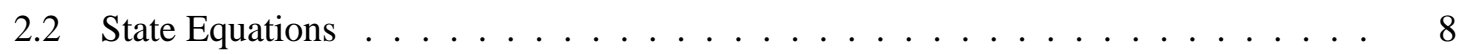

2.3 Objective Function . . . . . . . . . . . . . . . . . . . 10

3 Building Blocks for CHT simulations $\quad 12$

3.1 Sobolev Spaces . . . . . . . . . . . . . . . . . . . . . 12

3.2 Diffusion and Convection-diffusion Equations . . . . . . . . . . . . . 13

3.2 .1 Diffusion equations . . . . . . . . . . . . . . . 13

3.2.2 Convection-diffusion Equations $\ldots \ldots \ldots \ldots 14$

3.3 Incompressible Navier-Stokes Equations . . . . . . . . . . . . . . . . 18

3.4 Conjugate Heat Transfer Modeling and its Verification _ . . . . . . . . . . 22

3.4.1 Model Verification in Multiphysics Simulations . . . . . . . . . . . . 22

II Smooth and Robust Solutions for Dirichlet Boundary Control of Fluid-Solid 
4 Optimality Conditions in Infinite Dimensional Spaces 29

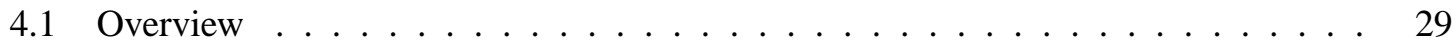

4.2 Weak Form of the State Equations $\ldots \ldots \ldots \ldots$

4.3 First Order Optimality Conditions ． . . . . . . . . . . . . . . . . . . . . 31

4.4 Necessity of $\boldsymbol{\beta}_{\mathbf{2}}>0$ in the Continuous Problem . . . . . . . . . . . . . 34

5 Discretization of the State and the Objective 35

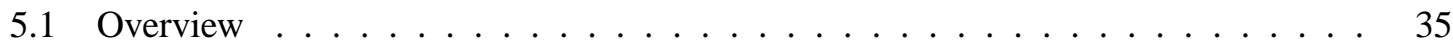

5.2 Discrete State Equations _ . . . . . . . . . . . . . . . . . . . 35

5.3 Necessity of the Smoothness Term in the Discrete Objective Function . . . . . . . 40

6 Numerical Optimization Algorithms 42

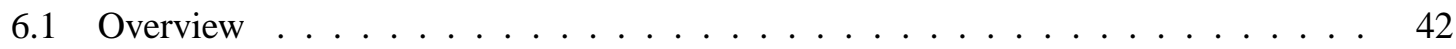

6.2 "Discretize Then Optimize" . . . . . . . . . . . . . . . . . . . . . . . . 43

6.3 Smoothed Steepest Descent Method . . . . . . . . . . . . . . . . . . . . . . . . 44

6.4 Limited-Memory BFGS Method . . . . . . . . . . . . . . . . . . . . . . . 48

6.5 Numerical Continuation on Regularization Parameters . . . . . . . . . . . . . 50

7 Numerical Test Models for Smooth and Robust Solutions 53

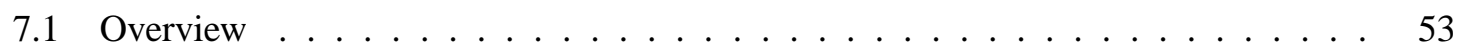

7.2 Numerical Test Problem $1 \ldots \ldots \ldots \ldots \ldots$

7.2.1 Necessity of the Regularization Term on the Control Gradient . . . . . . . 56

7.2.2 Necessity of the Smoothing Operator for the Steepest Descent Method . . . 57

7.2.3 Comparison between the Smoothed Steepest Descent Method and the LBFGS Method . . . . . . . . . . . . . . . . . . . . 59

7.2.4 Choices of the observation operator and regularization parameters $\ldots . .60$

7.3 Numerical Test Problem $2 \ldots \ldots \ldots$. . . . . . . . . . . . . . . . 64

7.3.1 Numerical Continuation on the regularization parameters . . . . . . . . . 68

7.3.2 Boundary Control of CHT flow past a square cylinder $\ldots \ldots$. . . . . 70 
III Parallel Scalable Solutions for Conjugate Heat Transfer Models in Simulations of Reactor Thermal Hydraulics $\quad 86$

9 Parallel Scalable Preconditioners for Multiphysics Simulations of CHT processes $\quad 87$

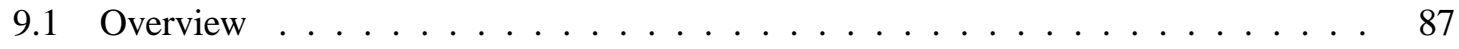

9.2 Preconditioners for Diffusion and Convection-Diffusion Equations . . . . . . . . . 88

9.3 Preconditioners for Stokes and Navier-Stokes Equations _ . . . . . . . . . . . . 89

9.3.1 Stokes Equations . . . . . . . . . . . . . . . . . . . . . . . . . . 89

$9.3 .2 \quad$ Navier-Stokes Equations . . . . . . . . . . . . . . . . . . . . . . . 91

10 Parallel Linear Solvers for Simulations of Reactor Thermal Hydraulics 93

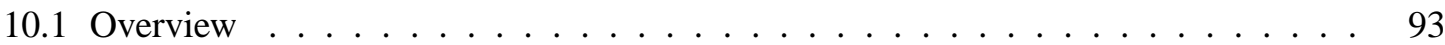

10.2 AMG Preconditioned Newton-Krylov Methods . . . . . . . . . . . . . . . . . . . 94

10.3 Scalable Data Interface and Scaling Results . . . . . . . . . . . . . . . . . . 95

11 Future Directions $\quad 98$

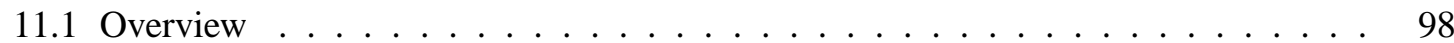

11.2 Scalable Preconditioners for the State Equations . . . . . . . . . . . . . . . . . . 99

11.3 Adaptation of the Optimization Framework to Practical Engineering Settings _ . . 100

$\begin{array}{lll}\text { IV Conclusions } & 104\end{array}$

12 Conclusions $\quad 105$

$\begin{array}{lll}\text { V Bibliography } & 107\end{array}$

$\begin{array}{ll}\text { Bibliography } & 108\end{array}$ 
B Computer Codes for Multiphysics Controls $\quad 129$

B.1 System and Software Requirements . . . . . . . . . . . . . . . . . . . 129

B.2 The Main Routine . . . . . . . . . . . . . . . . . . . . . . . . . 131

C The Lagrange-Newton-Reduced-Krylov (LNRK) Framework for Multiphysics Con$\begin{array}{ll}\text { trols } & 133\end{array}$

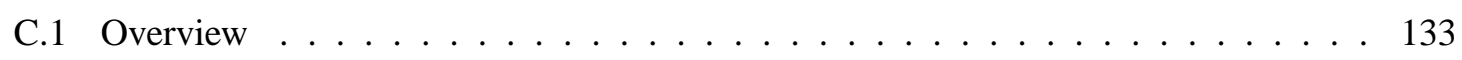

C.2 Lagrange-Newton Reduced Space Approach . . . . . . . . . . . . . . . . . . 134

C.3 Quasi-Newton Approximation for the Reduced Hessian . . . . . . . . . . . . . 137

C.4 Connections to the Implicitly Constrained Optimization Problem . . . . . . . . . . 138

C.5 Merit Functions for the Line Search Process . . . . . . . . . . . . . . . . . . . . . 141

C.6 Inexactness of the Solution Process . . . . . . . . . . . . . . . . . . . . . . 142 


\section{List of Figures}

2.1 Physical domain of the coupled fluid-solid conjugate heat transfer problem. . . . . 8

3.1 Diffusion equation on a square domain with constant forcing. . . . . . . . . . . . 15

3.2 Convection-diffusion equation on a square domain with a constant wind blowing skew to the grid. Comparison of Galerkin FEM and SUPG FEM. . . . . . . . . . 17

3.3 Q2-Q1 solution of Poiseuille channel flow with parabolic inflow boundary condition

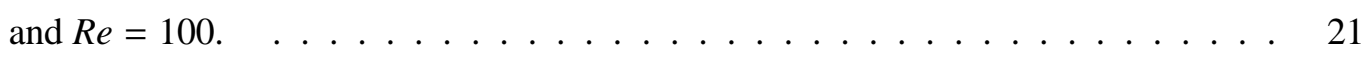

3.4 Q2-Q1-Q2 grid of the CHT problem domain. . . . . . . . . . . . 22

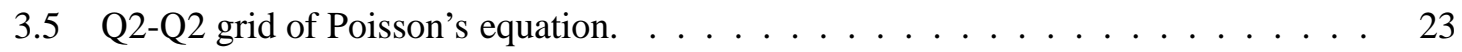

3.6 Simulation results for Poisson's equation. . . . . . . . . . . . . . . 24

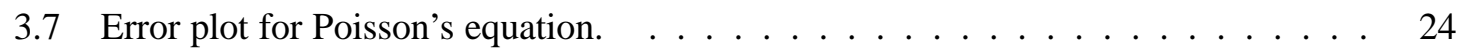

3.8 Q2-Q2 grid of the Convection-Diffusion test problem. . . . . . . . . . 26

3.9 Comparison of simulation results from the CHT code and the Q1 Galerkin code for

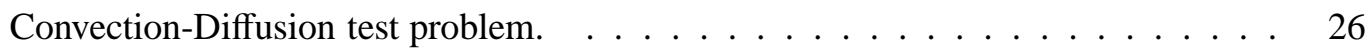

3.10 Difference of the two simulation results for the Convection-Diffusion test problem. 27

7.1 Target temperature distribution with prescribed inflow $\mathbf{g}=2\left(1.5 y-2 y^{2}, 0\right)^{T}$. The dashed line indicates the fluid-solid interface . . . . . . . . . . . . . . 55

7.2 Nonsmooth velocity solution after 100 plain Steepest Descent iterations when without smoothness control, or when $\beta_{2}=0 \ldots \ldots \ldots \ldots$. . . . . . . . 57

7.3 Nonsmooth control profiles generated without smoothness control, using $\beta_{2}=0$. There are two patterns: one for $h=1 / 24,1 / 40,1 / 60$ and the other one for the rest, and both are increasingly oscillatory as meshsize $h$ decreases. . . . . . . . . . 58 
7.4 Convergence history of the smoothed Steepest Descent method and the L-BFGS methods. . . . . . . . . . . . . . . . . . . . . . . 61

7.5 Fluid velocity solution at the last L-BFGS line search iteration. . . . . . . . . . . 61

7.6 Temperature solution at the last L-BFGS line search iteration with $\Omega_{d}=\Omega_{2} \ldots \ldots 62$

7.7 Last adjoint temperature solution of the L-BFGS method with $\Omega_{d}=\Omega_{2} \ldots \ldots$. . . 62

7.8 Last adjoint velocity solution of the L-BFGS method with $\Omega_{d}=\Omega_{2} \ldots \ldots$. . . . . . 63

7.9 Last adjoint temperature solution of the L-BFGS method with $\Omega_{d}=[0.075,1] \times\{0.75\} .65$

7.10 Last adjoint velocity solution of the L-BFGS method with $\Omega_{d}=[0.075,1] \times\{0.75\} . \quad 65$

7.11 Computed controls for both the smoothed Steepest Descent (SD) method and the LBFGS method. In the full observation (obs.) case, $\Omega_{d}=\Omega_{2}$ and $\beta_{1}=\beta_{2}=1$. In the partial observation case, $\Omega_{d}=[0.075,1] \times\{0.75\}, \beta_{1}=5 \times 10^{-2}$ and $\beta_{2}=5 \times 10^{-3}$. The exact optimal control profile is described in $(7.1) \ldots \ldots$. . . . . . . . . . 66

7.12 Computed controls for the L-BFGS method with the partial observation domain $\Omega_{d}=[0.075,1] \times\{0.75\}$. The exact optimal control profile is described in (7.1). . .

7.13 Computed controls of Numerical Test 2 using the L-BFGS method with 5-phase regularization continuation. We have set $r t o l=10^{-2}$ for the L-BFGS method to generate this plot.

7.14 Computed $x$ components of the fluid velocity in Numerical Test 2 using the L-BFGS method with 5-phase regularization continuation. We have set $r t o l=10^{-2}$ for the L-BFGS method to generate this plot. . . . . . . . . . . 7

7.15 History of the objective function value in Numerical Test 2 using the L-BFGS method with 5-phase regularization continuation. We have set $r t o l=10^{-2}$ for the L-BFGS method to generate this plot. . . . . . . . . . . . . . . . . . . 73

7.16 Computed controls of Numerical Test 2 using the L-BFGS method with 5-phase regularization continuation. The comparison is made for different starting regularization parameters $\left(\beta_{1}, \beta_{2}\right)$. The exact optimal control profile is described in (7.3). .

7.17 Computed controls of Numerical Test 2 using the L-BFGS method with 5-phase regularization continuation. The comparison is made for different reduction factors $c$ in Algorithm 6.5.1. The exact optimal control profile is described in (7.3). . . . . 
7.18 Computed controls of Numerical Test 2 using the L-BFGS method with regularization continuation. The comparison is made for different levels of white Gaussian noise added to the target temperature. The exact optimal control profile is described

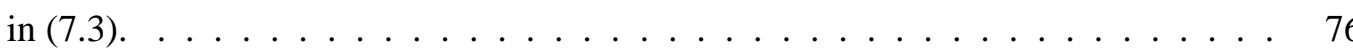

7.19 Computed controls of Numerical Test 2 using the L-BFGS method with 5-phase regularization continuation. The comparison is made for different relative tolerances in the L-BFGS method. The exact optimal control profile is described in (7.3). . . . 77

7.20 Physical domain of the flow past a square cylinder. . . . . . . . . . . . . . . 79

7.21 Target temperature in the boundary control of CHT flow past a square cylinder. . . 79

7.22 Initial temperature in the boundary control of CHT flow past a square cylinder. . . 80

7.23 Temperature induced by the computed optimal control in the boundary control of CHT flow past a square cylinder, using the L-BFGS method without regularization continuation. The relative tolerance is $r t o l=10^{-6}$. . . . . . . . . . . . 81

7.24 Temperature induced by the computed optimal control in the boundary control of CHT flow past a square cylinder, using the 2-phase L-BFGS method. The computed temperature profile in this case matches the target temperature better than the profile generated by the L-BFGS method without regularization continuation in Figure 7.23. 82

7.25 Computed controls of CHT flow past a square cylinder using the L-BFGS method with 2-phase regularization continuation. We have set $r t o l=10^{-2}$ for the L-BFGS method to generate this plot. The exact optimal control profile is described in (7.5).

10.1 Weak scaling results with full and partial-splitting-2 strategies for two-phase flow in the reactor core geometry. . . . . . . . . . . . . . . . . . . . . . 97 


\section{List of Tables}

7.1 Computed optimal objective function values using $\beta_{2}=0$. The comparison is made for different meshsizes. It is evident that the objective function values $\mathcal{J}\left(\mathbf{g}_{h}^{\star}\right)$ do not have a limit point, as the meshsize $h$ goes to 0 . This shows the need for the smoothing term in the objective function. . . . . . . . . . . . 58

7.2 Computed optimal objective function values using $\beta_{2}=1$ and the L-BFGS method with numerical smoothing. The comparison is made for different meshsizes. It is evident that the objective function values $\mathcal{J}\left(\mathbf{g}_{h}^{\star}\right)$ converge, as the meshsize $h$ goes to 0 . This demonstrate the effectiveness of the smoothing term in the objective function and the numerical smoothing operator in the optimization scheme. . . . .

7.3 Order of convergence (OC) for the smoothed Steepest Descent method and the LBFGS method. Both methods are effective and have the same OC when the numerical smoothing device is turned on. . . . . . . . . . . .

7.4 Effects of regularization on optimization (Opt.) and line search (L.s.) iterations counts for the smoothed Steepest Descent method and the L-BFGS method with the partial observation domain $\Omega_{d}=[0.075,1] \times\{0.75\} \ldots \ldots \ldots 66$

7.5 Optimization (Opt.) and line search (L.s.) iterations counts in Numerical Test 2 using the L-BFGS method with 5-phase regularization continuation. The comparison is made for different starting regularization parameters $\left(\beta_{1}, \beta_{2}\right)$. The index $i$ runs

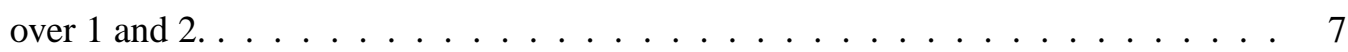


7.6 Optimization (Opt.) and line search (L.s.) iterations counts in Numerical Test 2 using the L-BFGS method with 5-phase regularization continuation. The comparison is made for different reduction factors $c$. The quality of the computed controls degrades as $c$ decreases, as shown in Figure 7.17 . . . . . . . . . . . . . . 73

7.7 Optimization (Opt.) and line search (L.s.) iterations counts in Numerical Test 2 using the L-BFGS method with 5-phase regularization continuation. The comparison is made for different relative tolerances in the L-BFGS method. . . . . . . . . . . . 74 


\section{Acknowledgments}

I would like to express my deepest thanks to Professor David E. Keyes, my Ph.D. thesis advisor, who generously gave of his time, passed on knowledge to me and taught me how to conduct research. He had led me to high performance computing and guided me through the thesis work by providing constant source of encouragement and inspirations. I would also like to express my gratitude to Professor Guillaume Bal, Professor Marc W. Spiegelman, Professor Olof B. Widlund, and Professor Pierre-David Létourneau, my thesis committee members, who has provided many valuable suggestions and comments for this thesis.

I must mention Professor Matthias Heinkenschloss and Professor Margaret H. Wright, who provided various suggestions related to this thesis work. Also, I would like to thank my RPI collaborators, Professor Michael Podowski, Professor Steven Antal, Professor Igor Bolotnov, Dr. Brian Edge and Mr. Dillon Shaver for being very supportive and collaborative on the NERI research project, Professor Edmond Chow, Dr. Barry Smith and the PETSc team for providing suggestions and support on the work "Parallel Linear Solvers for Simulations of Reactor Thermal Hydraulics", Professor Wolfgang Bangerth and the deal.II team for providing me the support and opportunity to join the Fourth deal.II Users' and Developers' Workshop, Professor Yuefan Deng for multiple inspirations, and Professor Ronald Hoppe, my master's degree advisor, who guided me to scientific computing. I am grateful to all the professors with whom I have taken courses at Columbia and NYU, and to all my colleagues and the staff members at Applied Physics and Applied Mathematics Department of Columbia University. You make this difficult journey enjoyable.

I gratefully acknowledge awards DE-FG07-07ID14889 and DE-FC02-06ER25783 from the U.S. Department of Energy for part of the research, access to computational resource at the New York Center for Computational Sciences at Stony Brook University/Brookhaven National Laboratory which is supported by the U.S. Department of Energy under Contract DE-AC02-98CH10886 
and by the State of New York, and access to resources of the National Energy Research Scientific Computing Center, which is supported by the Office of Science of the U.S. Department of Energy under Contract No. DE-AC02-05CH11231.

I thank my parents for making everything possible. 


\section{Chapter 1}

\section{Introduction}

Conjugate heat transfer (CHT) represents a process in which a conducting solid exchanges heat with a working fluid [73]. Conjugate heat transfer has many important industrial applications, such as heat exchange processes in power plants and cooling in electronic packaging, and has been a staple of computational methods in thermal science for many years $[73,89,105]$. In the meantime, with the advance in computer hardware and computational algorithms, theoretical and numerical studies of optimal control of systems governed by partial differential equations (PDE) have been highly active research areas since the founding works by Pontryagin [109] and Lions [96]. Large-scale optimal control problems for various engineering applications have attracted significant attention for a decade [3,21]. Optimal control techniques are used to automate the engineering optimization process, for instance, fluid control [1,21], shape optimization [78,99], inverse problems [10,67,68], and melting and solidification process control [77].

This work is a step in the development of practical optimal control algorithms for the CHT process, which strongly couples the fluid motion with the thermal convection transport. The optimal control of CHT has proven computationally challenging. First, the CHT system is a multiphysics system one phase of which is nonlinear, and this makes the optimization problem on top nonconvex and nonlinear. Second, properly resolving discrete governing equations of CHT can range to millions of degrees of freedom (DOF), which renders the control problem large scale. Third, like many other PDE-constrained optimization problems, the separation between the state and the control (or design) variables demands careful consideration for calculating the gradient of the objective function. In practice, the gradient computation is carried out via either the sensitivity or the adjoint 


\section{CHAPTER 1. INTRODUCTION}

approach [62]. Because of the structural similarity between the state and the adjoint (or sensitivity) equations, it is advantageous for the optimization code to reuse the solver of the state equations as either a solver or a preconditioner for the adjoint equations. These three aspects challenge generalpurpose optimization software.

The mathematical theory regarding the existence and the regularity of solutions to optimal control for Navier-Stokes equations and for convection-diffusion equations has received significant attention in the past $[62,63,65]$. Furthermore, extensive numerical experiments have been conducted for these two classes of problems $[26,57,62,63]$. Yet, to our knowledge, the optimal control problem for the coupled CHT process has not been analytically or computationally studied in the literature. In this manuscript, based on the regularity estimate for finite element solutions to the Dirichlet velocity boundary control (DVBC) of Navier-Stokes equations, we derive the regularity estimate for finite element solutions to the DVBC for CHT processes. In both cases, the regularity of the boundary control solution profile is lower than that of the full domain flow profile. This indicates the nonsmoothness of finite element approximations to the optimal boundary control solution of these two classes of DVBC problems. The nonsmoothness of the boundary control solutions to the DVBC for Navier-Stokes equations has been observed and resolved from function space perspective in $[43,70]$. In our numerical experiments, we have observed the nonsmoothness of the boundary control solutions for the CHT process. (See Figure 7.2 of Section 7.2.1.) Our strategy to resolve this nonsmoothness issue is to add regularity terms on the control gradient to both the continuous objective function and its discretization, and to use numerical smoothing on the discrete reduced gradient. In fact, for control of external fluids, the actuation devices can involve flow injection and suction. However, for some inflow boundary control problems of internal fluids, such as the DVBC of CHT processes, a smooth control that involves only flow injection is desirable.

In addition to the issue of nonsmoothness, the optimal control problem can be very large scale and demand parallel processing when the underlying simulation requires solutions of high resolution. Therefore, a parallel scalable implementation of the preconditioners, solvers and optimizers that can fully utilize the distributed computing hardware is of great interest. Our focus in this article is to build up a numerical optimization framework that generates smooth and robust approxima- 


\section{CHAPTER 1. INTRODUCTION}

tions to the optimal boundary control profile for CHT processes and to illustrate a roadmap towards parallel and scalable optimal control codes for CHT processes.

An outline of the dissertation is as follows. In Part I, we formulate the prototype problem as a PDE-constrained optimization problem in function spaces, in which the objective function represents the sum of the mismatch between the fluid temperature and a prescribed temperature distribution and the cost of the control. The constraints of the optimization problem are governed by the coupled Navier-Stokes equations in the fluid domain and the convection-diffusion equations in the fluid and the adjacent solid domain. The control device is the normal component fluid velocity at the inflow boundary. Our objective is to select optimally the inflow velocity control profile via forcing the fluid temperature to match a prescribed temperature, that is, via minimizing the objective function.

We discuss the problem formulation in Chapter 2 and the numerical building blocks for the CHT simulation in Chapter 3, namely the finite element approximation for the convection-diffusion equation and the incompressible Navier-Stokes equations. At the end of Chapter 3, we discuss the model verification of CHT simulations.

In Part II, we study a new optimization scheme that generates smooth and robust solutions for DVBC of conjugate heat transfer (CHT) processes. The solutions to the DVBC of the incompressible Navier-Stokes equations are typically nonsmooth, due to the regularity degradation of the boundary stress in the adjoint Navier-Stokes equations. This nonsmoothness is inherited by the solutions to the DVBC of CHT processes, since the CHT process couples the Navier-Stokes equations of fluid motion with the convection-diffusion equations of fluid-solid thermal interaction. Our strategy to resolve the nonsmoothness of the boundary control solution is based on two features, namely, the objective function with a regularization term on the gradient of the control profile on both the continuous and the discrete levels, and the optimization scheme with either explicit or implicit smoothing effects, such as the smoothed Steepest Descent and the Limited-memory BroydenFletcher-Goldfarb-Shanno (L-BFGS) method. Our strategy to achieve the robustness of the solution process is based on combining the smoothed optimization scheme with the numerical continuation technique on the regularization parameters. 


\section{CHAPTER 1. INTRODUCTION}

In Chapter 4, we derive the first order optimality conditions and discuss the well-posedness of the optimal control problem in infinite dimensional function spaces. In Chapter 5, we discretize the control problem with stable Galerkin Finite Element methods and discuss the issue of nonsmoothness of the boundary control solution in finite dimensional spaces. In Chapter 6, we develop the optimization scheme used in this study, namely, the smoothed Steepest Descent method and the L-BFGS method, with a thorough treatment for the nonsmoothness issue on the discrete level. At the end of Chapter 6, a variant of the L-BFGS method based on the numerical continuation on regularization parameters is developed for a robust solution strategy when the initial control profile is far from optimal. In Chapter 7, we present two suites of numerical test problems. The first one demonstrates the feasibility and effectiveness of our numerical schemes in recovering the control profile of the standard case of Poiseuille flow smoothly, and the second one demonstrates the robustness of the proposed optimization scheme for both the channel flow and the flow past a square cylinder. For this rather challenging second test problem, the optimization scheme starts from an initial control profile far from optimal and requires the numerical continuation of regularization parameters to converge to the optimal control. These two experiments demonstrate the feasibility, effectiveness and robustness of our optimization schemes. The solution strategy of Part II is general and can be applied to other large-scale optimal control problems which involve multiphysics processes and require smooth approximations to the optimal control profile.

In Part III, we discuss our strategy for parallel scalable solutions to CHT models in simulations of reactor thermal hydraulics. To achieve the overall scalability of the parallel implementation, each component of the model needs to have the algorithmic scalability and the implementation scalability. Here, we mainly focus on the algorithmic scalability, since implementation scalability can be realized by utilizing and reengineering the existing software libraries for PDE simulations $[15,92,97]$ and Solvers $[14,51,75]$. In fact, the algorithmic scalabilities of the preconditioned Krylov solvers can also be achieved, as long as the preconditioners are effective.

In Chapter 9, we discuss the strategy of building scalable components for each physical process, namely parallel preconditioners and solvers for discrete diffusion, discrete convection-diffusion, and discrete Navier-Stokes equations. In Chapter 10, we demonstrate a case in which careful designed 


\section{CHAPTER 1. INTRODUCTION}

precondtioners lead to scalable performance for multiphase turbulence flows in reactor thermal hydraulics. In Chapter 11, we outline a research plan for parallel and scalable solutions to Dirichlet boundary control of CHT processes. 
Part I

\section{Problem Formulation and Numerical Building Blocks}




\section{Chapter 2}

\section{Problem Formulation}

\subsection{Overview}

We formulate the Dirichlet velocity boundary control problem for conjugate heat transfer processes in this chapter. Our assumption is that the fluid flow is steady, the thermal transport in the fluid-solid system is forced convection, and the buoyancy effect of the fluid is negligible. The mathematical formulation of the optimization problem consists of three parts:

- The objective function: the sum of the mismatch between the temperature in the fluid system and a prescribed temperature and the cost of the control.

- The constraints on the fluid velocity, pressure, and the boundary control: the incompressible Navier-Stokes equations of fluid motion.

- The constraints on the fluid velocity and the fluid-solid temperature: the diffusion equation of internal energy in the solid region and the convection-diffusion equation of internal energy in the fluid region.

We set up the state equations in Section 2.2 and the objective function in Section 2.3 


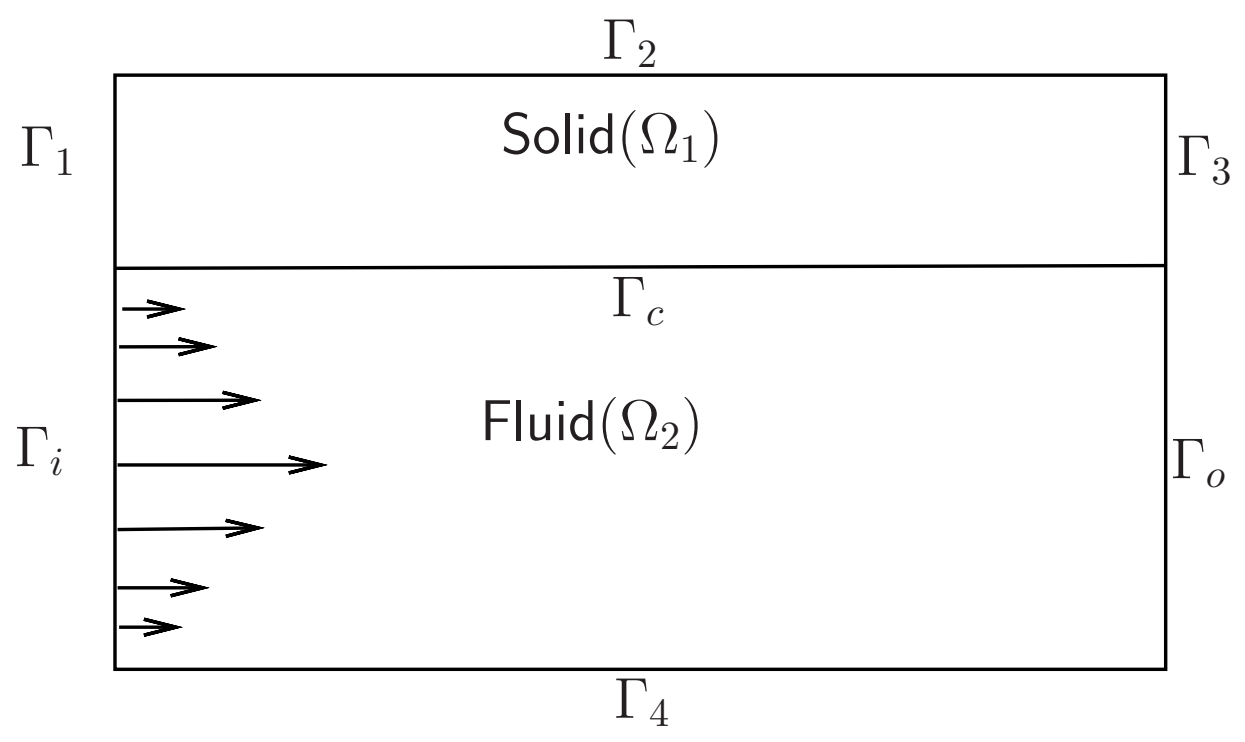

Figure 2.1: Physical domain of the coupled fluid-solid conjugate heat transfer problem.

\subsection{State Equations}

We refer to Figure 2.1 for a description of an idealized domain of the coupled fluid-solid system, in which the solid domain $\Omega_{1}$ represents a simplified fuel-gap-clad system in $2 D$ and the fluid domain $\Omega_{2}$ represents a coolant channel [100]. In the solid region $\Omega_{1}$, heat is generated by a volumetric heating source. Heat flux enters the fluid region $\Omega_{2}$ at the fluid-solid interface $\Gamma_{c}$. In $\Omega_{2}$, we have assumed that the thermal transport is driven by forced convection and the buoyancy effect can be neglected. Therefore, the fluid internal energy equation is decoupled from the fluid mass and momentum equations, and the fluid temperature is governed by the convection-diffusion equation, in which the convective "wind" is the fluid velocity field.

In $\Omega_{2}$, we further assume that the fluid density is constant and the dynamic viscosity is independent of the fluid temperature; therefore, we employ the steady equations for the conservation of mass and momentum with the constitutive stress relation of Newtonian fluids in the incompressible limit. Denoting by $\Gamma_{i}$ the inflow boundary, $\Gamma_{o}$ the outlet flow boundary, and $\Gamma_{c}$ and $\Gamma_{4}$ the no-slip 
boundaries, we write the incompressible Navier-Stokes equations of fluid motion $[60,66]$

$$
\text { (Fluid) }\left\{\begin{array}{rllll}
-v \nabla \cdot\left(\nabla \mathbf{u}+\nabla \mathbf{u}^{T}\right)+(\mathbf{u} \cdot \nabla) \mathbf{u}+\nabla p & = & \mathbf{b} & \text { in } & \Omega_{2} \quad \text { (Momentum Eq.) } \\
\nabla \cdot \mathbf{u} & = & 0 & \text { in } & \Omega_{2} \quad \text { (Mass Eq.) } \\
\mathbf{u} & = & 0 & \text { on } \quad \Gamma_{4} \text { and } \Gamma_{c} \\
\mathbf{u} & = & \mathbf{g} & \text { on } & \Gamma_{i} \\
\mathbf{n} \cdot\left(-p \mathbf{I}+v\left(\nabla \mathbf{u}+\nabla \mathbf{u}^{T}\right)\right) & = & 0 & \text { on } & \Gamma_{o},
\end{array}\right.
$$

where $\mathbf{u}=\mathbf{u}(x, y)$ is the velocity, $p$ is the kinematic pressure, $v=\mu / \rho$ is the kinematic viscosity, $\mathbf{b}$ is the body force and $\mathbf{g}$ is the Dirichlet boundary control profile at the inflow boundary. In this study, we assume the body force $\mathbf{b} \equiv 0$, and with

(The strain tensor) $\quad \varepsilon(\mathbf{u}):=\left(\nabla \mathbf{u}+\nabla \mathbf{u}^{T}\right) / 2$

(The stress tensor) $\quad \sigma(\mathbf{u}, p):=-p \mathbf{I}+2 v \varepsilon(\mathbf{u})$,

we have imposed the stress-free condition at the outlet boundary $\Gamma_{o}$.

For the temperature system in the fluid-solid region, $\Gamma_{o}, \Gamma_{1}, \Gamma_{2}, \Gamma_{3}$ and $\Gamma_{4}$ in Figure 2.1 are five adiabatic walls, and $\Gamma_{c}$ is the fluid-solid thermal interface, at which the convection heat transfer occurs between the solid and the fluid. On the inflow boundary $\Gamma_{i}$, we prescribe the fluid temperature. In what follows, we use the subscript $s$ to denote quantities in solid and $f$ to denote quantities in fluid. Neglecting the buoyancy effect, the fluid internal energy equation is

$$
\text { (Fluid) }\left\{\begin{array}{rllll}
-k_{f} \Delta T_{f}+\mathbf{u} \cdot \nabla T_{f} & = & 0 & \text { in } & \Omega_{2} \\
T_{f} & = & T_{s} & \text { on } & \Gamma_{c} \\
\mathbf{n} \cdot\left(k_{f} \nabla T_{f}-k_{s} \nabla T_{s}\right) & = & 0 & \text { on } & \Gamma_{c} \\
\mathbf{n} \cdot \nabla T & = & 0 & \text { on } & \Gamma_{4} \text { and } \Gamma_{o} \\
T_{f} & = & T_{0} & \text { on } & \Gamma_{i} .
\end{array}\right.
$$

Here, $T_{f}=T_{f}(x, y)$ is the fluid temperature, and $k_{f}$ is the thermal diffusivity of the fluid. The two boundary conditions posed on the fluid-solid interface $\Gamma_{c}$ are the continuity of temperature and heat flux. $T_{0}$ is the prescribed temperature profile at inflow and $\mathbf{u}$ is the velocity in the fluid motion equations (2.1). 
Denoting by $T_{s}=T_{s}(x, y)$ the solid temperature, $k_{s}$ the thermal diffusivity of the solid, and $q$ the volumetric heating source of unit $J /\left(s \cdot m^{3}\right)$, we write the solid internal energy equation

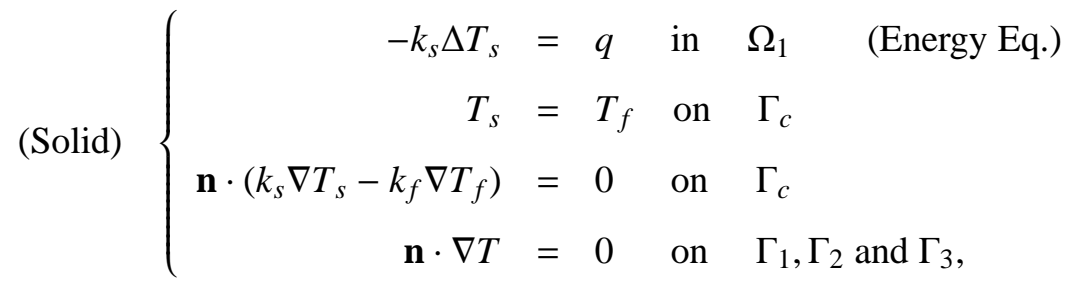

where we have posed the same continuity conditions as in (2.2) on $\Gamma_{c}$.

Whether to use velocity or temperature as the actuator for the boundary control of conjugate heat transfer processes makes a great difference in the coupling of the fluid velocity with the system temperature. In literature $[63,116]$, the case that temperature is considered as the boundary control device for conjugate heat transfer processes has been thoroughly discussed. There, the fluid velocity is merely a constant "wind" in the convection-diffusion equations of the fluid-solid internal energy. In contrast, in our case, as the control device is the inflow velocity profile, the fluid velocity field is strongly coupled with the temperature distribution in the optimization problem. In other words, each time we update the inlet velocity towards the optimal profile, we have to recalculate the entire fluid velocity field and then solve the internal energy equations for the fluid-solid temperature distribution.

\subsection{Objective Function}

To ensure the well-posedness of the Navier-Stokes equations in function spaces, the control profile g needs to be in $\mathbf{H}^{1 / 2}\left(\Gamma_{i}\right)$ [58], which is defined in Section 3.1. Therefore, it is safe to assume $\mathbf{g} \in \mathbf{H}^{1}\left(\Gamma_{i}\right)$, where $\mathbf{H}^{1}\left(\Gamma_{i}\right) \subset \mathbf{H}^{1 / 2}\left(\Gamma_{i}\right)$. In this study, we have chosen the following objective function to measure the system performance under a specific velocity control profile $\mathbf{g}$

$$
\underset{\left(\mathbf{u}, p, T_{f}, T_{s}, \mathbf{g}\right)}{\operatorname{minimize}} \mathcal{J}\left(\mathbf{u}, p, T_{f}, T_{s}, \mathbf{g}\right):=\frac{1}{2} \int_{\Omega_{d}}\left(T-T_{d}\right)^{2} d \mathbf{x}+\frac{\beta_{1}}{2} \int_{\Gamma_{i}}|\mathbf{g}|^{2} d s+\frac{\beta_{2}}{2} \int_{\Gamma_{i}}|\nabla \mathbf{g}|^{2} d s .
$$

Here, $T_{d}$ is the target temperature profile. See Section 7.2 for details on the target temperature $T_{d}$. $\Omega_{d} \in \Omega_{2}$ is the observation domain on which the temperature mismatch $\left(T-T_{d}\right)$ is measured. We 
note that $\Omega_{d}$ can be either the interfacial boundary $\Gamma_{c}$ or the whole fluid domain $\Omega_{2}$. The positive regularization parameters $\beta_{1}, \beta_{2}>0$ penalize the velocity control profile and its gradient. Their main function is to make the objective function convex, prevent infinitely large control and most importantly, ensure a smooth boundary control profile. See also the work by Gunzburger, et al. [65] for a similar formulation of the boundary control of Navier-Stokes equations. In [86], a regularization term using the $\mathbf{H}^{1 / 2}$ semi-norm of $\mathbf{g}$, based on the Steklov-Poincare operator, provides success in solving a blood-flow application of Dirichlet boundary control of Navier-Stokes equations. In [70], a regularization term using the $\mathbf{H}^{1}$ norm of $\mathbf{g}$ is successful in solving a flow matching problem in a two-dimensional cavity. We emphasize that a sufficiently large $\beta_{2}$ is essential for ensuring the boundedness of control $\mathbf{g}$ in $\mathbf{H}^{1}\left(\Gamma_{i}\right)$ and the existence of solutions to the Dirichlet boundary control problem (2.1)-(2.4) in infinite dimensional function spaces. We refer to Section 5.3 the discussion on the necessity of $\beta_{2}>0$ on the discrete level and its relation to the smoothing operator that resolves the nonsmoothness issue of the boundary control. 


\section{Chapter 3}

\section{Building Blocks for CHT simulations}

\subsection{Sobolev Spaces}

It is evident from equations (2.1)-(2.3) that in the CHT process, the fluid temperature is coupled with the solid temperature and the fluid velocity field. Therefore, The finite element modeling (FEM) of the CHT process involves diffusion, convection-diffusion, and incompressible Navier-Stokes equations. In Section 3.2, we discuss the FEM of diffusion and convection-diffusion equations. In Section 3.3, we discuss the FEM of incompressible Navier-Stokes equations. The FEM of CHT processes is discussed in Section 3.4

First, we define the appropriate Sobolev spaces for deriving the weak formulation of various equations. We note that mathematical symbols that are in bold face represent vector quantities. Introducing the Sobolev spaces on a bounded domain $\Omega \subset \mathbb{R}^{d}$, we denote by $L^{2}(\Omega)$ the space of square integrable scalar valued functions and by $L_{0}^{2}(\Omega)$ the space of square integrable scalar valued functions with zero integral mean at the Neumann boundary associated with stress-free condition. Moreover, we denote by $H^{1}(\Omega)$ the space of scalar-valued functions with square integrable first order derivatives and by $\mathbf{H}^{1}(\Omega)$ the product space $H^{1}(\Omega)^{d}$, the space of vector-valued functions with square integrable first order derivatives in $\Omega^{d}$. Assuming that $\Gamma \subset \Omega$ is a Lipschitz-continous boundary of the bounded domain $\Omega$ and the vector field $\mathbf{u}$ belongs to $\mathbf{H}^{1}(\Omega)^{d}$, then, by the Sobolev Embedding theorem, $\left.\mathbf{u}\right|_{\Gamma}$, the trace of $\mathbf{u}$, belongs to $\mathbf{H}^{1 / 2}(\Gamma)^{d-1}$. In fact, the spatial dimension is 
clear from the notation of $\Omega$ and $\Gamma$. Therefore, we write $\mathbf{u} \in \mathbf{H}^{1}(\Omega)$ and $\left.\mathbf{u}\right|_{\Gamma} \in \mathbf{H}^{1 / 2}(\Gamma)$ without the dimensionality $d$ and $d-1$. Furthermore, we denote by $\mathbf{H}^{-k}(\Omega)$ with $k>0$ the dual space of $\mathbf{H}^{k}(\Omega)$. Members in $\mathbf{H}^{-k}(\Omega)$ are generalized functions in the sense of distributions.

\subsection{Diffusion and Convection-diffusion Equations}

\subsubsection{Diffusion equations}

The heat conduction for a homogeneous and isotropic material is governed by the diffusion equation

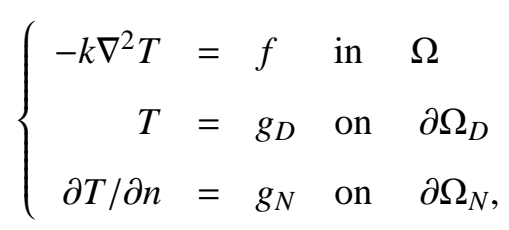

where $k$ is the diffusion coefficient. We denote the boundary of the domain as $\partial \Omega$ with $\partial \Omega=\partial \Omega_{D} U$ $\partial \Omega_{N}$. And we impose Dirichlet and Neumann boundary condition on $\Omega_{D}$ and $\Omega_{N}$, respectively. Using the Lax-Milgram theorem [31], we can show that $T$ belongs to $H^{1}(\Omega)$, where

$$
H^{1}(\Omega)=\left\{T \in H^{1} \mid T=g_{D} \text { on } \partial \Omega_{D}\right\}
$$

Moreover, we introduce the test function $v \in H_{0}^{1}(\Omega)$, where

$$
H_{0}^{1}(\Omega)=\left\{v \in H^{1}(\Omega) \mid v=0 \text { on } \partial \Omega_{D}\right\}
$$

The weak formulation of (3.1) is to find such $T \in H^{1}(\Omega)$ that

$$
k \int_{\Omega} \nabla T \cdot \nabla v=\int_{\Omega} f v+\int_{\partial \Omega_{N}} k g_{N} v \text { for all } v \in H_{0}^{1}(\Omega) .
$$

Next, we introduce the finite dimensional space $S_{0}^{h} \subset H_{0}^{1}(\Omega)$ of test functions with basis $\left\{\phi_{1}, \phi_{2}, \ldots \phi_{n}\right\}$ and space $S^{h} \subset H^{1}(\Omega)$ of trial functions with basis $\left\{\phi_{1}, \phi_{2}, \ldots \phi_{n}, \phi_{n+1}, \ldots, \phi_{n+n_{D}}\right\}$, where $n_{D}$ is the number of nodes on the Dirichlet boundary and $n$ is the number of all element nodes except those on the Dirichlet boundary. The finite element approximation of the temperature

$$
T^{h}=\sum_{j=1}^{n} \phi_{j} T_{j}^{h}+\sum_{j=n+1}^{n_{D}} \phi_{j} T_{j}^{h}
$$


can be uniquely represented as a vector $\mathbf{T}=\left(T_{1}, T_{2}, \ldots, T_{n}\right)^{T}$ with the boundary data $T_{j}, j=n+$ $1, \ldots, n+n_{D}$ known. Finally, we take the the test function $v=\phi_{i}$ and apply it to the weak formulation (3.1) with $T=T_{h}$, we obtain a linear algebraic system for the temperature approximation $\mathbf{T}=$ $\left(T_{1}, T_{2}, \ldots, T_{n}\right)^{T}$

$$
k \sum_{j=1}^{n} T_{j} \int_{\Omega} \nabla \phi_{j} \cdot \nabla \phi_{i}=\int_{\Omega} f \phi_{i}+\int_{\partial \Omega_{N}} k g_{N} \phi_{i}-k \sum_{j=n+1}^{n_{D}} T_{j} \int_{\Omega} \nabla \phi_{j} \cdot \nabla \phi_{i},
$$

for $i=1,2, \ldots n$. In matrix form, we can write the linear system as

$$
A \mathbf{T}=\mathbf{f},
$$

where the symmetric matrix

$$
A=\left[a_{i j}\right], a_{i j}=k \int_{\Omega} \nabla \phi_{j} \cdot \nabla \phi_{i}=k \int_{\Omega} \nabla \phi_{i} \cdot \nabla \phi_{j}
$$

and the right-hand side forcing term $\mathbf{f}=\left[f_{i}\right]$. We display the finite element solution of the diffusion equation on a square domain with $f(x) \equiv 10$ in Figure 3.1.

\subsubsection{Convection-diffusion Equations}

The convection-diffusion equation describes the distribution of a quantity, such as temperature, density or concentration, inside a flowing medium which has a prescribed velocity field (wind) $\mathbf{w}=\left(w_{x}, w_{y}\right)^{T}$. When the quantity of interest is the fluid temperature, we write the steady-state energy equation of the fluid as

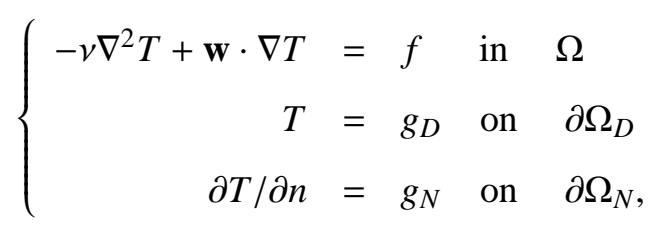

where $v$ is the diffusion coefficient. We denote the boundary of the domain as $\partial \Omega$ with $\partial \Omega=\partial \Omega_{D} \cup$ $\partial \Omega_{N}$, and we impose Dirichlet and Neumann boundary condition on $\partial \Omega_{D}$ and $\partial \Omega_{N}$, respectively. We assume that we are dealing with pipe flow. We denote $\partial \Omega_{D}$ as the inflow boundary and $\partial \Omega_{N}$ as the outflow and wall boundary. We impose the homogeneous Neumann boundary condition at the outlet. Using the Lax-Milgram theorem, we can show that $T$ has the regularity in $H^{1}(\Omega)$, where

$$
H^{1}(\Omega)=\left\{T \in H^{1} \mid T=g_{D} \text { on } \partial \Omega_{D}\right\}
$$




\section{FEM solution contour plot}

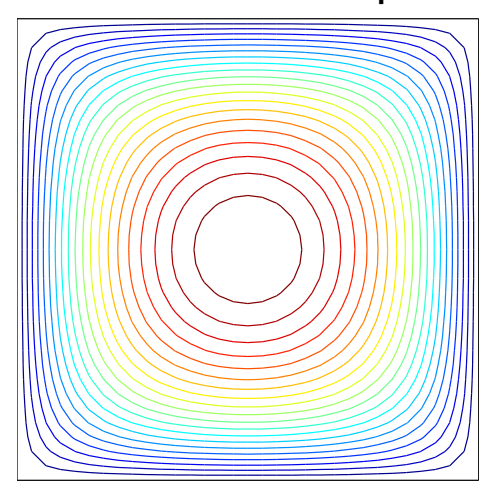

FEM solution mesh plot

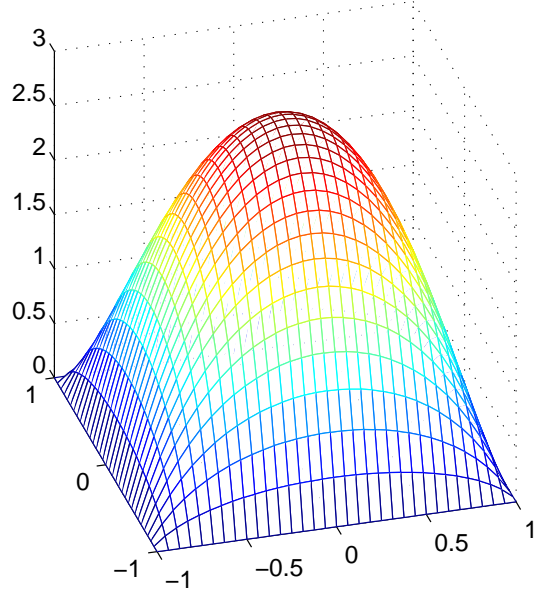

Figure 3.1: Diffusion equation on a square domain with constant forcing. 
Moreover, We introduce the test function $\phi \in H_{0}^{1}(\Omega)$, where

$$
H_{0}^{1}(\Omega)=\left\{\phi \in H^{1}(\Omega) \mid \phi=0 \text { on } \partial \Omega_{D}\right\}
$$

The weak formulation of (3.5) is to find $T \in H^{1}(\Omega)$ such that

$$
v \int_{\Omega} \nabla T \cdot \nabla \phi+\int_{\Omega}(\mathbf{w} \cdot \nabla T) \phi=\int_{\Omega} f \phi+\int_{\partial \Omega_{N}} v g_{N} \phi \quad \text { for all } \phi \in H_{0}^{1}(\Omega) .
$$

This is the standard Galerkin formulation. However, the numerical solution of the temperature $T$ based on this formulation can be inaccurate when the mesh is not fine enough to resolve all the details close to the boundary layer. An under-resolved boundary layer, in which a large second derivative of $T$ cannot be represented, may cause oscillation in the numerical approximation, which can further pollute the solution in the interior region. A popular remedy for this issue is to use the Stream Upwinding/Petrov Galerkin (SUPG) [80] [48] formulation for the weak formulation

$$
v \int_{\Omega} \nabla T \cdot \nabla \phi+\int_{\Omega}(\mathbf{w} \cdot \nabla T) \phi+s \int_{\Omega}(\mathbf{w} \cdot \nabla T)(\mathbf{w} \cdot \nabla \phi)-s v \int_{\Omega}\left(\nabla^{2} T\right)(\mathbf{w} \cdot \nabla \phi)=l(\phi),
$$

where

$$
l(\phi)=\int_{\Omega} f \phi+\int_{\partial \Omega_{N}} v g_{N} \phi+s \int_{\Omega} f \mathbf{w} \cdot \nabla \phi .
$$

Comparing to (3.6), the extra diffusion terms introduced in (3.7) stabilize the numerical scheme and suppress the numerical oscillation. A good choice of the streamline-diffusion parameter $s$ is given by $[48,52]$

$$
s= \begin{cases}\frac{h}{2|\mathbf{w}|}\left(1-\frac{1}{P_{h}}\right) & \text { if } P_{h}>1 \\ 0 & \text { if } P_{h} \leq 1 .\end{cases}
$$

$P_{h}:=|\mathbf{w} h| /(2 v)$ is the mesh Peclet number denoting the ratio of the convection over diffusion inside a mesh cell with size $h$. Next, we introduce the finite dimensional space $S_{0}^{h} \subset H_{0}^{1}(\Omega)$ of test functions with basis $\left\{\phi_{1}, \phi_{2}, \ldots \phi_{n}\right\}$ and space $S^{h} \subset H^{1}(\Omega)$ of trial functions with basis $\left\{\phi_{1}, \phi_{2}, \ldots \phi_{n}, \phi_{n+1}, \ldots, \phi_{n+n_{D}}\right\}$, where $n_{D}$ is the number of nodes on the Dirichlet boundary and $n$ is the number of all element nodes except those on the Dirichlet boundary. The finite element approximation of the temperature

$$
T^{h}=\sum_{j=1}^{n} \phi_{j} T_{j}^{h}+\sum_{j=n+1}^{n_{D}} \phi_{j} T_{j}^{h}
$$


can be uniquely represented as a vector $\mathbf{T}=\left(T_{1}, T_{2}, \ldots, T_{n}\right)^{T}$ with the boundary data $T_{j}, j \geq n+1$ known. Finally, we take the the test function $\phi=\phi_{i}$ and apply it to the weak formulation (3.8) with $T=T_{h}$. For the simplicity of notation, we take the linear basis functions, homogenous source function $f$ and Neumann boundary condition $g_{N}=0$, we obtain a linear algebraic system for the temperature approximation $\mathbf{T}=\left(T_{1}, T_{2}, \ldots, T_{n}\right)^{T}$

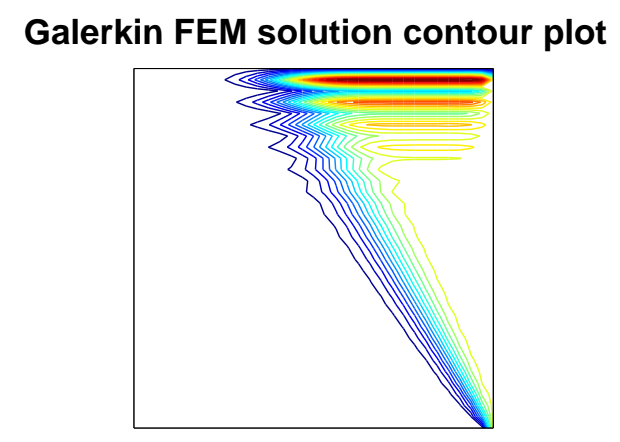

SUPG solution contour plot

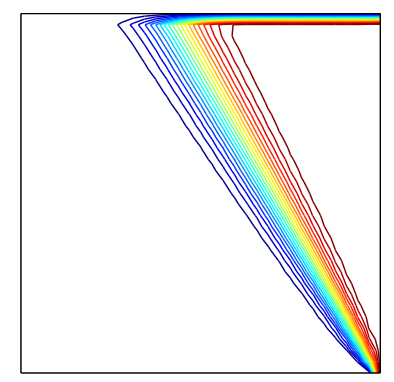

\section{Galerkin FEM solution mesh plot}

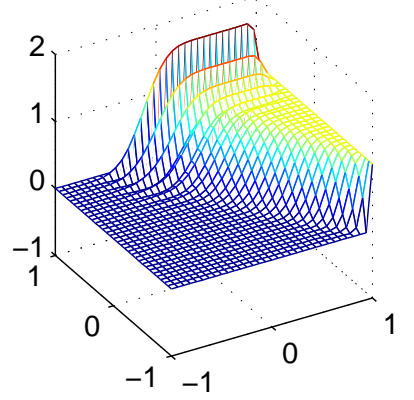

SUPG solution mesh plot

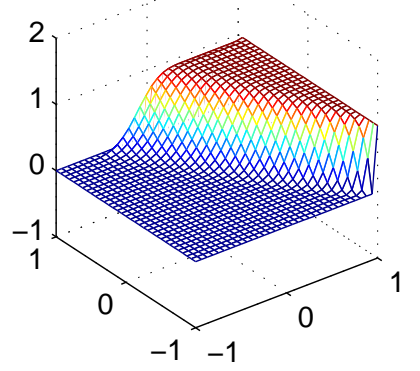

Figure 3.2: Convection-diffusion equation on a square domain with a constant wind blowing skew to the grid. Comparison of Galerkin FEM and SUPG FEM.

$$
\begin{aligned}
& v \sum_{j=1}^{n+n_{D}} T_{j} \int_{\Omega} \nabla \phi_{j} \cdot \nabla \phi_{i}+\sum_{j=1}^{n+n_{D}} T_{j} \int_{\Omega}\left(\mathbf{w} \cdot \nabla \phi_{j}\right) \phi_{i}+s \sum_{j=1}^{n+n_{D}} T_{j} \int_{\Omega}\left(\mathbf{w} \cdot \nabla \phi_{j}\right)\left(\mathbf{w} \cdot \nabla \phi_{i}\right)=0 \\
& v \sum_{j=1}^{n} T_{j} \int_{\Omega} \nabla \phi_{j} \cdot \nabla \phi_{i}+\sum_{j=1}^{n} T_{j} \int_{\Omega}\left(\mathbf{w} \cdot \nabla \phi_{j}\right) \phi_{i}+s \sum_{j=1}^{n} T_{j} \int_{\Omega}\left(\mathbf{w} \cdot \nabla \phi_{j}\right)\left(\mathbf{w} \cdot \nabla \phi_{i}\right)=g_{h}\left(\phi_{i}\right),
\end{aligned}
$$


where

$$
g_{h}\left(\phi_{i}\right)=-v \sum_{j=n+1}^{n_{D}} T_{j} \int_{\Omega} \nabla \phi_{j} \cdot \nabla \phi_{i}-\sum_{j=n+1}^{n_{D}} T_{j} \int_{\Omega}\left(\mathbf{w} \cdot \nabla \phi_{j}\right) \phi_{i}-s \sum_{j=n+1}^{n_{D}} T_{j} \int_{\Omega}\left(\mathbf{w} \cdot \nabla \phi_{j}\right)\left(\mathbf{w} \cdot \nabla \phi_{i}\right) .
$$

In matrix form, we can write the linear system as

$$
F \mathbf{T}=\mathbf{g},
$$

where

$$
\begin{aligned}
& F=v A+N+S ; \\
& A=\left[a_{i j}\right], a_{i j}=\int_{\Omega} \nabla \phi_{j} \cdot \nabla \phi_{i} ; \\
& N=\left[n_{i j}\right], n_{i j}=\int_{\Omega}\left(\mathbf{w} \cdot \nabla \phi_{j}\right) \phi_{i} ; \\
& S=\left[s_{i j}\right], s_{i j}=s \int_{\Omega}\left(\mathbf{w} \cdot \nabla \phi_{j}\right)\left(\mathbf{w} \cdot \nabla \phi_{i}\right) ; \\
& \mathbf{g}=\left[g_{i}\right], g_{i}=g_{h}\left(\phi_{i}\right) .
\end{aligned}
$$

We display in Figure 3.2 the finite element solutions of a modified test case from [31], namely the convection-diffusion equation on a square domain with a constant wind blowing from southeast at $30^{\circ}$ angle.

\subsection{Incompressible Navier-Stokes Equations}

We write the steady-state incompressible viscous Navier-Stokes equation

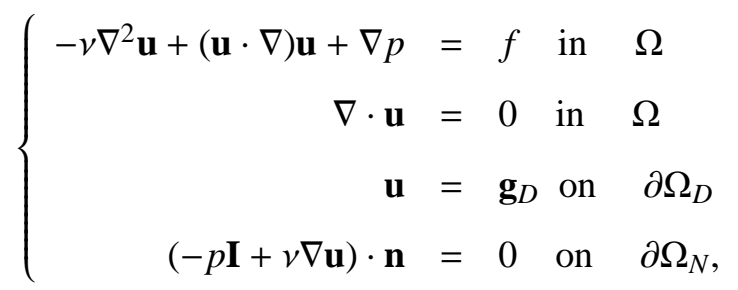

where $v$ is the kinematic viscosity of the fluid, $\partial \Omega_{D}$ is the inflow and wall boundary, and $\partial \Omega_{N}$ is the outflow boundary. We note that the first equation in (3.13) is equivalent to that of (2.1). The velocity field $\mathbf{u}$ and the pressure $p$ are characterized by the non-dimensional Reynolds number $R e:=U L / v$, 
where $L$ is the characteristic length of the domain. Introducing the trial and test functional spaces for the velocity $\mathbf{u}$

$$
\begin{aligned}
& \mathbf{H}^{1}(\Omega)=\left\{\mathbf{v} \in \mathbf{H}^{1} \mid \mathbf{v}=\mathbf{g}_{D} \text { on } \partial \Omega_{D}\right\} \\
& \mathbf{H}_{0}^{1}(\Omega)=\left\{\mathbf{v} \in \mathbf{H}^{1} \mid \mathbf{v}=0 \text { on } \partial \Omega_{D}\right\},
\end{aligned}
$$

and the right space for the pressure $p, L_{0}^{2}(\Omega)$ [58], we derive the weak formulation of (3.13), which is to find $\mathbf{u} \in \mathbf{H}^{1}(\Omega)$ and $p \in L_{0}^{2}(\Omega)$ such that

$$
\left\{\begin{aligned}
v \int_{\Omega} \nabla \mathbf{u}: \nabla \mathbf{v}+\int_{\Omega}(\mathbf{u} \cdot \nabla \mathbf{u}) \cdot \mathbf{v}-\int_{\Omega} p(\nabla \cdot \mathbf{v}) & =\int_{\Omega} f \cdot \mathbf{v} & & \text { for all } \mathbf{v} \in \mathbf{H}_{0}^{1}(\Omega) \\
-\int_{\Omega} q(\nabla \cdot \mathbf{u}) & =0 & & \text { for all } q \in L^{2}(\Omega),
\end{aligned}\right.
$$

where $\nabla \mathbf{u}: \nabla \mathbf{v}=\mathbf{u}_{i, j} \mathbf{v}_{i, j}$ in index notation. The difficulty of solving the Navier-Stokes equations is mainly caused by the nonlinear term inside (3.15)

$$
c(\mathbf{a} ; \mathbf{b}, \mathbf{c})=\int_{\Omega}(\mathbf{a} \cdot \nabla \mathbf{b}) \cdot \mathbf{c},
$$

which is a trilinear form defined on the product space $\mathbf{H}^{1}(\Omega) \times \mathbf{H}^{1}(\Omega) \times \mathbf{H}_{0}^{1}(\Omega)$. In order to solve the nonlinear system (3.15), we linearize the nonlinear term and use iterative scheme [48]. Given iterate $\left(\mathbf{u}^{k}, p^{k}\right)$, we set

$$
\begin{aligned}
& \mathbf{u}^{k+1}=\mathbf{u}^{k}+\delta \mathbf{u} \\
& p^{k+1}=p^{k}+\delta p
\end{aligned}
$$

and enforce that $\left(\mathbf{u}^{k+1}, p^{k+1}\right)$ satisfies the weak formulation (3.15). Then, neglecting the second order term in $\delta \mathbf{u}$, we get an equation system for the update $(\delta \mathbf{u}, \delta p)$

$$
\left\{\begin{aligned}
c\left(\delta \mathbf{u} ; \mathbf{u}^{k}, \mathbf{v}\right)+c\left(\mathbf{u}^{k} ; \delta \mathbf{u}, \mathbf{v}\right)+v \int_{\Omega} \nabla \delta \mathbf{u}: \nabla \mathbf{v}-\int_{\Omega} \delta p(\nabla \cdot \mathbf{v}) & =R^{k}(\mathbf{v}) \\
-\int_{\Omega} q(\nabla \cdot \delta \mathbf{u}) & =r^{k}(q),
\end{aligned}\right.
$$

with right-hand side terms

$$
\begin{aligned}
& R^{k}(\mathbf{v})=\int_{\Omega} f \cdot \mathbf{v}-c\left(\mathbf{u}^{k} ; \mathbf{u}^{k}, \mathbf{v}\right)-v \int_{\Omega} \nabla \mathbf{u}^{k}: \nabla \mathbf{v}+\int_{\Omega} p^{k}(\nabla \cdot \mathbf{v}) \\
& r^{k}(q)=\int_{\Omega} q\left(\nabla \cdot \mathbf{u}^{k}\right) .
\end{aligned}
$$

The above system (3.17) is the Newton update system, and the resulting iterative scheme is Newton's method. We note that if we also neglect the term $c\left(\delta \mathbf{u} ; \mathbf{u}^{k}, \mathbf{v}\right)$ in (3.17), the resulting iterative scheme is Picard's method. 
We assume that the trial function space for the velocity approximation is $\mathbf{u}^{h} \in \mathbf{H}^{1}(\Omega)$ with basis functions $\left\{\boldsymbol{\phi}_{j}\right\}=\left\{\left[\begin{array}{c}\phi_{1} \\ 0\end{array}\right],\left[\begin{array}{c}\phi_{2} \\ 0\end{array}\right], \ldots,\left[\begin{array}{c}\phi_{n_{U}+n_{D}} \\ 0\end{array}\right],\left[\begin{array}{c}0 \\ \phi_{1}\end{array}\right], \ldots,\left[\begin{array}{c}0 \\ \phi_{n_{U}+n_{D}}\end{array}\right]\right\}$, and the trial functional space for the pressure approximation is $p^{h} \in L_{0}^{2}(\Omega)$ with basis functions $\left\{\psi_{j}\right\}$. Then, we can write

$$
\begin{aligned}
\mathbf{u}^{h} & =\sum_{j=1}^{n_{U}} u_{j} \boldsymbol{\phi}_{j}+\sum_{j=n_{U}+1}^{n_{U}+n_{D}} u_{j} \boldsymbol{\phi}_{j} ; \\
\delta \mathbf{u}^{h} & =\sum_{j=1}^{n_{U}} \delta u_{j} \boldsymbol{\phi}_{j} \\
p^{h} & =\sum_{j=1}^{n_{P}} p_{j} \psi_{j} \\
\delta p^{h} & =\sum_{j=1}^{n_{P}} \delta p_{j} \psi_{j}
\end{aligned}
$$

where $n_{D}$ is the degrees of freedom (DOF) of the velocity field at Dirichlet boundary, $n_{U}$ the rest of the DOF of the velocity field and $n_{P}$ the DOF of the pressure distribution. Plugging $\mathbf{u}^{h}$ and $p^{h}$ at the iteration $k$ into (3.17), we obtain the linear algebraic system of the Newton update $\left[\delta \mathbf{u}_{x}, \delta \mathbf{u}_{y}, \delta p\right]^{T}$

$$
\left(\begin{array}{ccc}
A+N+W_{x x} & W_{x y} & B_{x}^{T} \\
W_{y x} & A+N+W_{y y} & B_{y}^{T} \\
B_{x} & B_{y} & 0
\end{array}\right)\left(\begin{array}{c}
\delta \mathbf{u}_{x} \\
\delta \mathbf{u}_{y} \\
\delta p
\end{array}\right)=\left(\begin{array}{c}
\mathbf{R}_{x} \\
\mathbf{R}_{y} \\
\mathbf{r}
\end{array}\right),
$$

with

$$
\begin{aligned}
& A=\left[a_{i j}\right], a_{i j}=v \int_{\Omega} \nabla \phi_{j} \cdot \nabla \phi_{i} ; \\
& B_{\alpha}=\left[b_{\alpha i j}\right], b_{\alpha i j}=-\int_{\Omega} \psi_{i} \frac{\partial \phi_{j}}{\partial \alpha} ; \\
& N=\left[n_{i j}\right], n_{i j}=\int_{\Omega}\left(u_{x}^{h} \frac{\partial \phi_{j}}{\partial x}+u_{y}^{h} \frac{\partial \phi_{j}}{\partial y}\right) \phi_{i} ; \\
& W_{\alpha \beta}=\left[w_{\alpha \beta i j}\right], w_{\alpha \beta i j}=\int_{\Omega} \frac{\partial u_{\alpha}^{h}}{\partial \beta} \phi_{i} \phi_{j},
\end{aligned}
$$

where $\alpha, \beta \in\{x, y\}$. We display the Q2-Q1 stable Galerkin finite element solution of Poiseuille channel flow with parabolic inflow boundary condition $\left(1-y^{2}, 0\right)^{T}$ in Figure 3.3. 
CHAPTER 3. BUILDING BLOCKS FOR CHT SIMULATIONS
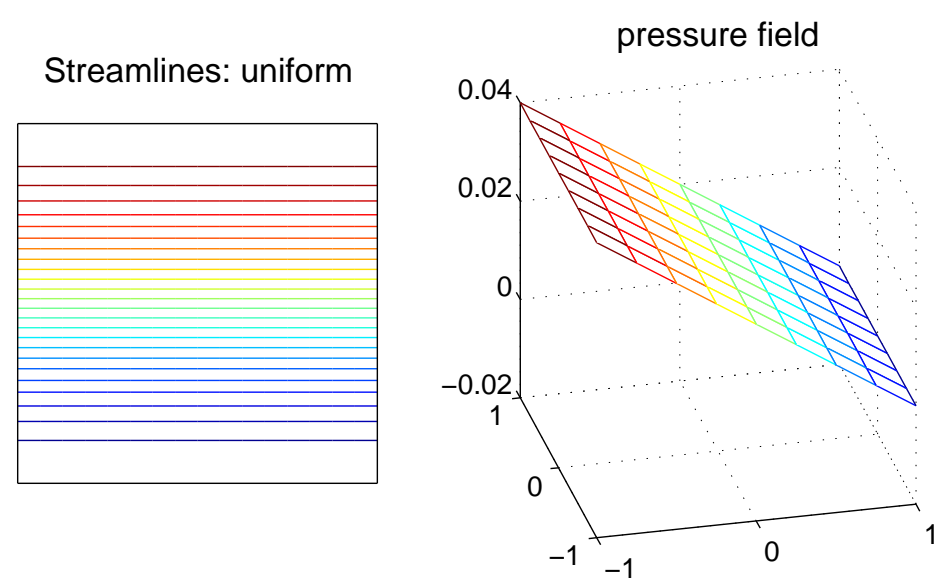

Figure 3.3: Q2-Q1 solution of Poiseuille channel flow with parabolic inflow boundary condition and $R e=100$. 


\subsection{Conjugate Heat Transfer Modeling and its Verification}

It is clear that the CHT model (2.1)-(2.3) involves all numerical building blocks discussed in Section 3.2 and Section 3.3. For the simplified geometry shown in Figure 2.1, we have used the Q2Q1 Taylor-Hood elements, namely piecewise-quadratic basis functions for the fluid velocity and piecewise-linear basis functions for the pressure, and Q2 elements, namely piecewise-quadratic basis functions for the fluid-solid temperature. This leads to an approximation that allows comparable accuracy between the velocity and temperature variables [46]. We display the mesh layout for the fluid-solid domain in Figure 3.4. The details about the discretization of the CHT state equations (2.1)-(2.3) are discussed in Chapter 5 .

$$
\text { Q2 velocity dofs Q1 pressure dofs Q2 temperature dofs }
$$
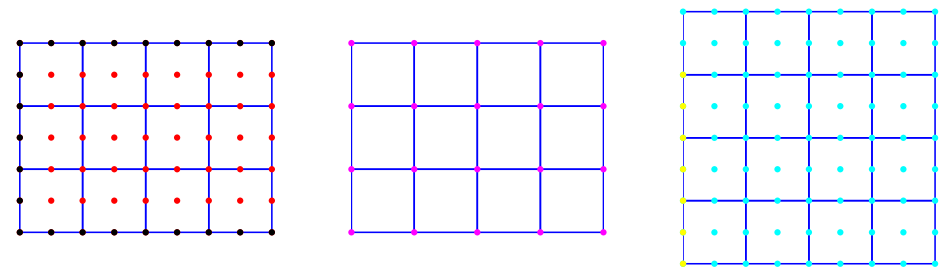

Figure 3.4: Q2-Q1-Q2 grid of the CHT problem domain.

\subsubsection{Model Verification in Multiphysics Simulations}

It is important to validate and verify the software after its implementation, since this is the only way to gain confidence that the code is solving the correct equations and solving them correctly. For simulations that involve multiphysics components, we have found that it is helpful to first verify each physics separately, and then to set up test cases to verify two or more physics jointly.

To demonstrate the verification process, we verify the part of the CHT code that solves the cou- 
pled diffusion (solid) and convection-diffusion (fluid) equations on the fluid-solid domain in Figure 2.1. Our assumption is that the velocity in the fluid region is given. For the coupled temperature equations (2.2) and (2.3), the FEM simulation of this system consists of five parts, namely, the discrete diffusion operators $A_{f}$ in the fluid region, $A_{s}$ in the solid region, the discrete convection operator $N_{f}$ in the fluid region, and the discrete source terms $q_{f}$ and $q_{s}$.

In the first test, we verify the diffusion operators $A_{f}$ and $A_{s}$ and the source terms $q_{f}$ and $q_{s}$. We consider Poisson's equation $-\Delta T=1$, which has an exact solution similar to Example 1.1.3 in [48]

$$
T(x, y)=\frac{2(1+y)}{(3+x)^{2}+(1+y)^{2}}+1 .
$$

The solution of this problem is posed on the same fluid-solid domain as shown in Figure 2.1. Although the thermal diffusivities in both subdomains are the same, we assemble the FEM components $A_{f}, A_{s}, q_{f}$ and $q_{s}$ in the fluid-solid domain separately by using the fluid equation (2.2) and solid equation (2.3), respectively. This is for testing purposes only. The grid is shown in Figure 3.5. The computed temperature and the exact termperature are shown in Figure 3.6 and Figure 3.7. The absolute difference between the computed solution of the CHT code and the analytical solution is $O\left(10^{-9}\right)$, and this verifies the FEM components $A_{f}, A_{s}, q_{f}$ and $q_{s}$ in the CHT forward simulation.

$$
\text { Q2 velocity dofs Q1 pressure dofs Q2 temperature dofs }
$$
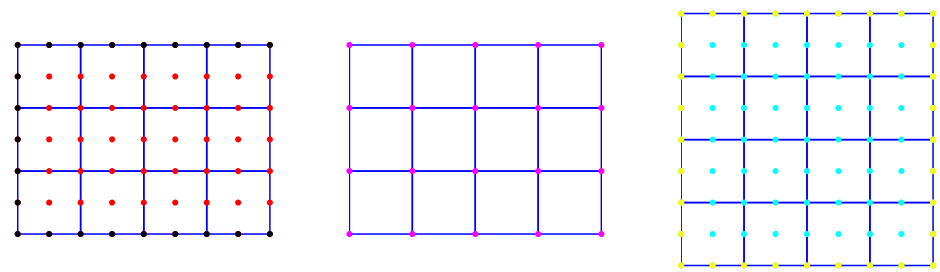

Figure 3.5: Q2-Q2 grid of Poisson's equation. 

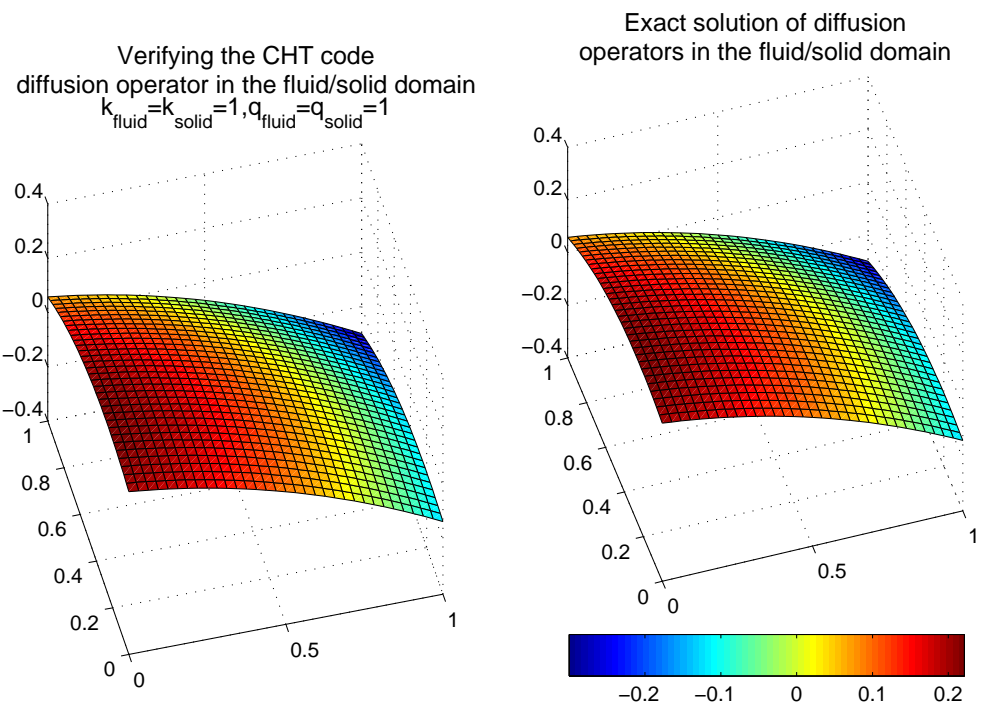

Figure 3.6: Simulation results for Poisson's equation.

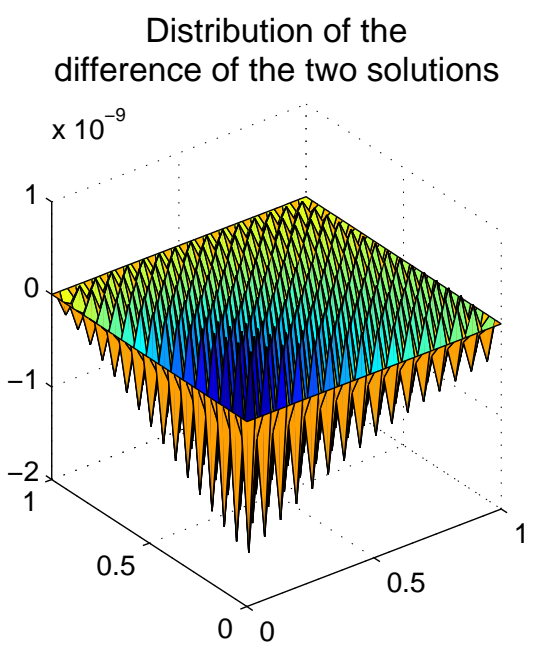

Figure 3.7: Error plot for Poisson's equation. 
In the second test, we verify the convection operator $N_{f}$ and diffusion operator $A_{f}$. We note that both $N_{f}$ and $A_{f}$ only appear in the fluid region; therefore, without loss of generality, we adjust the CHT domain in Figure 2.1 such that the fluid region is now the whole domain and $\Omega_{1}$ becomes $\emptyset$. In other words, the fluid and the fluid-solid domains in Figure 3.8 are both $[0,1] \times[0,1]$ now. We study a benchmark problem modified from the Example 3.1.2 in [48]. We set the thermal diffusivity as $1 / 200$ and the vertical wind $\mathbf{u}=\left(0,1+(x+1)^{2} / 4\right)$, and we apply Dirichlet boundary conditions on the bottom, left and right boundaries, namely,

$$
\left\{\begin{array}{l}
T=1 \quad \text { on } \quad y=0, \\
T=1-x^{3} \quad \text { on } \quad x=0 \\
T=1-x^{2} \text { on } \quad x=1 .
\end{array}\right.
$$

On the top boundary $y=1$, we impose the natural homogenous outflow boundary conditions. When the system is in steady state, there will be two characteristic layers on the left and right boundaries. In Figure 3.9, we compare the simulation result of the CHT code with that of the Q1 FEM code using Galerkin approximation with 16 macroelements. We show the difference of these two computed solutions in Figure 3.10. It is evident that the difference is $O\left(10^{-3}\right)$ in the interior of the domain and $O\left(10^{-2}\right)$ when it gets closer to the left and right boundary layer regions. This verifies the convection operator $N_{f}$ and diffusion operator $A_{f}$. Finally, we have verified all five components, $N_{f}, A_{f}, A_{s}, q_{f}$, and $q_{s}$, of the CHT simulation code. Therefore, we have the confidence that the fluid-solid internal energy equations are solved correctly. 

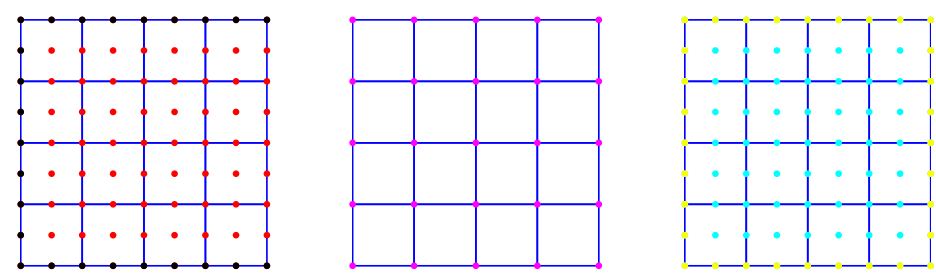

Figure 3.8: Q2-Q2 grid of the Convection-Diffusion test problem.
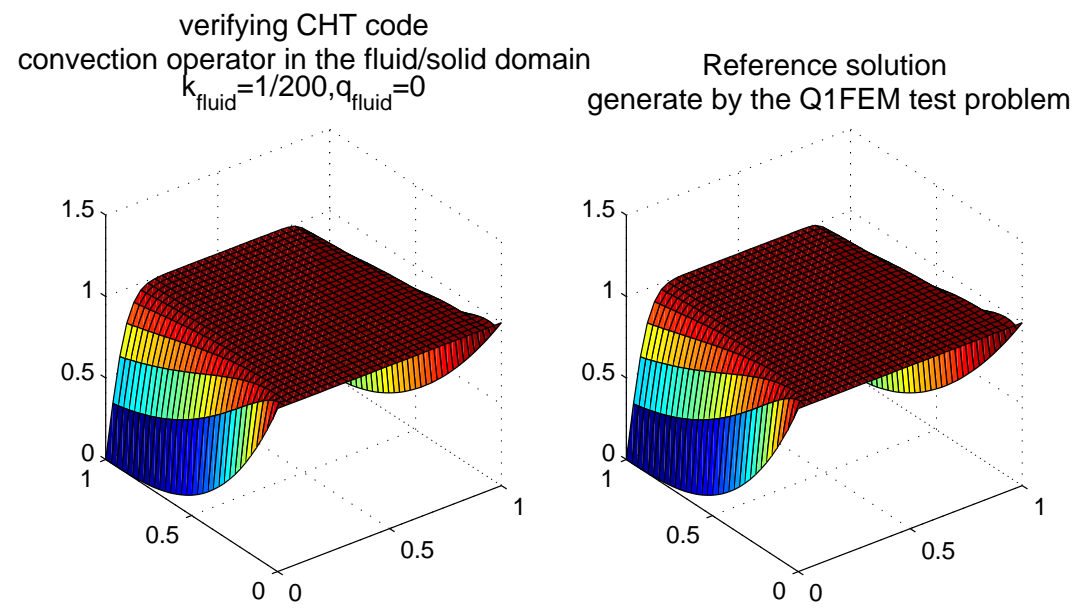

Figure 3.9: Comparison of simulation results from the CHT code and the Q1 Galerkin code for Convection-Diffusion test problem. 


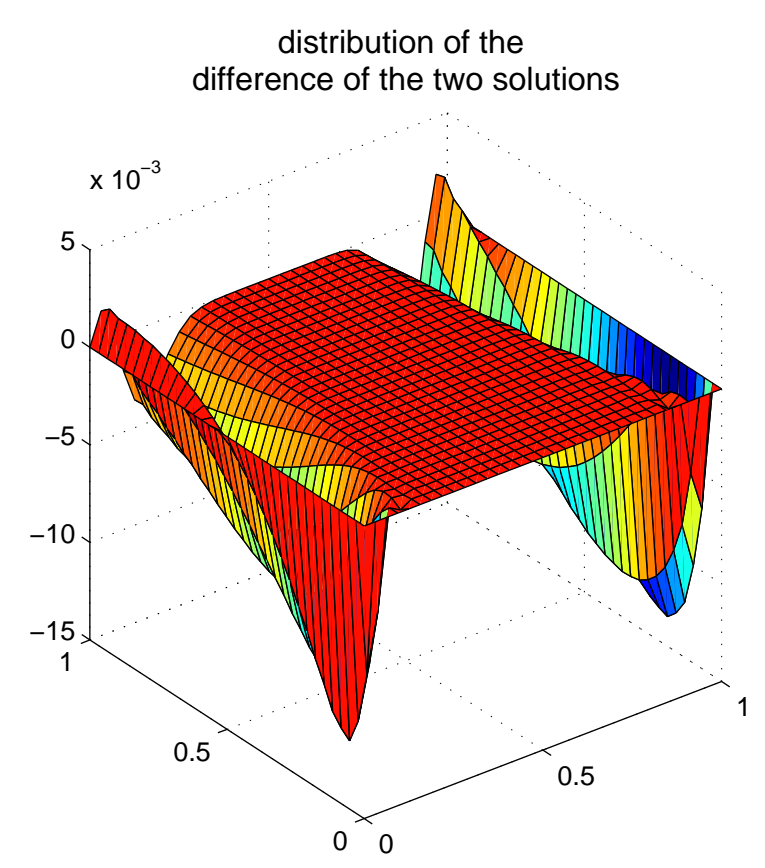

Figure 3.10: Difference of the two simulation results for the Convection-Diffusion test problem. 


\section{Part II}

\section{Smooth and Robust Solutions for}

\section{Dirichlet Boundary Control of}

\section{Fluid-Solid Conjugate Heat Transfer \\ Problems}




\section{Chapter 4}

\section{Optimality Conditions in Infinite}

\section{Dimensional Spaces}

\subsection{Overview}

To ensure the well-posedness of Navier-Stokes equations, we have assumed that the control data $\mathbf{g} \in \mathbf{H}^{1}\left(\Gamma_{i}\right)$ and chosen $\beta_{2}>0$. See Section 2.3. In fact, after deriving the optimality conditions of the Dirichlet velocity boundary control (DVBC) of the CHT process, we can assure that the assumption $\mathbf{g} \in \mathbf{H}^{1}\left(\Gamma_{i}\right)$ is valid. In Section 4.2, the variational form of the state equations is derived and the appropriate function spaces for solutions are determined. In Section 4.3, we derive the first order optimality conditions and the adjoint equations for the boundary control problem of the CHT process. Based on the derivation in Section $4.2-4.3$, Section 4.4 discusses the necessity of the regularization on the control gradient in the objective function for ensuring the well-posedness of the optimal control problem and the resulting regularity estimate on the boundary control profile. Features of the optimization codes are documented in the Appendix B. 


\subsection{Weak Form of the State Equations}

In this section, we derive the weak formulation of the state equations (2.1), (2.2), and (2.3) for the CHT process. We note that mathematical symbols that are in bold face represent vector quantities. Using the definition of the Sobolev spaces in Section 3.1, we define the trial and test function spaces on the fluid domain $\Omega_{2}$ for the velocity field $\mathbf{u}$ :

$$
\begin{aligned}
& \mathbf{H}^{1}\left(\Omega_{2}\right)=\left\{\mathbf{v} \in \mathbf{H}^{1}\left(\Omega_{2}\right) \mid \mathbf{v}=\mathbf{g}_{D} \text { on } \partial \Omega_{2 D}\right\} \\
& \mathbf{H}_{0}^{1}\left(\Omega_{2}\right)=\left\{\mathbf{v} \in \mathbf{H}^{1}\left(\Omega_{2}\right) \mid \mathbf{v}=0 \text { on } \partial \Omega_{2 D}\right\} .
\end{aligned}
$$

Here, $\partial \Omega_{2 D}=\Gamma_{c} \cup \Gamma_{4}$ in Figure 2.1 and $\mathbf{g}_{D}$ represents the Dirichlet velocity boundary conditions prescribed on $\partial \Omega_{2 D}$. The trial and test function spaces for the pressure $p$ are $L_{0}^{2}\left(\Omega_{2}\right)$ and $L^{2}\left(\Omega_{2}\right)$, respectively. The $L_{0}^{2}\left(\Omega_{2}\right)$ is the space of $L^{2}\left(\Omega_{2}\right)$ functions with zero mean. On the fluid-solid domain $\Omega=\Omega_{1} \cup \Omega_{2}$, we denote the trial and test function spaces for the temperature $T_{f}$ as

$$
\begin{aligned}
& H^{1}\left(\Omega_{2}\right)=\left\{T \in H^{1}\left(\Omega_{2}\right) \mid T=T_{0} \text { on } \Gamma_{i}\right\} \\
& H_{0}^{1}\left(\Omega_{2}\right)=\left\{T \in H_{0}^{1}\left(\Omega_{2}\right) \mid T=0 \text { on } \Gamma_{i}\right\} .
\end{aligned}
$$

The trial and test function spaces for the temperature $T_{s}$ are each $H^{1}\left(\Omega_{1}\right)$.

In order to show the regularity of the boundary control profile in later sections, we impose the Dirichlet boundary condition at the control boundary weakly through the Lagrange multiplier $[5,26,66]$, which represents the stress at the corresponding boundary in the dual system. As an alternative, the penalty approach has also been used successfully in fluid control problems. See $[2,79]$. We now turn to the derivation of the weak form for the fluid motion equations (2.1), and we look for $\mathbf{u} \in \mathbf{H}^{1}\left(\Omega_{2}\right), p \in L_{0}^{2}\left(\Omega_{2}\right)$ and $\sigma \in \mathbf{H}^{-1 / 2}\left(\Gamma_{i}\right)$ such that

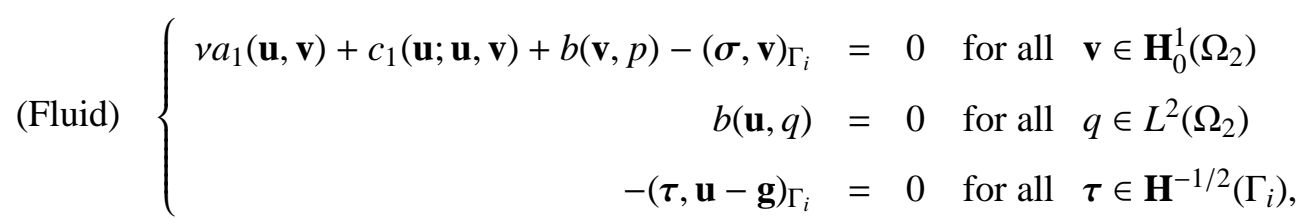


where

$$
\begin{aligned}
& a_{1}(\mathbf{u}, \mathbf{v}):=\frac{1}{2} \int_{\Omega_{2}}\left(\nabla \mathbf{u}+\nabla \mathbf{u}^{T}\right):\left(\nabla \mathbf{v}+\nabla \mathbf{v}^{T}\right) d \mathbf{x} \\
& c_{1}(\mathbf{u} ; \mathbf{u}, \mathbf{v}):=\int_{\Omega_{2}}(\mathbf{u} \cdot \nabla \mathbf{u}) \mathbf{v} d \mathbf{x} \\
& b(\mathbf{v}, p):=-\int_{\Omega_{2}}(\nabla \cdot \mathbf{v}) p d \mathbf{x} .
\end{aligned}
$$

We note that $\sigma$ is the stress at the control boundary $\Gamma_{i}$ in the state system .

In the weak forms of the fluid-solid internal energy equations (2.2)-(2.3), we look for $T_{f} \in$ $H^{1}\left(\Omega_{2}\right)$ and $T_{s} \in H^{1}\left(\Omega_{1}\right)$ such that

$$
\begin{array}{llll}
\text { (Fluid) } \quad k_{f} a_{2}\left(T_{f}, \phi\right)+c_{2}\left(\mathbf{u} ; T_{f}, \phi\right) & =0 & \text { for all } \phi \in H_{0}^{1}\left(\Omega_{2}\right) \\
\text { (Solid) } & k_{s} a_{3}\left(T_{s}, \psi\right)-(q, \psi)_{\Omega_{1}} & =0 & \text { for all } \psi \in H^{1}\left(\Omega_{1}\right),
\end{array}
$$

where

$$
\begin{aligned}
& a_{2}\left(T_{f}, \phi\right):=\int_{\Omega_{2}} \nabla T_{f} \cdot \nabla \phi d \mathbf{x} \\
& c_{2}\left(\mathbf{u} ; T_{f}, \phi\right):=\int_{\Omega_{2}}\left(\mathbf{u} \cdot \nabla T_{f}\right) \phi d \mathbf{x} \\
& a_{3}\left(T_{s}, \psi\right):=\int_{\Omega_{1}} \nabla T_{s} \cdot \nabla \psi d \mathbf{x} .
\end{aligned}
$$

\subsection{First Order Optimality Conditions}

We have assumed that $\mathbf{g}$ belongs to $\mathbf{H}^{1}\left(\Gamma_{i}\right)$ and has a bounded $\mathbf{H}^{1}$ norm in Section 2.3. Consequently, for the objective function (2.4), there is a minimizing sequence of $\left(\mathbf{u}, p, T_{f}, T_{s}, \mathbf{g}\right)^{T}$ in the product space $\mathbf{H}^{1}\left(\Omega_{2}\right) \times L_{0}^{2}\left(\Omega_{2}\right) \times H^{1}\left(\Omega_{2}\right) \times H^{1}\left(\Omega_{1}\right) \times \mathbf{H}^{1}\left(\Gamma_{i}\right)$, where $\left(\mathbf{u}, p, T_{f}, T_{s}\right)^{T}$ solves the state equations (2.1)-(2.3) and is bounded in $\mathbf{H}^{1}\left(\Omega_{2}\right) \times L_{0}^{2}\left(\Omega_{2}\right) \times H^{1}\left(\Omega_{2}\right) \times H^{1}\left(\Omega_{1}\right)$. Therefore, an optimal solution exists for the Dirichlet boundary control of the CHT process (2.1)-(2.4). To characterize the optimality conditions of the system (2.1)-(2.4), we introduce the Lagrange multipliers $\left(\boldsymbol{\xi}, \mu, \tau, \theta_{f}, \theta_{s}\right)^{T}$ for the state variables $\left(\mathbf{u}, p, \sigma, T_{f}, T_{s}\right)^{T}$. Taking $\Omega_{d}=\Gamma_{c}$, we define the Lagrangian function for the 


\section{CHAPTER 4. OPTIMALITY CONDITIONS IN INFINITE DIMENSIONAL SPACES}

objective function (2.4) and the state equations (4.3) and (4.5) as

$$
\begin{aligned}
\mathcal{L}\left(\mathbf{u}, p, \boldsymbol{\sigma}, T_{f}, T_{s},\right. & \left.\xi, \mu, \tau, \theta_{f}, \theta_{s}\right):=\frac{1}{2}\left(T_{f}-T_{d}, T_{f}-T_{d}\right)_{\Gamma_{c}}+\frac{\beta_{1}}{2}(\mathbf{g}, \mathbf{g})_{\Gamma_{i}}+\frac{\beta_{2}}{2}(\nabla \mathbf{g}, \nabla \mathbf{g})_{\Gamma_{i}} \\
& +v a_{1}(\mathbf{u}, \boldsymbol{\xi})+c_{1}(\mathbf{u} ; \mathbf{u}, \boldsymbol{\xi})+b(\boldsymbol{\xi}, p)-(\boldsymbol{\sigma}, \boldsymbol{\xi})_{\Gamma_{i}}+b(\mathbf{u}, \mu)-(\boldsymbol{\tau}, \mathbf{u}-\mathbf{g})_{\Gamma_{i}} \\
& +k_{f} a_{2}\left(T_{f}, \theta_{f}\right)+c_{2}\left(\mathbf{u} ; T_{f}, \theta_{f}\right) \\
& +k_{s} a_{3}\left(T_{s}, \theta_{s}\right)-\left(q, \theta_{s}\right)_{\Omega_{1}} .
\end{aligned}
$$

First, by taking variations of the Lagrangian $\mathcal{L}$ with respect to the multipliers $\left(\boldsymbol{\xi}, \mu, \tau, \theta_{f}, \theta_{s}\right)^{T}$ and requiring stationarity of the Lagrangian at local extrema, we recover the state equations (4.3) and (4.5). Similarly, by taking variations of the Lagrangian $\mathcal{L}$ with respect to the state variables $(\mathbf{u}, p, \sigma)^{T}$ along the direction $(\tilde{\mathbf{u}}, \tilde{p}, \tilde{\boldsymbol{\sigma}})^{T}$, we obtain the adjoint Navier-Stokes equations

$$
\text { (Fluid) }\left\{\begin{aligned}
v a_{1}(\tilde{\mathbf{u}}, \boldsymbol{\xi})+c_{1}(\tilde{\mathbf{u}} ; \mathbf{u}, \boldsymbol{\xi})+c_{1}(\mathbf{u} ; \tilde{\mathbf{u}}, \boldsymbol{\xi})+b(\tilde{\mathbf{u}}, \mu)-(\tau, \tilde{\mathbf{u}})_{\Gamma_{i}}+c_{2}\left(\tilde{\mathbf{u}} ; T_{f}, \theta_{f}\right) & =0 \\
b(\boldsymbol{\xi}, \tilde{p}) & =0 \\
-(\tilde{\boldsymbol{\sigma}}, \boldsymbol{\xi})_{\Gamma_{i}} & =0
\end{aligned}\right.
$$

for all $(\tilde{\mathbf{u}}, \tilde{p}, \tilde{\boldsymbol{\sigma}})^{T} \in \mathbf{H}_{0}^{1}\left(\Omega_{2}\right) \times L^{2}\left(\Omega_{2}\right) \times \mathbf{H}^{-1 / 2}\left(\Gamma_{i}\right)$.

Moreover, by taking variations of the Lagrangian $\mathcal{L}$ with respect to the state variables $\left(T_{f}, T_{s}\right)^{T}$ along the direction $\left(\tilde{T}_{f}, \tilde{T}_{s}\right)^{T}$, we obtain the adjoint internal energy equations

$$
\begin{array}{llll}
\text { (Fluid) } & k_{f} a_{2}\left(\tilde{T}_{f}, \theta_{f}\right)+c_{2}\left(\mathbf{u} ; \tilde{T}_{f}, \theta_{f}\right)+\left(T_{f}-T_{d}, \tilde{T}_{f}\right)_{\Gamma_{c}} & =0 & \text { for all } \tilde{T}_{f} \in H_{0}^{1}\left(\Omega_{2}\right) \\
\text { (Solid) } & k_{s} a_{3}\left(\tilde{T}_{s}, \theta_{s}\right) & =0 & \text { for all } \tilde{T}_{s} \in H^{1}\left(\Omega_{1}\right) .
\end{array}
$$

Finally, we take variations of the Lagrangian $\mathcal{L}$ with respect to the variable $\mathbf{g}$ along the direction $\tilde{\mathbf{g}}$ to obtain the reduced gradient equation

$$
(\text { Gradient }) \quad \beta_{1}(\mathbf{g}, \tilde{\mathbf{g}})_{\Gamma_{i}}+\beta_{2}(\nabla \mathbf{g}, \nabla \tilde{\mathbf{g}})_{\Gamma_{i}}+(\boldsymbol{\tau}, \tilde{\mathbf{g}})_{\Gamma_{i}}=0 \quad \text { for all } \quad \tilde{\mathbf{g}} \in \mathbf{H}^{1 / 2}\left(\Gamma_{i}\right)
$$

Here, as indicated in the system (4.3), $\tau$ belongs to the space $\mathbf{H}^{-1 / 2}\left(\Gamma_{i}\right)$. For more details on the derivation of the Optimality Conditions, see Appendix A. The optimality system of the Dirichlet velocity boundary control of the CHT process is fully coupled and consists of the nonlinear state equations (4.3) and (4.5), the adjoint equations (4.8)-(4.9), and the reduced gradient equation (4.10). 


\section{CHAPTER 4. OPTIMALITY CONDITIONS IN INFINITE DIMENSIONAL SPACES}

In order to see the structural similarity between the state and the adjoint systems, we need to derive the strong form of the adjoint equations. We write the adjoint Navier-Stokes equations in strong form

$$
\text { (Fluid) }\left\{\begin{array}{rllll}
-\nu \nabla \cdot\left(\nabla \boldsymbol{\xi}+\nabla \boldsymbol{\xi}^{T}\right)+(\nabla \mathbf{u})^{T} \boldsymbol{\xi}-(\mathbf{u} \cdot \nabla) \boldsymbol{\xi}+\nabla \mu+\nabla T_{f} \theta_{f} & =0 & \text { in } & \Omega_{2} \\
\nabla \cdot \boldsymbol{\xi} & = & 0 & \text { in } & \Omega_{2} \\
\boldsymbol{\xi} & = & 0 & \text { on } & \Gamma_{i}, \Gamma_{c}, \Gamma_{4} \\
-\mu \mathbf{n}+\nu\left(\nabla \boldsymbol{\xi}+\nabla \boldsymbol{\xi}^{T}\right) \mathbf{n}+(\mathbf{u} \cdot \mathbf{n}) \boldsymbol{\xi} & = & 0 & \text { on } & \Gamma_{o} \\
\mathbf{n} \cdot\left(-\mu \mathbf{I}+v\left(\nabla \boldsymbol{\xi}+\nabla \boldsymbol{\xi}^{T}\right)\right)-\boldsymbol{\tau} & = & 0 & \text { on } & \Gamma_{i},
\end{array}\right.
$$

where $\boldsymbol{\xi}=\boldsymbol{\xi}(x, y)$ is the adjoint velocity, $\mu$ is the adjoint pressure, and $\boldsymbol{\tau}$ is the adjoint stress at the inflow boundary $\Gamma_{i}$. The appropriate Sobolev spaces are $(\xi, \mu, \tau)^{T} \in \mathbf{H}_{0}^{1}\left(\Omega_{2}\right) \times L^{2}\left(\Omega_{2}\right) \times \mathbf{H}^{-1 / 2}\left(\Gamma_{i}\right)$ [66]. We write the adjoint fluid-solid internal energy equations in strong form

$$
\text { (Fluid) }\left\{\begin{array}{rllll}
-k_{f} \Delta \theta_{f}-\mathbf{u} \cdot \nabla \theta_{f} & =0 & \text { in } & \Omega_{2} \\
\mathbf{n} \cdot\left(k_{f} \nabla \theta_{f}-k_{s} \nabla \theta_{s}\right)-\left(T_{f}-T_{d}\right) & =0 & \text { on } & \Gamma_{c} \\
\theta_{f} & =\theta_{s} & \text { on } & \Gamma_{c} \\
\mathbf{n} \cdot \nabla \theta_{f} & =0 & \text { on } & \Gamma_{4} \\
\mathbf{n} \cdot k_{f} \nabla \theta_{f}+u \theta_{f} & =0 & \text { on } & \Gamma_{o} \\
\theta_{f} & =0 & \text { on } & \Gamma_{i},
\end{array}\right.
$$

and

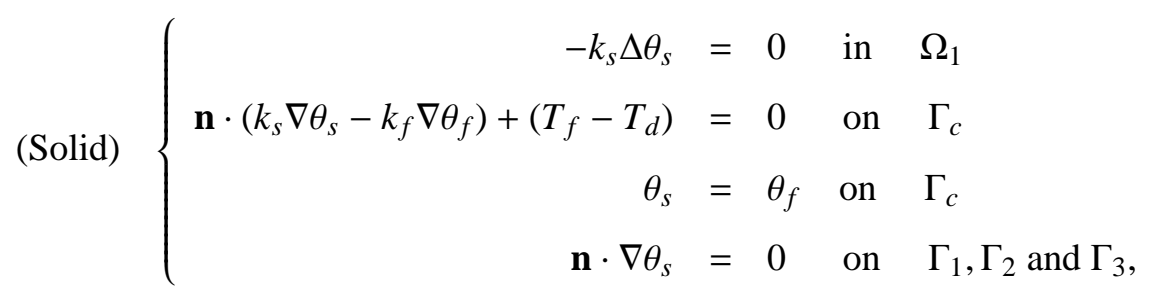

where $\left(\theta_{f}, \theta_{s}\right)^{T} \in H_{0}^{1}\left(\Omega_{2}\right) \times H^{1}\left(\Omega_{1}\right)$. In the derivation of (4.11) and (4.12), we have used the integral by parts formula on the term $c_{1}(\mathbf{u} ; \tilde{\mathbf{u}}, \boldsymbol{\xi})$ in (4.8) and the term $c_{2}\left(\mathbf{u} ; \tilde{T}_{f}, \theta_{f}\right)$ in (4.9). Comparing the strong forms of the adjoint equations (4.11)-(4.13) with the strong forms of the state equations (2.1)-(2.3), it is evident that these two sets of equations have similar structures, but with convective "winds" blowing from opposite directions. More specifically, in the adjoint systems, the "wind" 
is $-\mathbf{u}$ and the boundary $\Gamma_{o}$ becomes the inlet. Furthermore, the structural similarity between the state and the adjoint systems indicates that, in the numerical implementation, it is very convenient to modify the code for solving the state equations into that for adjoint equations.

Finally, we write the gradient equation (4.10) in the strong form

$$
\text { (Gradient) } \quad-\beta_{2} \Delta \mathbf{g}+\beta_{1} \mathbf{g}+\boldsymbol{\tau}=0 \quad \text { on } \quad \Gamma_{i}
$$

We note that in the Steepest Descent method, the algorithm starts from solving the state equations (2.1), (2.2), (2.3) for (u, $\left.p, \sigma, T_{f}, T_{s}\right)^{T}$, then solves the adjoint equations (4.11), (4.12), (4.13) for $\left(\xi, \mu, \tau, \theta_{f}, \theta_{s}\right)^{T}$, and finally updates the control profile along the negative gradient direction in (4.14).

\subsection{Necessity of $\beta_{2}>0$ in the Continuous Problem}

In [65] and [43], it has been pointed out that a nonzero $\beta_{2}$ is suitable for deriving regularity results for the optimal control of Navier-Stokes equations and for generating a less oscillary control. Indeed, because $\tau$ is the boundary stress of the adjoint Navier-Stokes equations and belongs to $\in \mathbf{H}^{-1 / 2}\left(\Gamma_{i}\right)$ in (4.11), and $\beta_{2}>0$ in the gradient condition (4.14), if we enforce the homogenous boundary condition for $\mathbf{g}$ on the boundary $\Gamma_{i}$ in (4.14), it can be shown via the Lax-Milgram theorem that $\mathbf{g} \in \mathbf{H}^{3 / 2}\left(\Gamma_{i}\right)$ [58]. This indicates that $\mathbf{g}$ even lies in a subspace of $\mathbf{H}^{1}\left(\Gamma_{i}\right)$.

If we had imposed $\beta_{2}=0$ in (2.4), the control problem (2.1)-(2.4) would be ill posed, because

merely having the term $\frac{\beta_{1}}{2} \int_{\Gamma_{i}}|\mathbf{g}|^{2} d s$ in the objective function (2.4) is insufficient for providing a bounded control $\mathbf{g}$ in $\mathbf{H}^{1 / 2}\left(\Gamma_{i}\right)$, which, however, is a part of the minimal regularity requirement known for the existence of solutions to Navier-Stokes equations. Therefore, in infinite dimensional function spaces, the regularization term on the control gradient is necessary for the existence of solutions to the Dirichlet boundary control of the CHT process. 


\section{Chapter 5}

\section{Discretization of the State and the Objective}

\subsection{Overview}

Based on the weak formulations (4.3) and (4.5), we carry out the Finite Element discretization for the state equations and the objective function. In Section 5.2, we discuss the proper discretization of the state equations and its Finite Element assembly in block matrix form. In Section 5.3, we discuss the discretization of the objective function and the necessity of $\beta_{2}>0$ in the discretized problem.

\subsection{Discrete State Equations}

In this multiphysics system, there are three phenomena, namely, the momentum transport in the fluid region, the convection-diffusion process of the fluid internal energy and the heat diffusion in the solid region. For the incompressible Navier-Stokes equations, we use the Galerkin formulation with the stable Taylor-Hood (Q2-Q1) element. Alternatively, stabilized elements can also be used [48]. For the fluid-solid internal energy equations, we use the Q2 Lagrange finite element with piecewisequadratic basis functions. In the mesh generation, the fluid-solid domain $\Omega=\Omega_{1} \cup \Omega_{2}$ is first partitioned into uniform quadrilateral cells for Q2 elements, and then a layer of Q1 macro-element 
cells is attached to the fluid domain. The resulting Q2-Q1 discretization for $(\mathbf{u}, p)^{T}$ and the Q2 discretization for $\left(T_{f}, T_{s}\right)^{T}$ leads to an approximation that allows comparable accuracy between the velocity and temperature variables [46].

The weak forms (4.3) and (4.5) of the state equations are nonlinear, due to the couplings in the velocity components $\left(u_{x}, u_{y}\right)$ and in the velocity-temperature pair $\left(\mathbf{u}, T_{f}\right)$. We apply Newton's method to this nonlinear system. At the iterate $\left(\mathbf{u}^{k}, p^{k}, \sigma^{k}, T_{f}^{k}, T_{s}^{k}\right)^{T}$, we write the Newton system of (4.3) and (4.5) for $\left(\delta \mathbf{u}, \delta p, \delta \sigma, \delta T_{f}, \delta T_{s}\right)^{T}$ as

$$
\text { (Fluid) }\left\{\begin{aligned}
v a_{1}(\delta \mathbf{u}, \mathbf{v})+c_{1}(\delta \mathbf{u} ; \mathbf{u}, \mathbf{v})+c_{1}(\mathbf{u} ; \delta \mathbf{u}, \mathbf{v})+b(\mathbf{v}, \delta p)-(\delta \sigma, \mathbf{v})_{\Gamma_{i}} & =R_{1} \\
b(\delta \mathbf{u}, q) & =R_{2} \\
-(\tau, \delta \mathbf{u})_{\Gamma_{i}} & =R_{3},
\end{aligned}\right.
$$

for all $(\mathbf{v}, q, \tau)^{T} \in \mathbf{H}_{0}^{1}\left(\Omega_{2}\right) \times L^{2}\left(\Omega_{2}\right) \times \mathbf{H}^{-1 / 2}\left(\Gamma_{i}\right)$, and

$$
\begin{aligned}
& \text { (Fluid) } \quad k_{f} a_{2}\left(\delta T_{f}, \phi\right)+c_{2}\left(\delta \mathbf{u} ; T_{f}, \phi\right)+c_{2}\left(\mathbf{u} ; \delta T_{f}, \phi\right)=R_{4} \quad \text { for all } \phi \in H_{0}^{1}\left(\Omega_{2}\right) \\
& \text { (Solid) } k_{s} a_{3}\left(\delta T_{s}, \psi\right) \quad=R_{5} \text { for all } \psi \in H^{1}\left(\Omega_{1}\right) \text {, }
\end{aligned}
$$

where the superscript $k$ is omitted to simplify the notation. At iteration $k+1$, we update the state variable

$$
\left(\mathbf{u}^{k+1}, p^{k+1}, \boldsymbol{\sigma}^{k+1}, T_{f}^{k+1}, T_{s}^{k+1}\right)^{T}=\left(\mathbf{u}^{k}, p^{k}, \boldsymbol{\sigma}^{k}, T_{f}^{k}, T_{s}^{k}\right)^{T}+\left(\delta \mathbf{u}, \delta p, \delta \boldsymbol{\sigma}, \delta T_{f}, \delta T_{s}\right)^{T}
$$

We introduce the mesh size $h$-dependent finite element basis functions for the velocity, pressure, boundary stress, fluid temperature and solid temperature:

$$
\begin{aligned}
& \text { for } \mathbf{u}: \mathbf{V}_{h}=\left\{\left[\begin{array}{l}
\phi_{i} \\
0
\end{array}\right],\left[\begin{array}{l}
0 \\
\phi_{i}
\end{array}\right]\right\}_{i=1, \ldots, n_{u}} \in \mathbf{H}^{1}\left(\Omega_{2}\right) ; \\
& \text { for } \boldsymbol{\sigma}: \mathbf{S}_{h}=\left\{\left[\begin{array}{l}
s_{i} \\
0
\end{array}\right],\left[\begin{array}{l}
0 \\
s_{i}
\end{array}\right]\right\}_{i=1, \ldots, n_{\Gamma_{i}}} \in \mathbf{H}^{1}\left(\Omega_{2}\right) ; \\
& \text { for } p: \mathbf{P}_{h}=\left\{\psi_{i}\right\}_{i=1, \ldots, n_{p}} \in L^{2}\left(\Omega_{2}\right) ; \\
& \text { for } T_{f}: \mathbf{T}_{f_{h}}=\left\{\varphi_{f_{i}}\right\}_{i=1, \ldots, n_{T_{f}}} \in H^{1}\left(\Omega_{2}\right) ; \\
& \text { for } T_{s}: \mathbf{T}_{s h}=\left\{\varphi_{s i}\right\}_{i=1, \ldots, n_{T_{s}}} \in H^{1}\left(\Omega_{1}\right),
\end{aligned}
$$


where $n_{u}, n_{p}, n_{\Gamma_{i}}, n_{T_{f}}$, and $n_{T_{s}}$ denote the degrees of freedom in the velocity, pressure, boundary stress, fluid temperature and solid temperature approximations. With the finite element approximation of $\left(\mathbf{u}, p, \sigma, T_{f}, T_{s}\right)$ known at iteration $k$, we expand the Newton update $\left(\delta \mathbf{u}, \delta p, \delta \sigma, \delta T_{f}, \delta T_{s}\right)$ in the finite element basis functions above:

$$
\begin{aligned}
& \delta \mathbf{u}=\left[\begin{array}{l}
\sum_{j=1}^{n_{u}} \phi_{j} \delta u_{x j} \\
\sum_{j=1}^{n_{u}} \phi_{j} \delta u_{y_{j}}
\end{array}\right] ; \quad \delta \boldsymbol{\sigma}=\left[\begin{array}{l}
\sum_{j=1}^{n_{\Gamma_{i}}} \varsigma_{j} \delta \sigma_{x j} \\
\sum_{j=1}^{n_{\Gamma_{i}}} \varsigma_{j} \delta \sigma_{y_{j}}
\end{array}\right] ; \\
& \delta p=\sum_{j=1}^{n_{p}} \psi_{j} \delta p_{j} ; \quad \delta T_{f}=\sum_{j=1}^{n_{T_{f}}} \varphi_{f_{j}} \delta T_{f_{j}} ; \\
& \delta T_{s}=\sum_{j=1}^{n_{T_{s}}} \varphi_{s j} \delta T_{s j} .
\end{aligned}
$$

After substituting the finite element approximation (5.4) into the Newton system (5.1) and (5.2), we obtain the Finite Element assembly of the state system:

$$
\left(\begin{array}{ccccc|cc}
A+N+W_{x x} & W_{x y} & B_{x}^{T} & \Pi_{x}^{T} & 0 & 0 & 0 \\
W_{y x} & A+N+W_{y y} & B_{y}^{T} & 0 & \Pi_{y}^{T} & 0 & 0 \\
B_{x} & B_{y} & 0 & 0 & 0 & 0 & 0 \\
\Pi_{x} & 0 & 0 & 0 & 0 & 0 & 0 \\
0 & \Pi_{y} & 0 & 0 & 0 & 0 & 0 \\
\hline M_{x} & M_{y} & 0 & 0 & 0 & A_{f}+N_{f} & 0 \\
0 & 0 & 0 & 0 & 0 & 0 & A_{s}
\end{array}\right)\left(\begin{array}{c}
\delta u_{x} \\
\delta u_{y} \\
\delta p \\
\delta \sigma_{x} \\
\delta \sigma_{y} \\
\delta T_{f} \\
\delta T_{s}
\end{array}\right),
$$


where the block matrices are defined as

$$
\begin{aligned}
& A=\left[a_{i j}\right], a_{i j}=v \int_{\Omega_{2}} \nabla \phi_{j} \cdot \nabla \phi_{i} ; \\
& B_{\alpha}=\left[b_{\alpha i j}\right], b_{\alpha i j}=-\int_{\Omega_{2}} \frac{\partial \phi_{j}}{\partial \alpha} \psi_{i} ; \\
& N=\left[n_{i j}\right], n_{i j}=\int_{\Omega_{2}}\left(u_{h x} \frac{\partial \phi_{j}}{\partial x}+u_{h y} \frac{\partial \phi_{j}}{\partial y}\right) \phi_{i} ; \\
& W_{\alpha \beta}=\left[w_{\alpha \beta i j}\right], w_{\alpha \beta i j}=\int_{\Omega_{2}} \frac{\partial u_{h \alpha}}{\partial \beta} \phi_{j} \phi_{i} ; \\
& \Pi_{\alpha}=\left[\pi_{\alpha i j}\right], \pi_{\alpha i j}=\int_{\Gamma_{i}} \phi_{j} \varsigma_{i} \\
& M_{\alpha}=\left[m_{\alpha i j}\right], m_{\alpha i j}=\int_{\Omega_{2}} \frac{\partial T_{f h}}{\partial \alpha} \phi_{j} \varphi_{f_{i}} \\
& A_{f}=\left[a_{f i j}\right], a_{f i j}=k_{f} \int_{\Omega_{2}}^{\nabla \varphi_{f}} \cdot \nabla \varphi_{f_{i}} .
\end{aligned}
$$

Here, $\alpha, \beta \in\{x, y\}$, and $\mathbf{u}_{h}=\left(u_{h x}, u_{h y}\right)^{T}$ denotes the mesh size $h$-dependent finite element approximations of the velocity $\mathbf{u}$. We note that in (5.5) there are tiny nonzero blocks in the $\left(\delta T_{f}, \delta T_{s}\right)$ and $\left(\delta T_{s}, \delta T_{f}\right)$ positions due to the connectivity of finite element cells at the fluid-solid interfacial boundary $\Gamma_{c}$. In other words, the block matrices $A_{f}+N_{f}$ and $A_{s}$ are not strictly decoupled.

In the setting of optimal control problems, a priori error estimates for Finite Element approximations of the state and the adjoint variables $(\mathbf{u}, p, \boldsymbol{\xi}, \mu)^{T}$ for Navier-Stokes equations are discussed in [64], and the estimate of $\left(T_{f}, T_{s}, \theta_{f}, \theta_{s}\right)^{T}$ for convection-diffusion equations are discussed in [63]. Finally, we conclude this section with the following remarks:

Remark 5.2.1. The first-order necessary optimality-conditions (4.3), (4.5), and (4.8)-(4.10) are the so-called Karush-Kuhn-Tucker (KKT) conditions in constrained optimization. When the KKT conditions are linearized with Newton's method and then discretized, they become a discrete linear KKT system with the block partitioning $(\{\mathbf{U}\},\{\mathbf{g}\},\{\lambda\})^{T}$. Here, the state variables $\mathbf{U}:=\left(\mathbf{u}, p, \sigma, T_{s}, T_{f}\right)^{T}$ and the adjoint variables $\lambda:=\left(\xi, \mu, \tau, \theta_{f}, \theta_{s}\right)^{T}$. This system is ill-conditioned and indefinite with positive and negative eigenvalues. The solution methods of the KKT conditions are classified into two categories, namely the full-space and the reduced-space approaches. In the full-space approach, Newton's method combined with Krylov solvers can be exploited to solve simultaneously for $(\{\mathbf{U}\},\{\mathbf{g}\},\{\lambda\})^{T}$ from the coupled KKT system. An effective preconditioner for the KKT system is 


\section{CHAPTER 5. DISCRETIZATION OF THE STATE AND THE OBJECTIVE}

the key component to obtain an efficient and effective optimization scheme in the full space. On the other hand, in the reduced-space approach, either the block Gaussian elimination or solution-space decomposition technique can be utilized to decouple the KKT system into separated linear systems for each of the state, adjoint, and control variables. The coefficient matrix of the resulting decoupled gradient equation is the reduced Hessian, which is dense, but much smaller than the KKT matrix, and has the same dimension as the control space. See [3].

In general, solving the KKT system is equivalent to applying the Sequential Quadratic Programming (SQP) technique to the original optimization problem (2.1)-(2.4). Therefore, the full-space and the reduced-space approaches are also referred to as the FSQP and the RSQP methods respectively. The full-space approach is attractive for several reasons. First, the state, adjoint, and control variables are solved simultaneously and inexactly towards optimal solutions. In fact, the state and adjoint constraints are satisfied exactly only at the final optimization iteration. Second, the inexactness of the solution process makes full-space approaches very efficient, because at the early stage of the optimization loop the control variables are far from optimal, and approximate solutions of the state and adjoint variables are normally sufficient for obtaining a descent search direction in the full space. Finally, the preconditioners of the coupled KKT matrix can be generated by reusing existing state and adjoint solvers. The main difficulty of the full-space approach lies in the complexity of obtaining an efficient preconditioner for the coupled, indefinite, and illconditioned KKT system. Recently, there have been several successful applications based on the full-space approach. See [3,26,111,122] and references therein. The scalability comparisons of the full-space and reduced-space approaches are also discussed in [3]. The exploration of inexact Newton's methods for solving the KKT system is discussed for full-space approach in [54] and reduced-space approach in $[24,71,82]$.

Remark 5.2.2. In this multiphysics CHT system, the three components, namely, the momentum transport inside the fluid, the convection-diffusion process of the fluid internal energy and the heat diffusion in the solid, are strongly coupled in the optimization algorithm. In fact, each time we update the boundary control $\mathbf{g}$, we need to solve the consistently coupled state equations for the state variables (u, $\left.p, T_{s}, T_{f}\right)$. Nevertheless, computationally, this solution process can be decoupled. 
CHAPTER 5. DISCRETIZATION OF THE STATE AND THE OBJECTIVE

First, we can solve for the velocity-pressure pair $(\mathbf{u}, p)$ from the Navier-Stokes equations. This in turn allows us to solve a linear system for the temperature variables $\left(T_{s}, T_{f}\right)$. The advantages of this numerical decoupling are threefold. It allows us to reduce the nonlinearity of the coupled system by solving each physics separately. In addition, the memory usage of the segregated approach is much less than that of the coupled approach for both the state and the adjoint equations. Finally, the computational separation of different physics provides the possibility of reusing existing state equation solvers. This segregated approach is a variant of the Reduced SQP method as shown in Appendix $C$. The price for the segregated approach would be several more nonlinear optimization iterations in the reduced control space than Newton applied to the coupled KKT system. In this study, we have implemented the segregated solvers.

\subsection{Necessity of the Smoothness Term in the Discrete Objective Func- tion}

On the continuous level, when $\beta_{2}=0$, the boundedness of $\mathbf{g}$ in $\mathbf{H}^{1 / 2}\left(\Gamma_{i}\right)$ is not guaranteed, and the solution to Navier-Stokes equations (2.1) may not exist. See Section 4.4. On the other hand, on the discrete level, because all norms in finite dimensional spaces are equivalent, $\beta_{2}=0$ can be freely chosen for a fixed discretization to ensure the well-posedness of the discretized optimal control problem. However, in the test cases of this study, we emphasize that we have to choose $\beta_{2}>0$ in the discrete objective function to keep it consistent with the continuous formulation (2.1)-(2.4). Otherwise, only nonsmooth and nonphysical controls can be generated by the numerical algorithms. The evidence is shown in Section 7.2.1. Therefore, the discrete objective function has the same form as (2.4) with $\beta_{1}, \beta_{2}>0$.

One way to see the necessity of $\beta_{2}>0$ in the discrete objective function is that if we have imposed $\beta_{2}=0$ on the discrete level, we have $\mathbf{g}=-\left(1 / \beta_{1}\right) \tau$ and $\mathbf{g} \in \mathbf{H}^{-1 / 2}\left(\Gamma_{i}\right)$ from (4.14). Moreover, since the finite element approximation for $\tau$ is not smooth in comparison with that for the velocity $\mathbf{u}$, which has the regularity estimate in $\mathbf{H}^{1}\left(\Omega_{2}\right)$, the finite element approximation for the control profile $\mathbf{g}$ is also not smooth. See Figure 7.2 in Section 7.2.1 for the nonsmoothness of $\mathbf{g}$ 
when $\beta_{2}=0$.

In fact, it is not enough to merely have $\beta_{2}>0$ in the continuous and the discretized control problems for the optimization algorithm to locate the correct optimal solutions. Numerical smoothing [83-85] has to be involved in the gradient computation to enhance the regularity of computed control profiles. See Section 6.3. Furthermore, as shown in the numerical test problem in Section 7.2.1 and 7.2.2, the condition $\beta_{2}>0$ in the objective function has also been computationally necessary for deriving the numerical smoothing operator and increasing the regularity of the control profile $\mathbf{g}$. 


\section{Chapter 6}

\section{Numerical Optimization Algorithms}

\subsection{Overview}

The complex structure of the optimality conditions (4.3), (4.5), (4.8), (4.9) and (4.10) for the Dirichlet velocity boundary control of the CHT process has made analytical solutions impossible for nontrivial target temperature profiles; therefore, we seek numerical approximations to the exact optimal control solution. Moreover, as shown in Section 5.3, imposing regularization on the $\nabla \mathbf{g}$ term in the continuous and the discrete objective functions (2.4) only guarantees a smooth control profile in the continuous theory, but not in the numerical setting. In fact, numerical smoothing devices that are directly applied to the control update must be incorporated into the optimization scheme. It is evident from the Numerical Test Case 1 in the Section 7.2.2 that when $\beta_{2}>0$, the optimization scheme using the plain Steepest Descent update on the control profile stagnates, due to the nonsmoothness of the control updates, which forces the line search iteration to take tiny steps at every nonlinear optimization iteration.

We discuss various approaches to obtaining the discrete optimality conditions in Section 6.2. In Section 6.3, based on the plain Steepest Descent method, we introduce the numerical smoothing device. In Section 6.4, for the L-BFGS method, we illustrate the implicit smoothing process, which is turned on at each Quasi-Newton Hessian update and makes this method successful. Finally, in Section 6.5, we combine the smoothed optimization scheme with the numerical continuation 
technique on regularization parameters to obtain a robust solution strategy for difficult boundary optimal control problems.

\section{2 "Discretize Then Optimize"}

There are two approaches for solving the optimality system, namely, the "optimize then discretize" approach and the "discretize then optimize" approach $[2,3,36]$. In the "optimize then discretize" approach, we discretize the state equations (4.3) and (4.5) and the adjoint equations (4.8)-(4.9), separately, and the discretization on the state and the adjoint equations can be done differently, namely, using either different methods, such as the Finite Element method or the Finite Volume method, or the same method but with different stabilization terms or different trial function spaces $[2,36]$. In contrast, in the "discretize then optimize" approach, we discretize the PDE state equations, introduce discrete multiplier functions acting on the discrete state equations, and then take variations of the discrete Lagrangian with respect to the discrete state variables in the finite dimensional space. At the end of this process, we obtain the discrete adjoint system and the discrete gradient condition. We note that these two approaches are generally not equivalent, since differentiation and discretization operators generally do not commute, especially for nonlinear problems or linear problems that have strong convection and nonlinear stabilization terms inside the discretization operator. For diffusion-convection-reaction equations discretized by discontinuous Galerkin methods, both approaches produce the same discrete optimality conditions for the symmetric interior penalty Galerkin (SIPG) method, but different optimality conditions for the nonsymmetric interior penalty Galerkin (NIPG) method or the incomplete interior penalty Galerkin (IIPG) method [134]. For convection-diffusion-reaction equations discretized with some stabilized finite element schemes such as the Local Projection Stabilization (LPS) method [16,30], the "optimize then discretize" and "discretize then optimize" approaches coincide and have optimal order of convergence for the state, adjoint, and control variables [17]. For the convection-diffusion equation with high order Galerkin discretization, it has been shown that the "optimize then discretize" approach produces better control solutions [36]. For the Oseen equations discretized by Finite Element methods with Galerkin/LeastSquares (GLS) stabilization, both approaches produce comparable approximations for the Dirichlet 
boundary control problem [2]. For other systems, a conclusive comparison between those two approaches has not been made, and we believe that the result is problem dependent. We also note that the discrete system in the "discretize then optimize" approach is generally symmetric for the state and the adjoint blocks, but this symmetry is lost for the "optimize then discretize" approach. In this study, we have carried out the "discretize then optimize" approach.

\subsection{Smoothed Steepest Descent Method}

For optimization with partial differential equation (PDE) systems as constraints, it is not rare that the second order information of the objective function is too complex or too expensive to obtain. However, the first order adjoint-based $[28,62,76]$ gradient information is usually accessible, given that the adjoint equations have a structure similar to that of the state equations and can be solved without too much modification of the state solvers. With an initial control vector $\mathbf{g}$ and the discretization in Chapter 5, we can solve for $\left(\mathbf{u}, p, T_{s}, T_{f}\right)^{T}$ from the state equations (4.3) and (4.5). With the state variables $\mathbf{u}$ and $T_{f}$ known, we can then solve the adjoint equations (4.8) and (4.9) for $\left(\boldsymbol{\xi}, \mu, \theta_{s}, \theta_{f}, \boldsymbol{\tau}\right)^{T}$. Finally, we obtain the Steepest Descent direction, which is the negative reduced gradient of the objective function $\mathcal{J}(\mathbf{g})$, namely, $-\left(\boldsymbol{\tau}+\beta_{1} \mathbf{g}-\beta_{2} \Delta \mathbf{g}\right)$ in (4.14). Here, $\mathcal{J}(\mathbf{g}):=\mathcal{J}\left(\mathbf{u}(\mathbf{g}), p(\mathbf{g}), T_{f}(\mathbf{g}), T_{s}(\mathbf{g}), \mathbf{g}\right)$ is the implicitly constrained objective function (2.4), and the state variables $\left(\mathbf{u}(\mathbf{g}), p(\mathbf{g}), T_{f}(\mathbf{g}), T_{s}(\mathbf{g})\right)^{T}$ are actuated by the control $\mathbf{g}$. See [72] for the framework of implicit constrained formulation. Moreover, this implicit constrained formulation can be viewed as a variant of the Reduced SQP method. See Appendix C.

To ensure that $\mathcal{J}(\mathbf{g})$ has sufficient decrease along the search direction, globalization techniques, such as line search or trust region methods [104], are required to generate feasible stepsizes. In this study, we have applied the line search method with only the Armijo condition (or the sufficient decrease condition). We note that for difficult problems, in addition to the sufficient decrease condition, the line search process can be further guarded by the curvature condition. However, the curvature condition requires the calculation of the gradient of the objective function at each line search iteration, which involves a pair of solves for the state and the adjoint equations, and thus is computationally too expensive. We summarize the Steepest Descent method in Algorithm 6.3.1. 


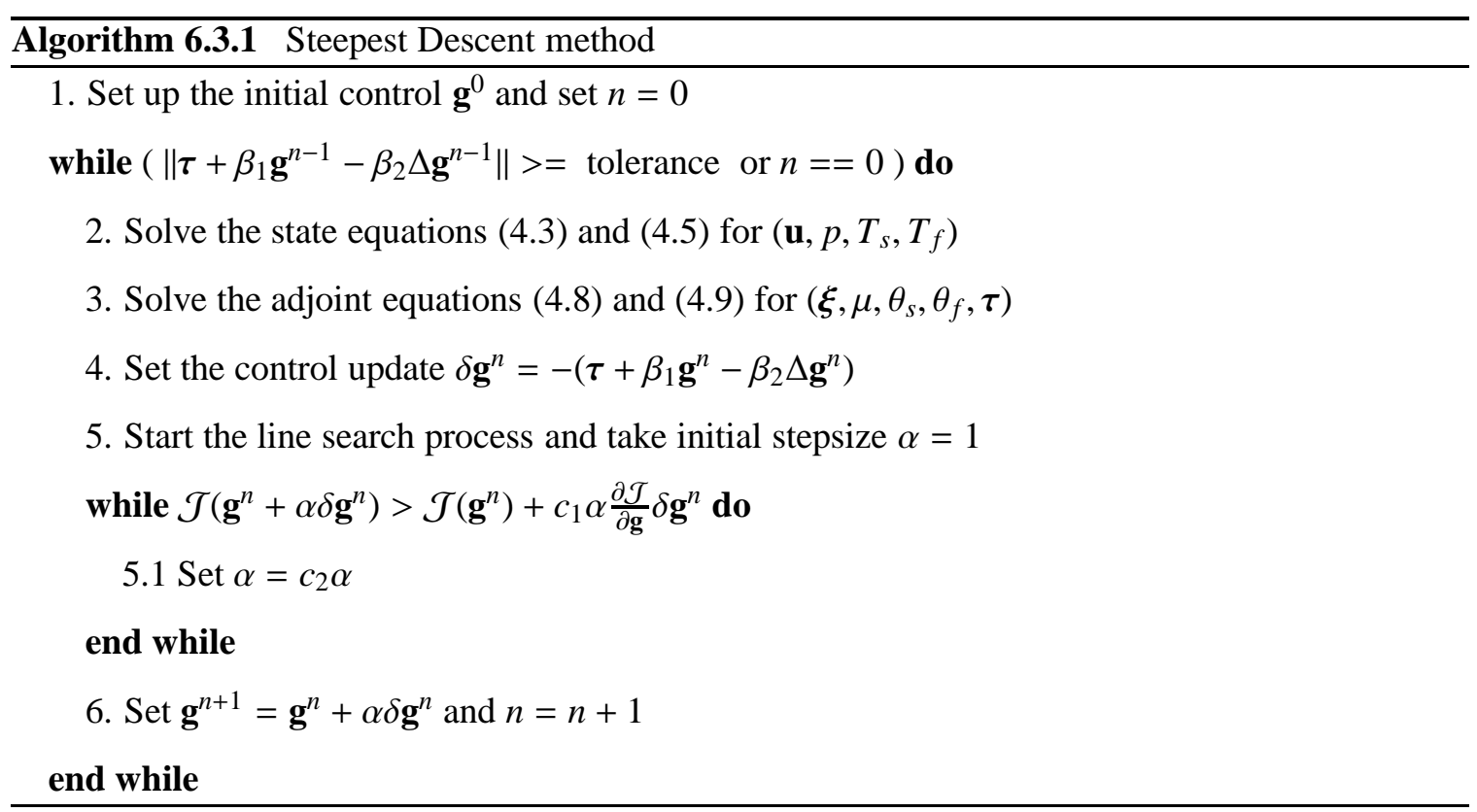

At the beginning of Section 6.1, we have pointed out the nonsmoothness of the numerical approximation of the control update, which restricts the line search procedure back up to tiny steps and causes the nonlinear optimization algorithm to stagnate. To circumvent this difficulty, we have implemented a simple smoothing device acting on the control update $\delta \mathbf{g}$. Starting from control $\mathbf{g}^{n}$, we solve the state and the adjoint equations. Instead of taking the Steepest Descent direction in Algorithm 6.3.1, we then set $\mathbf{g}^{n+1}=\mathbf{g}^{n}+\delta \mathbf{g}^{n}$ and enforce the decision equation (4.14) at iterate $\mathbf{g}^{n+1}$ :

$$
\begin{array}{ll}
\boldsymbol{\tau}+\beta_{1} \mathbf{g}^{n+1}-\beta_{2} \Delta \mathbf{g}^{n+1} & =0 \\
\boldsymbol{\tau}+\beta_{1}\left(\mathbf{g}^{n}+\delta \mathbf{g}^{n}\right)-\beta_{2} \Delta\left(\mathbf{g}^{n}+\delta \mathbf{g}^{n}\right) & =0 \\
\delta \mathbf{g}^{n}=-\left(\beta_{1} \mathbf{I}-\beta_{2} \Delta\right)^{-1}\left(\boldsymbol{\tau}+\beta_{1} \mathbf{g}^{n}-\beta_{2} \Delta \mathbf{g}^{n}\right),
\end{array}
$$

where (6.2) can be solved as a homogenous boundary value problem (BVP) on the Dirichlet control boundary $\Gamma_{i}$. In fact, the resolvent operator $\left(\beta_{1} \mathbf{I}-\beta_{2} \Delta\right)^{-1}$ in (6.2) has the smoothing effect on the Steepest Descent direction. One way to see this is to look into the auxiliary linear system $\mathbf{A x}=\mathbf{b}$, where $\mathbf{A}$ is a finite difference approximation of the operator $\beta_{1} \mathbf{I}-\beta_{2} \Delta$ in one dimension using 5 
mesh points. To compute $\mathbf{x}=\mathbf{A}^{-1} \mathbf{b}$, we use the Jacobi iterative method on the equation $\mathbf{A x}=\mathbf{b}$, namely the linear system

$$
\left(\begin{array}{cccc}
\beta_{1}+2 \frac{\beta_{2}}{h^{2}} & -\frac{\beta_{2}}{h^{2}} & 0 & 0 \\
-\frac{\beta_{2}}{h^{2}} & \beta_{1}+2 \frac{\beta_{2}}{h^{2}} & -\frac{\beta_{2}}{h^{2}} & 0 \\
0 & -\frac{\beta_{2}}{h^{2}} & \beta_{1}+2 \frac{\beta_{2}}{h^{2}} & -\frac{\beta_{2}}{h^{2}} \\
0 & 0 & -\frac{\beta_{2}}{h^{2}} & \beta_{1}+2 \frac{\beta_{2}}{h^{2}}
\end{array}\right)\left(\begin{array}{l}
x_{1} \\
x_{2} \\
x_{3} \\
x_{4}
\end{array}\right)=\left(\begin{array}{l}
b_{1} \\
b_{2} \\
b_{3} \\
b_{4}
\end{array}\right)
$$

At $\mathbf{x}^{n}=\left(x_{1}^{n}, x_{2}^{n}, x_{3}^{n}, x_{4}^{n}\right)^{T}$, we have

$$
\left(\begin{array}{cccc}
\beta_{1}+2 \frac{\beta_{2}}{h^{2}} & 0 & 0 & 0 \\
0 & \beta_{1}+2 \frac{\beta_{2}}{h^{2}} & 0 & 0 \\
0 & 0 & \beta_{1}+2 \frac{\beta_{2}}{h^{2}} & 0 \\
0 & 0 & 0 & \beta_{1}+2 \frac{\beta_{2}}{h^{2}}
\end{array}\right)\left(\begin{array}{c}
x_{1}^{n+1} \\
x_{2}^{n+1} \\
x_{3}^{n+1} \\
x_{4}^{n+1}
\end{array}\right)=\left(\begin{array}{c}
b_{1}+\frac{\beta_{2}}{h^{2}} x_{2}^{n} \\
b_{2}+\frac{\beta_{2}}{h^{2}}\left(x_{1}^{n}+x_{3}^{n}\right) \\
b_{3}+\frac{\beta_{2}}{h^{2}}\left(x_{2}^{n}+x_{4}^{n}\right) \\
b_{4}+\frac{\beta_{2}}{h^{2}} x_{3}^{n}
\end{array}\right) .
$$

Since $\beta_{1}>0$, the coefficient matrix $\mathbf{A}$ in (6.3) is strictly diagonally dominant and thus the Jacobi method converges for any mesh size $h>0$. Moreover, at iterate $\mathbf{x}^{n}=\left(x_{1}^{n}, x_{2}^{n}, \ldots, x_{i}^{n}, \ldots, x_{5}^{n}\right)^{T}$, since $\beta_{1}+2 \beta_{2} / h^{2}>0$, the iterate $x_{i}^{n+1}$ is a positive weighted average of $\mathbf{x}^{n}$ at its neighbours $i-1$ and $i+1$. Therefore, starting from any vector $\mathbf{b}$ and any initial guess $\mathbf{x}^{0}$, the converged solution $\mathbf{x}$ will be a smooth quantity depending on $\mathbf{b}$. This is the smoothing effect, and it indicates that the smoothed search direction of the control update $\delta \mathbf{g}^{n}$ in (6.2) is smoother and has a higher regularity than the plain Steepest Descent direction $-\left(\tau+\beta_{1} \mathbf{g}^{n}-\beta_{2} \Delta \mathbf{g}^{n}\right)$. The computational cost of the smoothing process consists of the one-time Finite Element assembly of the surface mass and surface Laplacian matrices and the solution of the Laplacian-like linear system (6.2) at each nonlinear optimization iteration. Finally, we note that a different argument for showing the smoothing effect of the operator $\left(\beta_{1} \mathbf{I}+\beta_{2} \Delta\right)^{-1}$ can be derived based on its Green's function, and the numerical smoothing device above can be viewed as solving the gradient equation (6.1) via a Preconditioned Richardson method. Alternatively, (6.2) can be viewed as the realization of the $\mathbf{H}^{1}$ norm of the reduced gradient in the function space [70]. Finally, we note that when $\beta_{2}>0$ is enforced, the numerical smoothing effect would be automatically incorporated in the Newton iteration of the coupled KKT system in the fullspace approach. Taking the Gauss-Newton method $[19,28,104]$ as an example, the inverse of the $(\delta \mathbf{g}, \delta \mathbf{g})$ block in the Gauss-Newton iteration has the same form as the smoothing operator in (6.2). 


\section{CHAPTER 6. NUMERICAL OPTIMIZATION ALGORITHMS}

In the literature of aerodynamic shape optimization [83-85], the Jameson-type smoothing, which is based on introducing a specific weighted Sobolev norm, has been tested extensively. It directly applies a similar smoothing operator to the Steepest Descent direction without any modification to the objective function. However, for the control model of the CHT process, it turns out that adding the regularization term on $\nabla \mathbf{g}$ to the objective function (2.4) is necessary. If we apply the smoothing to the reduced gradient without the penalty term on $\nabla \mathbf{g}$ in the objective function, we can only generate a smooth search direction for the control, but still with tiny line search steps, which hinders the rate of convergence and makes the method impractical. This is due to the lack of the mathematical justification of using the smoothing operator, since the line search routine is not able to find a feasible stepsize along the smooth, but modified search direction for the unmodified objective function. In other words, the numerical optimization algorithm is not consistent with the discrete optimization problem in this case. Section 7.2.1 is devoted to further illustrate this point via numerical experiments. Finally, we summarize the smoothed Steepest Descent method in Algorithm 6.3.2.

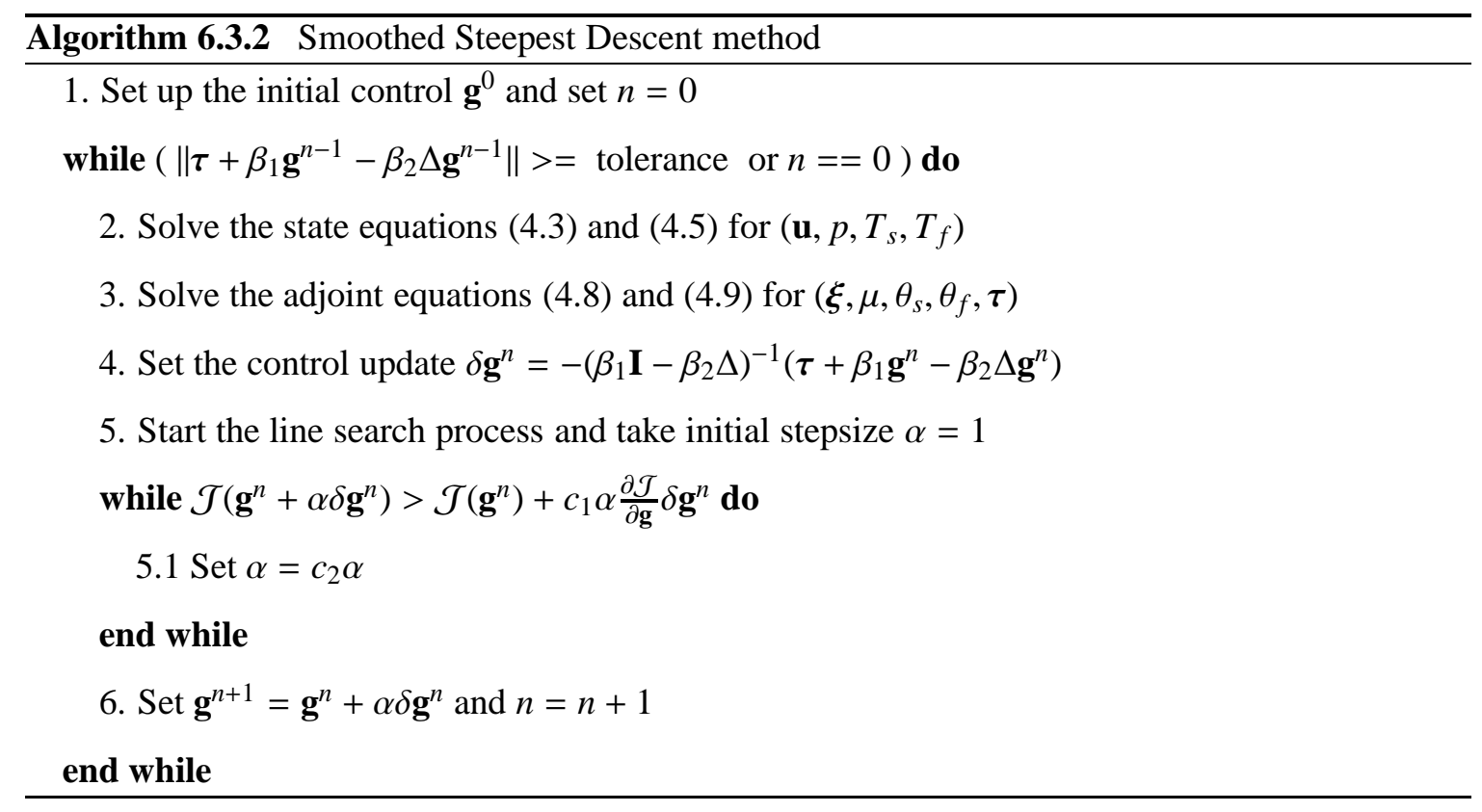




\subsection{Limited-Memory BFGS Method}

The Steepest Descent method is equivalent to taking the Hessian as the identity matrix at each nonlinear optimization iteration, namely, $\mathbf{B} \equiv \mathbf{I}$ in the Newton equation $\mathbf{B} \delta \mathbf{g}=-\partial \mathcal{J} / \partial \mathbf{g}$. In comparison, the smoothed Steepest Descent method takes the constant Hessian $\mathbf{B} \equiv \beta_{1} \mathbf{I}-\beta_{2} \Delta$ at each iteration. Both of these methods are limited to linear convergence rate due to the lack of updates on the curvature information along the nonlinear optimization iterations. Quasi-Newton methods, such as the BFGS method, use successive gradient information from previous iterations to build up a local Hessian approximation $\mathbf{B}^{k}$ at the iteration $k$. With an efficient line search procedure, BFGS methods have a superlinear convergence rate [104].

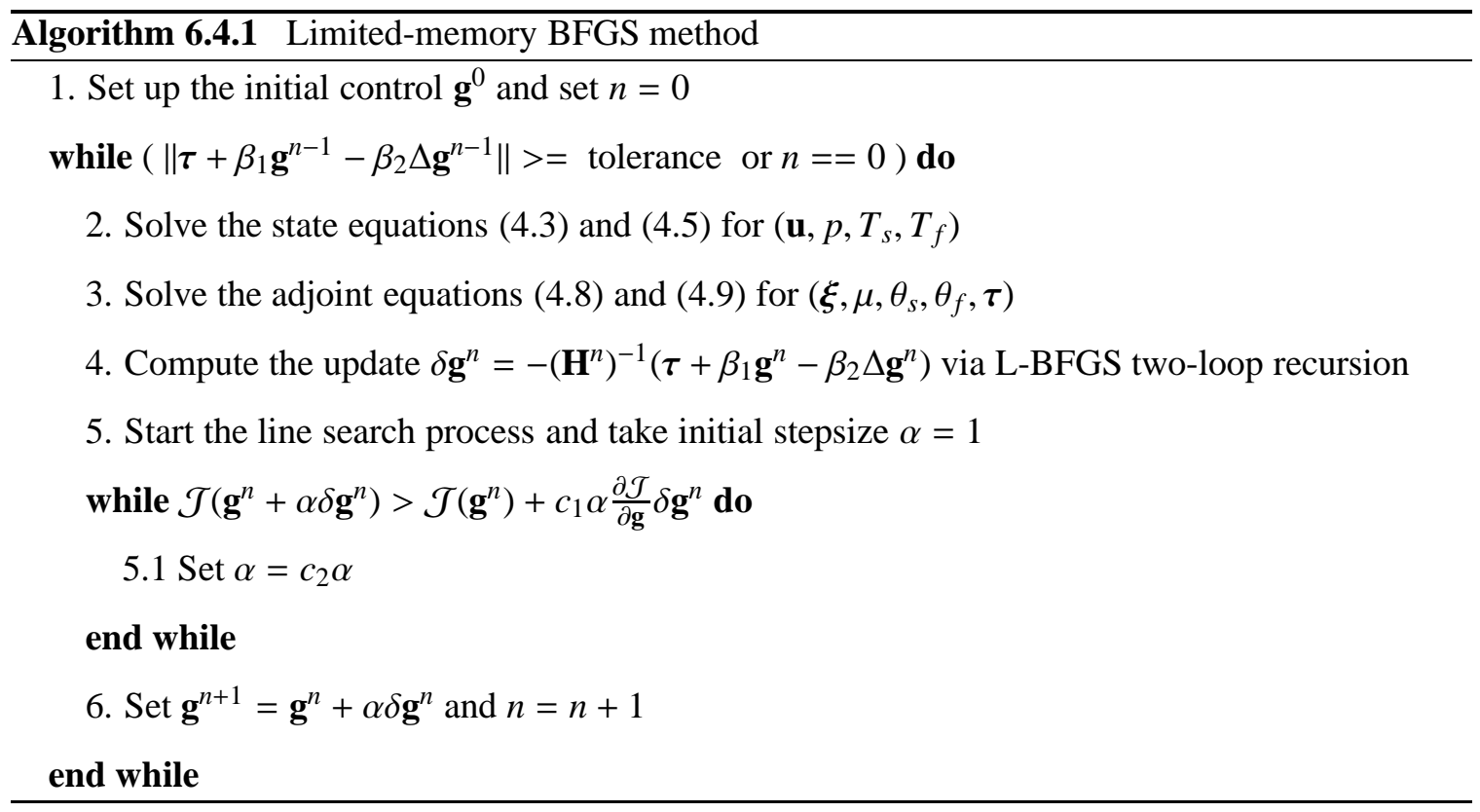

We denote the inverse of Hessian approximation as,

$$
\mathbf{H}^{k}=\left(\mathbf{B}^{k}\right)^{-1},
$$


then the BFGS updating formula [104] for the objective function $\mathcal{J}$ with gradient $\frac{\partial \mathcal{J}}{\partial \mathbf{g}}$ is,

$$
\begin{aligned}
& s^{k}=\mathbf{g}^{k+1}-\mathbf{g}^{k} ; \\
& y^{k}=\frac{\partial \mathcal{J}}{\partial \mathbf{g}}\left(\mathbf{g}^{k+1}\right)-\frac{\partial \mathcal{J}}{\partial \mathbf{g}}\left(\mathbf{g}^{k}\right) ; \\
& \rho^{k}=\frac{1}{\left(y^{k}\right)^{T} s^{k}} ; \\
& V^{k}=\mathbf{I}-\rho^{k} y^{k}\left(s^{k}\right)^{T} ; \\
& \mathbf{H}^{k+1}=\left(V^{k}\right)^{T} \mathbf{H}^{k} V^{k}+\rho^{k} s^{k}\left(s^{k}\right)^{T} .
\end{aligned}
$$

In practice, what is needed is the search direction,

$$
\delta \mathbf{g}=-\mathbf{B}^{-1} \frac{\partial \mathcal{J}}{\partial \mathbf{g}}=-\mathbf{H} \frac{\partial \mathcal{J}}{\partial \mathbf{g}}
$$

which is a matrix-vector multiplication. The storage and the manipulation of the dense inverse Hessian approximation $\mathbf{H}^{k+1}$ can be avoided by storing a sequence of vector pairs $\left\{s^{i}, y^{i}\right\}$, where $i=1,2, \ldots, k$, at each nonlinear optimization iteration $k$. Moreover, when $k$ is large, in order to reduce the memory occupied by $\left\{s^{i}, y^{i}\right\}$, only the most recent $m$ pairs of $\left\{s^{i}, y^{i}\right\}$ are stored, namely, $i=k-m+1, \ldots, k$. Furthermore, we note that $m=20$ or 30 is sufficient for the control problems in this study, as shown in Table 7.5 in Section 7.3.1. The resulting algorithm is the Limited-memory BFGS method $[98,102,104]$ with the formula,

$$
\begin{aligned}
\mathbf{H}^{k+1}= & \left(\left(V^{k}\right)^{T} \ldots\left(V^{k-m+1}\right)^{T}\right) \mathbf{H}_{0}^{k}\left(V^{k-m+1} \ldots V^{k}\right) \\
& +\rho^{k-m+1}\left(\left(V^{k}\right)^{T} \ldots\left(V^{k-m+1}\right)^{T}\right) s^{k-m+1}\left(s^{k-m+1}\right)^{T}\left(V^{k-m+1} \ldots V^{k}\right) \\
& +\rho^{k-m+2}\left(\left(V^{k}\right)^{T} \ldots\left(V^{k-m+2}\right)^{T}\right) s^{k-m+2}\left(s^{k-m+2}\right)^{T}\left(V^{k-m+2} \ldots V^{k}\right) \\
& +\ldots \\
& +\rho^{k} s^{k}\left(s^{k}\right)^{T}
\end{aligned}
$$

where $s^{k}, y^{k}$ and $V^{k}$ are defined in (6.6).

In our implementation, we have set, at the $k$-th nonlinear iteration, the initial inverse Hessian $\mathbf{H}_{0}^{k}:=\left(\beta_{1} \mathbf{I}-\beta_{2} \Delta\right)^{-1}$, which is the smoothing operator in Section 6.3. Therefore, it is clear that in (6.8) the same smoothing procedure has been carried out explicitly in the middle step of the L-BFGS two-loop recursion at each nonlinear optimization iteraion $k$. The L-BFGS algorithm for 
the boundary control of the CHT process and the L-BFGS two-loop recursion implementation of (6.8) are summarized in Algorithm 6.4.1 and Algorithm 6.4.2 [102, 104]. Our implementation on the two-loop recursion follows [72,104].

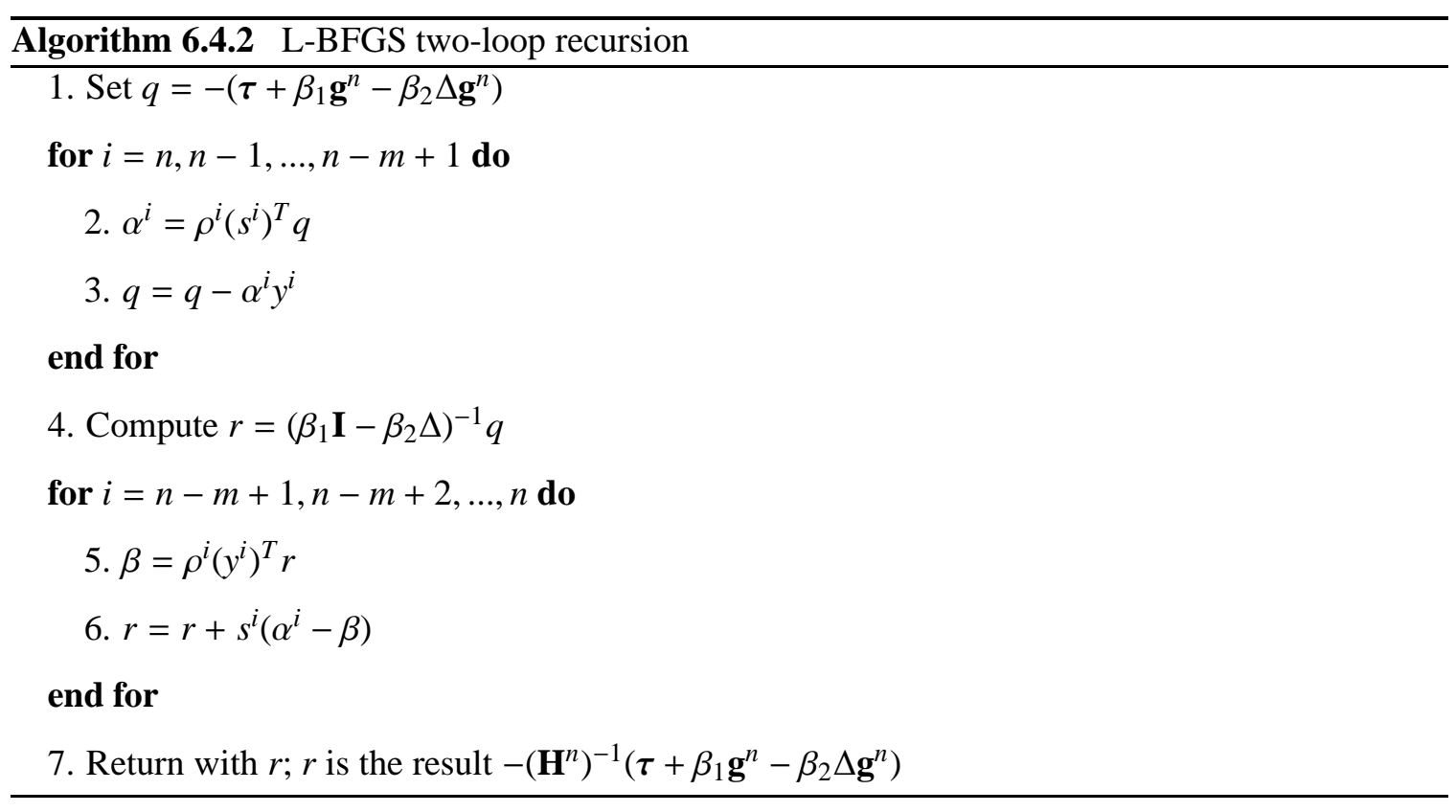

\subsection{Numerical Continuation on Regularization Parameters}

For highly nonlinear optimization problems, such as the boundary control of systems governed by multiphysics processes, it is essential to have an initial guess close to the exact solution in order to make the optimization scheme converge to the correct minimizer. And it is also essential to apply regularization to improve the convexity of the objective function and the regularity of the boundary control solution. Indeed, as shown in Section 7.2.1, even for a simple test problem of recovering the boundary control profile of Poiseuille flow, regularization is required. Furthermore, the situation is most challenging when the initial guess is far from optimal. In that case, we need not only bring the iterate close to optimal solution, but also adjust the right amount of regularization and keep the balance between the dominance of the observation term and the efficiency of the penalty terms in (2.4). More specifically, with too much regularization, the optimization schemes may converge 


\section{CHAPTER 6. NUMERICAL OPTIMIZATION ALGORITHMS}

prematurely to a suboptimal control profile and, on the other hand, with insuffcient regularization the optimization schemes may fail to converge at all. In Section 7.3, we demonstrate an example of such an situation.

Our solution strategy for problems with an initial guess of low quality is to apply the numerical continuation to the regularization parameters $\beta_{1}$ and $\beta_{2}$ in (2.4). Numerical continuation is a standard globalization technique in solving nonlinear equations. It is quite useful when the target problem can be parameterized on a small set of continuation parameters which characterize the difficulty of the underlying physics. Some examples of such parameters are the Reynolds number [26], the pseudotimestep in steady state problems [35,90], the mesh size [120], and the barrier parameter in nonlinear optimization [128]. The numerical continuation process always starts from solving a much easier subproblem, namely, a subproblem with a lower Reynolds number, a smaller timestep, a coarser mesh, or a large barrier parameter. It then incrementally changes the set of continuation parameters and solves a sequence of progressively more difficult subproblems. With increasingly better initial guesses from solving each of the subproblems, we finally obtain the solution of the original difficult problem.

When applying the regularization continuation technique to the boundary control of CHT processes, with an initial control profile that is far from optimal, we would take a relatively large amount of regularization, namely, $\beta_{1}$ and $\beta_{2}$ that are an order of magnitude larger than what would be used in the ideal case, to formulate an easier subproblem A. See (7.2) and Section 7.2.4 for details. This allows the optimization schemes to identify an improved suboptimal control profile. Then, we slightly reduce the regularization parameters $\beta_{1}$ and $\beta_{2}$ to formulate another subproblem $\mathrm{B}$, which is more difficult than the subproblem A but easier than the original problem. With the newly identified suboptimal control profile from the subproblem A, the optimization schemes identify a further improved suboptimal control profile from the subproblem B. We repeat this process, until either the maximum allowed continuation steps or the tolerance of the objective function value has been reached. We note that each time we reduce $\beta_{1}$ and $\beta_{2}$, we have modified and, actually, reduced the objective function. This modification generally increases the reduced gradient, because the computed optimal control of the previous subproblem is not optimal in the current subproblem. 
We summarize the L-BFGS method with $k$-phase regularization continuation in Algorithm 6.5.1.

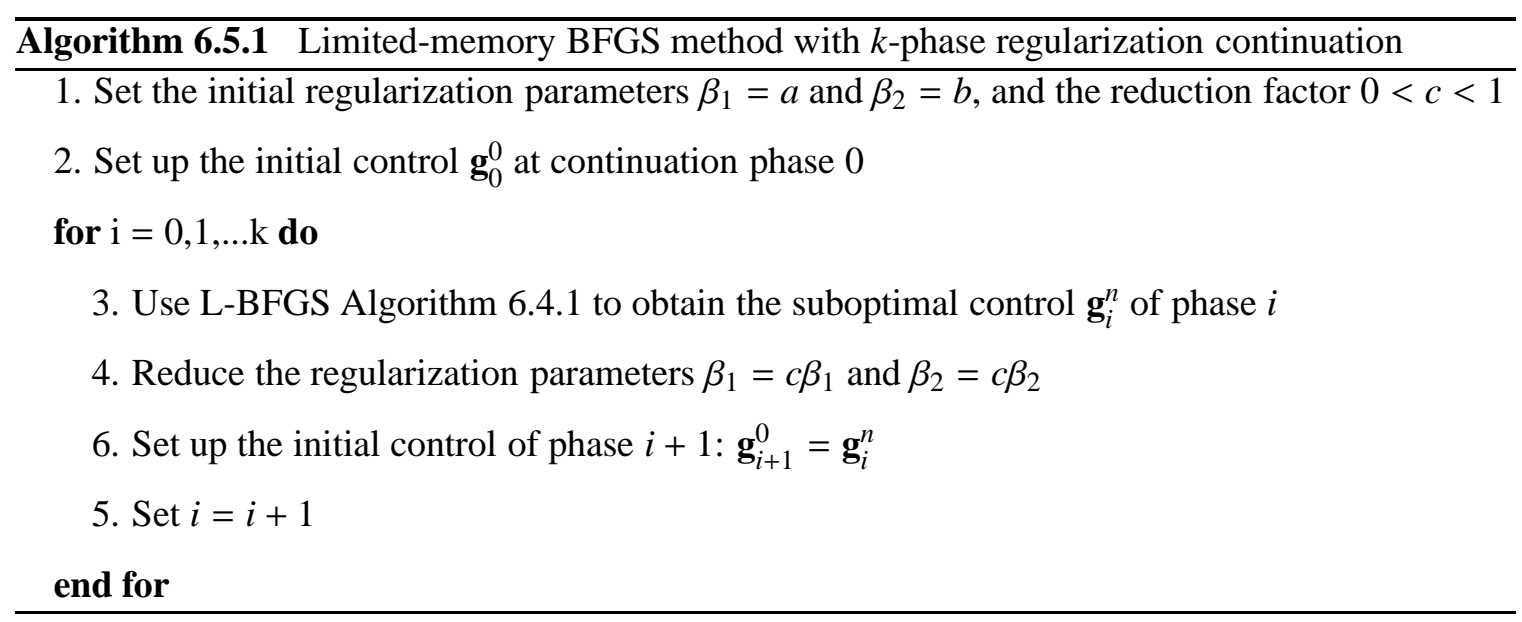




\section{Chapter 7}

\section{Numerical Test Models for Smooth and Robust Solutions}

\subsection{Overview}

We study three numerical test problems for the Dirichlet boundary control of CHT processes. For the first test problem in Section 7.2, we use three different numerical schemes, namely, the plain Steepest Descent method, the smoothed Steepest Descent method and the L-BFGS method, to recover the boundary control profile of a Poiseuille flow. Moreover, we study the effect of regularization and the necessity of keeping $\beta_{2}>0$ on both the continuous and the discrete levels in Section 7.2.1, the smoothing operator in Section 7.2.2 and the comparisons between the smoothed Steepest Descent method and the L-BFGS method in Section 7.2.3. Furthermore, we discuss various observation operators and the strategy for choosing appropriate regularization parameters in Section 7.2.4.

For the test problems in Section 7.3, we solve the boundary control problem for the channel flow again and then for the flow past a square. In the channel flow case, the target temperature $T_{d}$ is generated by the velocity control profile of two adjacent Poiseuille-flow peaks pointing to the $x$ direction. Since we start from an initial control profile that is far from optimal and the appropriate regularization parameters are hard to determine, in Section 7.3.1, we use this test to demonstrate the robustness of the L-BFGS method with 5-phase regularization continuation. We also show that, 
when the L-BFGS method is embedded in the regularization continuation loop, oversolving can be avoided by choosing a relaxed relative tolerance for the L-BFGS loop. In Section 7.3.2, we apply the optimization schemes developed in previous sections to resolve the boundary control problem of the flow past a square cylinder. This involves a more complex geometry and fluid velocity field and further demonstrates the effectiveness and robustness of our optimization scheme.

\subsection{Numerical Test Problem 1}

We set up the test model with the following data: $R e=1000, k_{s}=1$ and $k_{f}=0.1$, where the Reynolds number $R e:=1 / v . \quad v$ is the kinematic viscosity. This model is modified from a test case used for the temperature boundary control of convection-diffusion equations in [63]. The computational domain consists of $\Omega_{2}=[0,1] \times[0,0.75]$ and $\Omega_{1}=[0,1] \times[0.75,1]$. We take the heating source in the solid domain $\Omega_{1}$ as a constant value $q=6$, and the initial control $\mathbf{g}$ in (2.1) is set to be the Poiseuille flow profile $\left(1.5 y-2 y^{2}, 0\right)^{T}$ on $\Gamma_{i}=\{0\} \times[0,0.75]$. The exact optimal control is given by

$$
\mathbf{g}=2\left(1.5 y-2 y^{2}, 0\right)^{T}
$$

The target temperature $T_{d}$ in (2.4) is calculated using the fluid velocity induced by the optimal control profile in (7.1) and is shown in Figure 7.1. To assemble the objective function (2.4), we set the regularization parameters as follows: $\beta_{1}=1$ and $\beta_{2}=1$ when the observation domain $\Omega_{d}=\Omega_{2}$ in (2.4); $\beta_{1}=5 \times 10^{-2}$ and $\beta_{2}=5 \times 10^{-3}$ when $\Omega_{d}=[0.075,1] \times\{0.75\}$. In the following discussion, if not specified, we assume $\Omega_{d}=\Omega_{2}$. The IFISS package [47,117] has been used for the finite element assembly. In the mesh generation, the fluid-solid domain is discretized with 40 Q2 elements in both $x$ and $y$ directions. In a single Q2 cell, there are 9 degrees of freedom (DOF) for each component of the triple $\left(u_{x}, u_{y}, T\right)$. The fluid pressure is discretized with $40 \mathrm{Q} 1$ elements in $x$ and 30 in $y$ direction on the fluid domain $\Omega_{2}$. Each Q1 cell has 4 DOF. 

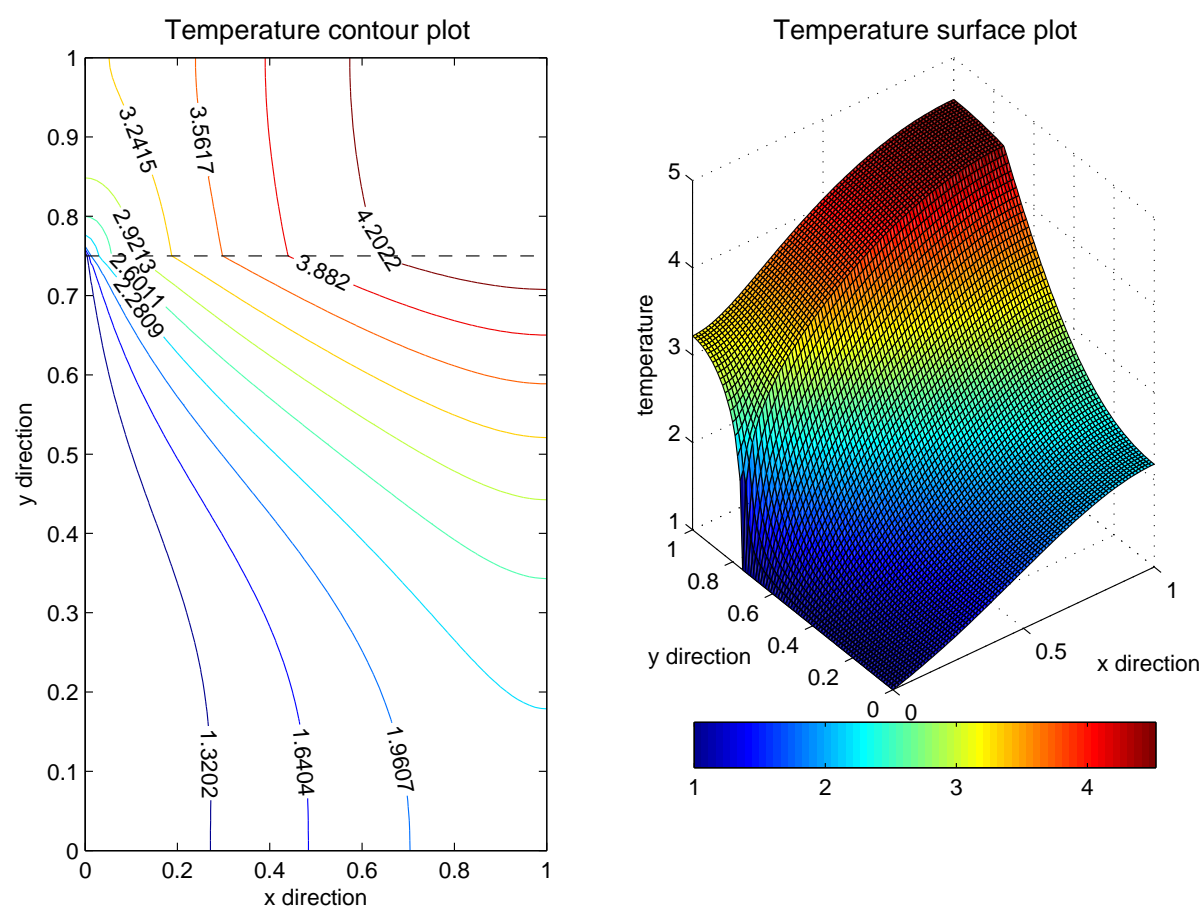

Figure 7.1: Target temperature distribution with prescribed inflow $\mathbf{g}=2\left(1.5 y-2 y^{2}, 0\right)^{T}$. The dashed line indicates the fluid-solid interface. 


\subsubsection{Necessity of the Regularization Term on the Control Gradient}

In Section 5.3, we have emphasized the importance of keeping $\beta_{2}>0$ in (2.4) on both the continuous and the discrete levels to ensure smooth controls. When $\beta_{2}=0$ in the discrete objective function, only nonsmooth control profiles can be generated. We display the nonsmooth flow solution after 100 plain Steepest Descent iterations in Figure 7.2. Moreover, the situation becomes clearer when we use $\beta_{2}=0$ in the discrete objective function on different meshes. We show in Figure 7.3 the computed control profiles on a sequence of meshes. It is evident that two patterns exist, namely, one for $h=1 / 24,1 / 40,1 / 60$ and the other one for the rest, and both of them become increasingly oscillatory as the meshsize $h$ decreases. This is caused by the inconsistency between the continuous control problem, in which $\beta_{2}>0$, and the discrete control problem, in which $\beta_{2}=0$. Furthermore, in Table 7.1, we see that the computed optimal objective function values do not have a limit point as the meshsize $h$ decreases, and this renders the solution process of the discrete optimal control problem unsuccessful and allows only nonsmooth, nonunique, and mesh-dependent local minima. Therefore, $\beta_{2}>0$ on both the continuous and the discrete levels is crucial for obtaining smooth, unique and mesh-independent global minimum. Moreover, it is clear from Table 7.2 that when $\beta_{2}>$ 0 and the numerical smoothing operator is incorporated in the optimization scheme, the computed optimal objective function values converge as the meshsize $\mathrm{h}$ decreases.

In fact, the regularization term $\frac{\beta_{2}}{2} \int_{\Gamma_{i}}|\nabla \mathbf{g}|^{2}$ in (2.4) not only penalizes the objective function for the nonsmoothness of the control $\mathbf{g}$, but it justifies the mathematical derivation of the smoothing operator in the smoothed Steepest Descent method in Section 6.3 and the L-BFGS method in Section 6.4. When $\beta_{2}=0$, in the smoothed Steepest Descent method, if we still use $\left(\beta_{1} \mathbf{I}-1 \cdot \Delta\right)^{-1}$ as the smoothing operator on the reduced gradient, the method will stagnate after 100 optimization iterations, but with a smooth flow solution. There is no mathematical justification for using the operator $\beta_{1} \mathbf{I}-1 \cdot \Delta$ as an approximation for the Hessian of the objective function, and, in fact, the reduced gradient ceases to decrease. When $\beta_{2}=0$ and with the smoothing operator turned on, in the the L-BFGS method, the line search process of the first few optimization iterations fails quickly. Again, this is no mathematical justification for using the smoothing operator, since the line search routine is not able to find a feasible stepsize along the smooth, but modified search direction for the 
unmodified objective function. In the following discussion, we always use $\beta_{2}>0$.
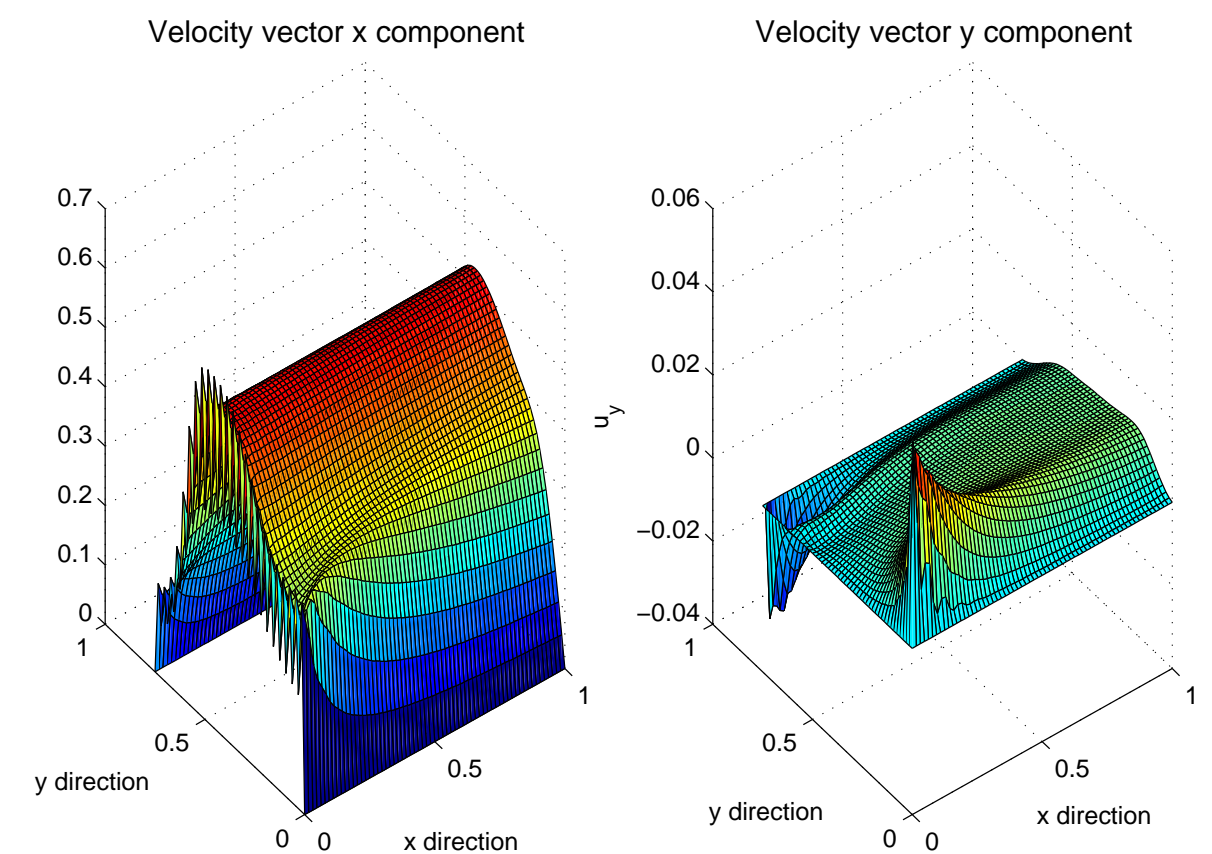

Figure 7.2: Nonsmooth velocity solution after 100 plain Steepest Descent iterations when without smoothness control, or when $\beta_{2}=0$.

\subsubsection{Necessity of the Smoothing Operator for the Steepest Descent Method}

In order to show the necessity of the smoothing operator for the Steepest Descent method, we compare the performance of the plain Steepest Descent method with that of the smoothed Steepest Descent method for the test problem 1. We set $\beta_{1}=\beta_{2}=1$ and $\Omega_{d}=\Omega_{2}$ in (2.4). After 100 nonlinear optimization iterations with more than 600 line search iterations, the Steepest Descent method is not able to converge. The nonsmoothness of the reduced gradient (or control update) forces the line search iteration to take tiny steps at every nonlinear optimization iteration. Eventually, the Steepest Descent method stagnates. In contrast, after 32 nonlinear optimization iterations with 101 Armijo line search iterations, the smoothed Steepest Descent method converges. Moreover, this method is able to obtain totally smooth control updates, which allow the line search process to take 

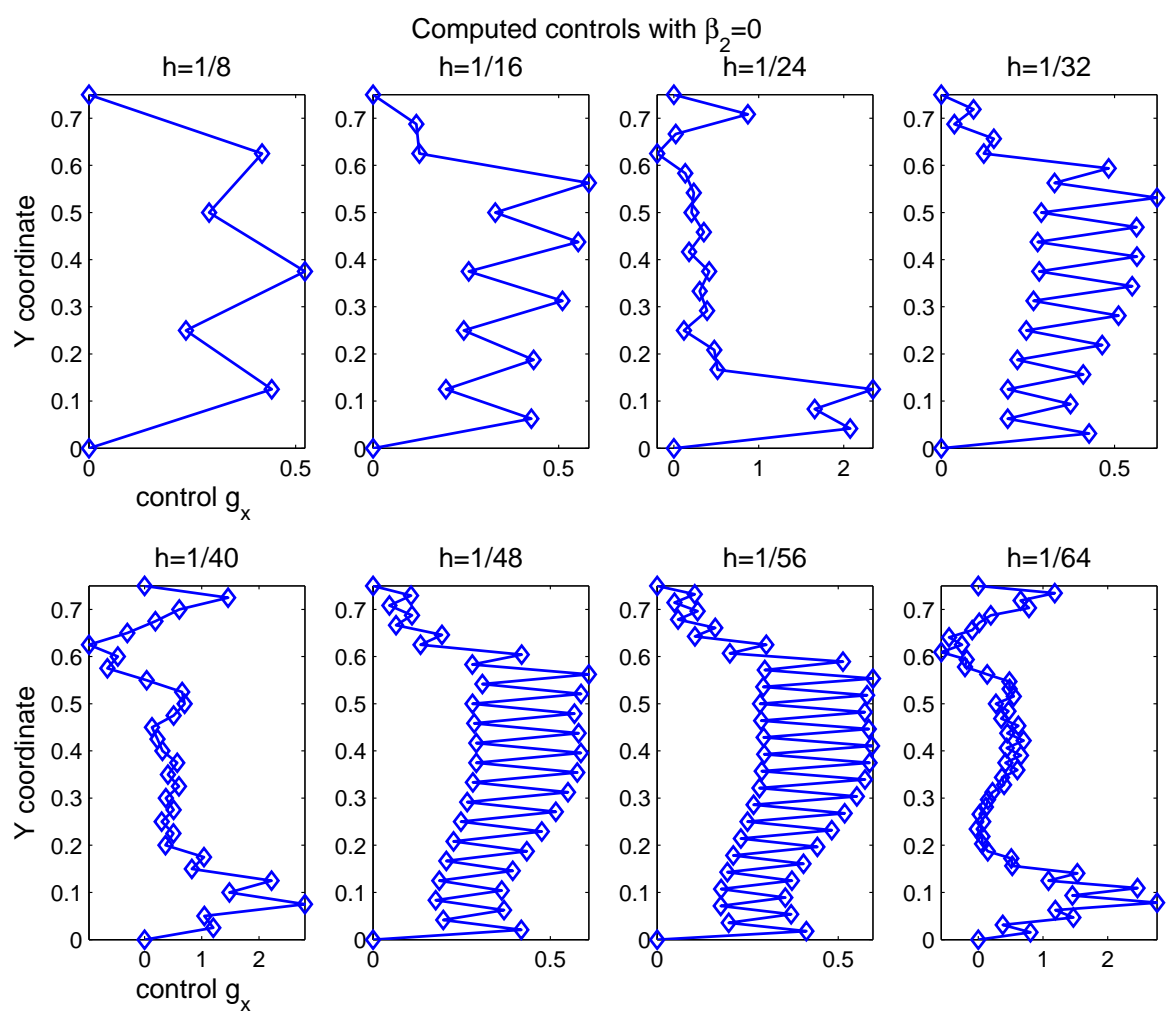

Figure 7.3: Nonsmooth control profiles generated without smoothness control, using $\beta_{2}=0$. There are two patterns: one for $h=1 / 24,1 / 40,1 / 60$ and the other one for the rest, and both are increasingly oscillatory as meshsize $h$ decreases.

\begin{tabular}{ccccccccc}
\hline \multicolumn{8}{c}{ Meshsize } & $h \longrightarrow 0$ \\
\hline & $1 / 8$ & $1 / 16$ & $1 / 24$ & $1 / 32$ & $1 / 40$ & $1 / 48$ & $1 / 56$ & $1 / 64$ \\
\hline $\mathcal{J}\left(\mathbf{g}_{h}^{\star}\right)$ & 0.0514 & 0.0507 & 0.7175 & 0.0510 & 1.087 & 0.0515 & 0.0517 & 0.8331 \\
\hline
\end{tabular}

Table 7.1: Computed optimal objective function values using $\beta_{2}=0$. The comparison is made for different meshsizes. It is evident that the objective function values $\mathcal{J}\left(\mathbf{g}_{h}^{\star}\right)$ do not have a limit point, as the meshsize $h$ goes to 0 . This shows the need for the smoothing term in the objective function. 


\begin{tabular}{|c|c|c|c|c|c|c|c|c|}
\hline & \multicolumn{8}{|c|}{ Meshsize $\quad h \longrightarrow 0$} \\
\hline & $1 / 8$ & $1 / 16$ & $1 / 24$ & $1 / 32$ & $1 / 40$ & $1 / 48$ & $1 / 56$ & $1 / 64$ \\
\hline $\mathcal{J}\left(\mathbf{g}_{h}^{\star}\right)$ & 0.6941 & 0.8703 & 0.9540 & 1.003 & 1.035 & 1.057 & 1.074 & 1.087 \\
\hline
\end{tabular}

Table 7.2: Computed optimal objective function values using $\beta_{2}=1$ and the L-BFGS method with numerical smoothing. The comparison is made for different meshsizes. It is evident that the objective function values $\mathcal{J}\left(\mathbf{g}_{h}^{\star}\right)$ converge, as the meshsize $h$ goes to 0 . This demonstrate the effectiveness of the smoothing term in the objective function and the numerical smoothing operator in the optimization scheme.

much larger steps. In conclusion, the smoothing operator $\left(\beta_{1} \mathbf{I}-\beta_{2} \Delta\right)^{-1}$ is essential for the Steepest Descent method to converge to the optimal boundary control.

\subsubsection{Comparison between the Smoothed Steepest Descent Method and the L-BFGS Method}

We note that both the smoothed Steepest Descent method and the L-BFGS method have converged successfully for the test problem 1 and obtained control profiles of the same quality on different meshsizes. In Table 7.3, we show the order of convergence of the computed control profiles $g_{h}^{\star}$ towards the exact optimal control $g^{\star}$ of (7.1). It is evident that both methods have almost the same error and order of convergence. Therefore, their efficiency is determined by their corresponding counts of nonlinear optimization iterations and line search iterations on a fixed mesh. For a mesh with $40 \times 40$ Q2 cells on $\Omega$ and the nonlinear tolerance $t o l=10^{-6}$, the smoothed Steepest Descent method takes 32 optimization iterations with 101 Armijo line search iterations to converge to the optimal boundary control. In contrast, the L-BFGS method only takes 9 optimization iterations and 13 Armijo line search iterations to converge. In fact, each line search iteration is computationally quite expensive, since for each candidate stepsize we have to recompute the objective function value, which requires the solution of the state equations. Therefore, a large number of line search iterations taken by the smoothed Steepest Descent method indicates that this method is very expensive. In 
Figure 7.4, the history of the reduced gradient confirms the superlinear convergence of the L-BFGS method. As shown in Figure 7.5, the computed optimal control $\mathbf{g}$ can be seen from the flow solution at $x=0$ during the last L-BFGS line search iteration. The computed temperature distribution is shown in Figure 7.6, which is quite close to the target temperature profile in Figure 7.1. Figure 7.7 and Figure 7.8 contain the adjoint temperature solution and adjoint velocity solution at the last L-BFGS line search iteration, respectively. The initial control, the computed optimal controls and the exact optimal control are shown in Figure 7.11.

\begin{tabular}{ccccc}
\hline & \multicolumn{2}{c}{ Smoothed SD } & \multicolumn{2}{c}{ L-BFGS } \\
\hline Meshsize $h$ & $\left\|g_{h}^{\star}-g^{\star}\right\|_{\mathbf{L}^{2}\left(\Gamma_{i}\right)}$ & OC & $\left\|g_{h}^{\star}-g^{\star}\right\|_{\mathbf{L}^{2}\left(\Gamma_{i}\right)}$ & OC \\
\hline $1 / 8$ & $4.6345 \times 10^{-2}$ & - & $4.6345 \times 10^{-2}$ & - \\
$1 / 16$ & $2.1662 \times 10^{-2}$ & 1.10 & $2.1662 \times 10^{-2}$ & 1.10 \\
$1 / 32$ & $9.1681 \times 10^{-3}$ & 1.24 & $9.1682 \times 10^{-3}$ & 1.24 \\
$1 / 64$ & $3.6426 \times 10^{-3}$ & 1.33 & $3.6426 \times 10^{-3}$ & 1.33 \\
\hline
\end{tabular}

Table 7.3: Order of convergence (OC) for the smoothed Steepest Descent method and the L-BFGS method. Both methods are effective and have the same OC when the numerical smoothing device is turned on.

\subsubsection{Choices of the observation operator and regularization parameters}

In the numerical experiments above, we have defined the observation operator in the objective function (2.4) to be the whole fluid region, namely, $\Omega_{d}=\Omega_{2}$. For some applications, such as the cooling process in power plants, the fluid-solid interfacial temperature is the quantity of interest, since it serves as an indicator of the solid-fuel failure [130]. Therefore, we have carried out an experiment using $\Omega_{d}=[0.075,1] \times\{0.75\}$ as the observation domain. The same interfacial observation domain has been used in temperature boundary control problems for convection-diffusion equations [63,116]. We set $\beta_{1}=5 \times 10^{-2}$ and $\beta_{2}=5 \times 10^{-3}$ and keep all other previous numerical parameters intact. The observation domain $\Omega_{d}=[0.075,1] \times\{0.75\}$ is a line segment and has a 


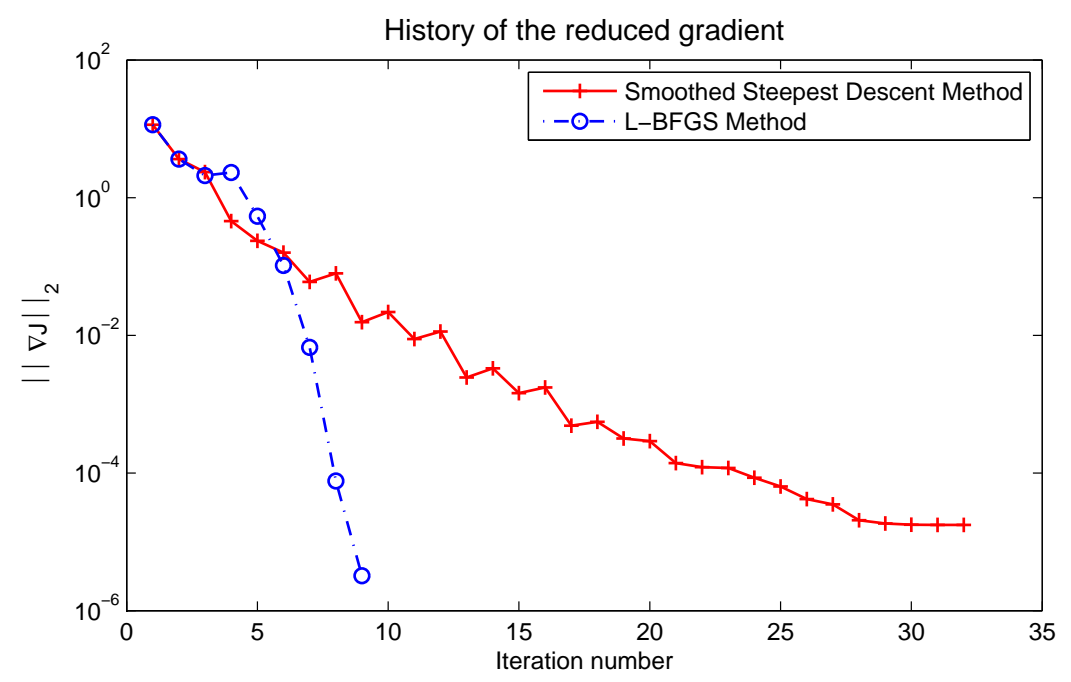

Figure 7.4: Convergence history of the smoothed Steepest Descent method and the L-BFGS methods.
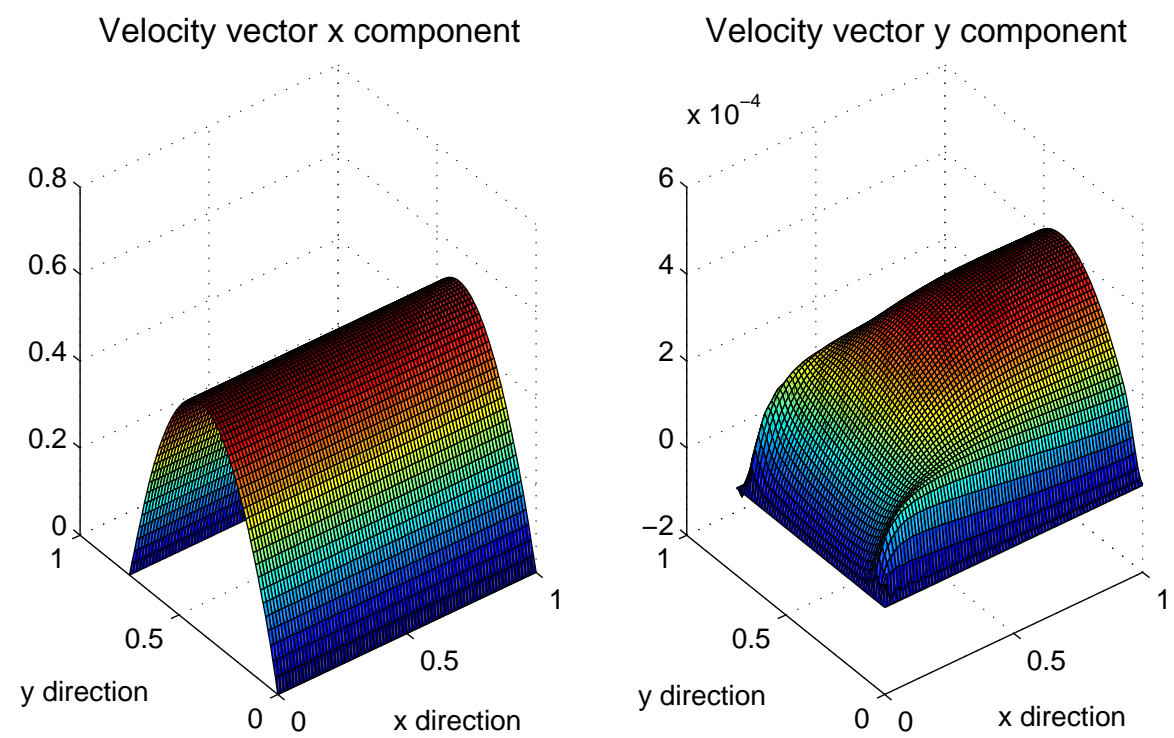

Figure 7.5: Fluid velocity solution at the last L-BFGS line search iteration. 

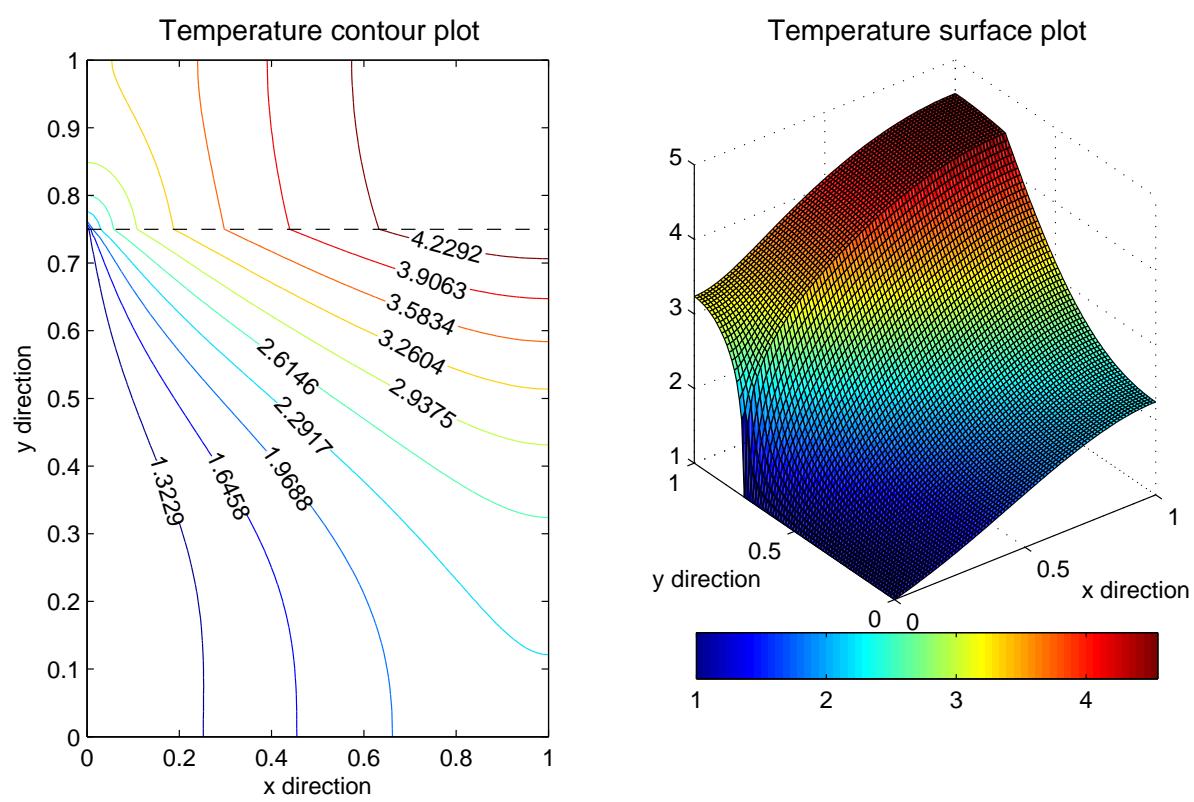

Figure 7.6: Temperature solution at the last L-BFGS line search iteration with $\Omega_{d}=\Omega_{2}$.
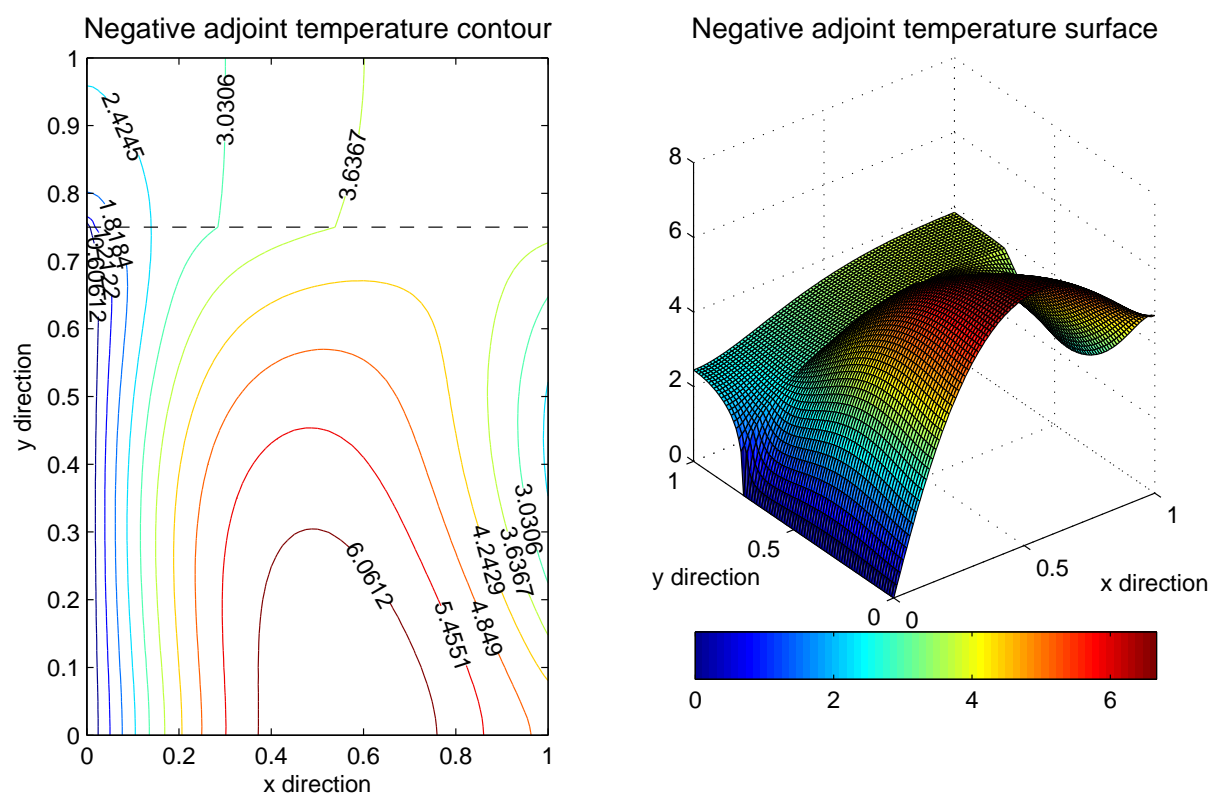

Figure 7.7: Last adjoint temperature solution of the L-BFGS method with $\Omega_{d}=\Omega_{2}$. 

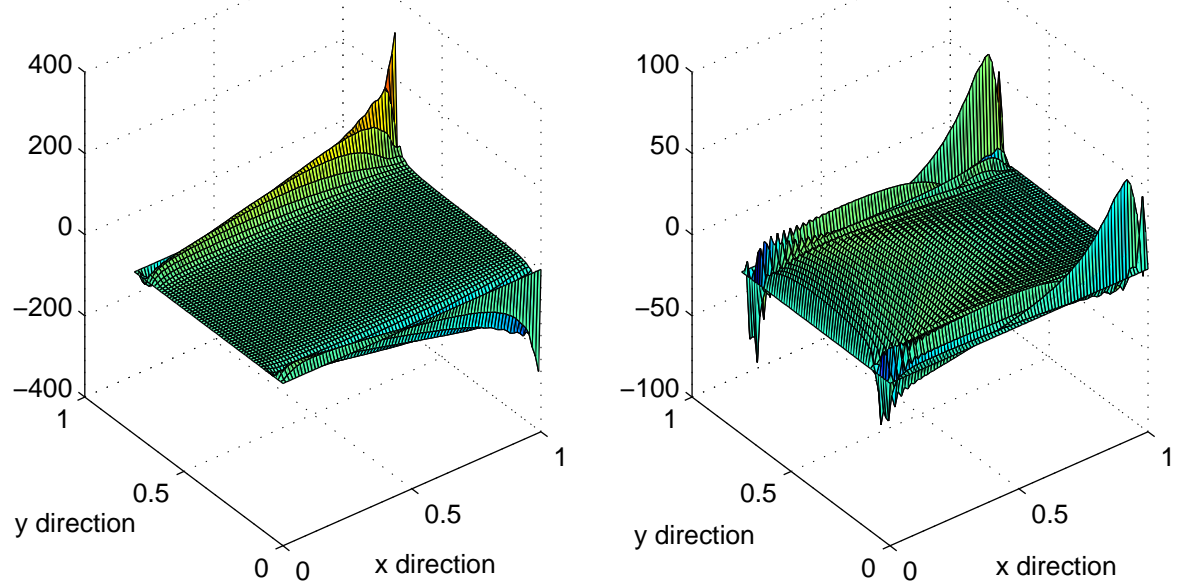

Figure 7.8: Last adjoint velocity solution of the L-BFGS method with $\Omega_{d}=\Omega_{2}$.

much smaller measure than that of the previous one $\Omega_{d}=\Omega_{2}$; therefore, to make the observation term in (2.4) dominant and thus find an accurate optimal control, we have to reduce $\beta_{1}$ and $\beta_{2}$ to a smaller amount of regularization. Moreover, since the gradient of the temperature difference $\frac{1}{2} \int_{\Omega_{d}}\left(T-T_{d}\right)^{2} d x$ in (2.4) is the forcing term in the adjoint equations (4.9) and has a much smaller magnitude when $\Omega_{d}=[0.075,1] \times\{0.75\}$, the corresponding adjoint quantities have a smaller magnitude as well. This is confirmed in Figure 7.9 and Figure 7.10.

With the parameters above, both the smoothed Steepest Descent method and L-BFGS method have converged for the control problem with $\Omega_{d}=[0.075,1] \times\{0.75\}$. The smoothed Steepest Descent method takes 36 nonlinear optimization iterations with 101 line search iterations to converge, while the L-BFGS takes only 8 nonlinear optimization iterations with 4 line search iterations. In Figure 7.11, we have shown the computed controls from both methods for two different observation operators. It is clear that when the observation operator involves more points, namely, $\Omega_{d}=\Omega_{2}$, the numerical approximations of the optimal control are closer to the exact optimal control.

Choice of the regularization parameters affects the robustness of the optimization scheme. In this study, we have observed two practices: first, we have kept the observation term $A:=\frac{1}{2} \int_{\Omega_{d}}(T-$ 
$\left.T_{d}\right)^{2} d x$ dominant in (2.4); second, we have kept the regularization term $\beta_{1} B:=\frac{\beta_{1}}{2} \int_{\Gamma_{i}}|\mathbf{g}|^{2} d s$ comparable to $\beta_{2} C:=\frac{\beta_{2}}{2} \int_{\Gamma_{i}}|\nabla \mathbf{g}|^{2} d s$. These two goals can be achieved simultaneously by taking

$$
\left\{\begin{array}{l}
\beta_{1}=O\left(\frac{1}{300} \cdot \frac{A}{B}\right) \\
\beta_{2}=O\left(\beta_{1} \cdot \frac{B}{C}\right) .
\end{array}\right.
$$

In this test case with the partial observation operator and before the first optimization iteration, $A=1.97 \times 10^{-1}, B=1.58 \times 10^{-2}$, and $C=2.81 \times 10^{-1}$. Following (5.2), we have chosen $\beta_{1} \cong O\left(4.15 \times 10^{-2}\right)$ and $\beta_{2} \cong O\left(2.33 \times 10^{-3}\right)$.

In Figure 7.12, we study the sensitivity of the computed control with respect to the regularization parameters $\beta_{1}$ and $\beta_{2}$. Starting from the smallest possible regularization parameters $\beta_{1}=8 \times 10^{-3}$ and $\beta_{2}=8 \times 10^{-4}$ that allow the L-BFGS method to converge, the larger the $\beta_{1}$ and $\beta_{2}$ are, the less dominant the observation term in (2.4) becomes over the regularization term and thus the further the computed controls are from the exact control. It follows the conclusion that regularization has an effect on the convergence of numerical optimization algorithms. In comparison to the L-BFGS method, the smallest possible regularization parameters that allow the smoothed Steepest Descent method to converge are $\beta_{1}=4 \times 10^{-2}$ and $\beta_{2}=4 \times 10^{-3}$. From Table 7.4, we see that when $\beta_{1}$ and $\beta_{2}$ increase, the regularity of the optimization problem increases and thus the counts of nonlinear optimization iterations and line search iterations decrease for both the smoothed Steepest Descent method and the L-BFGS method. And the regularization has a bigger impact on the smoothed Steepest Descent method than that on the L-BFGS method.

\subsection{Numerical Test Problem 2}

Solving this second suite of test problems is a much more challenging task for our optimization algorithms. In the channel flow case, the target temperature $T_{d}$ is generated by the piecewise quadratic inflow boundary control profile $\mathbf{g}=\left(g_{x}, 0\right)^{T}$, where

$$
g_{x}= \begin{cases}-32\left(y-\frac{9}{16}\right)^{2}+\frac{9}{8} & y \in\left[\frac{3}{8}, \frac{3}{4}\right], \\ -32\left(y-\frac{3}{16}\right)^{2}+\frac{9}{8} & y \in\left[0, \frac{3}{8}\right] .\end{cases}
$$



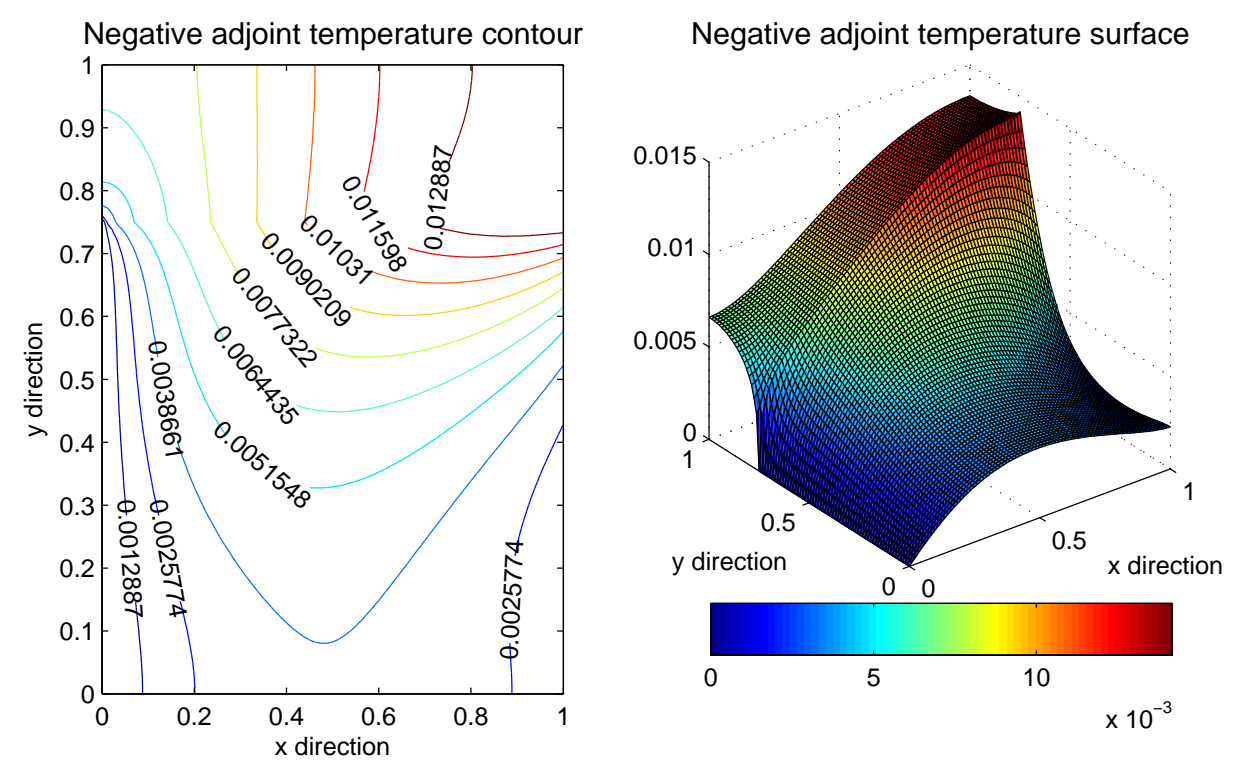

Figure 7.9: Last adjoint temperature solution of the L-BFGS method with $\Omega_{d}=[0.075,1] \times\{0.75\}$.

Adjoint velocity vector $\mathrm{x}$ component

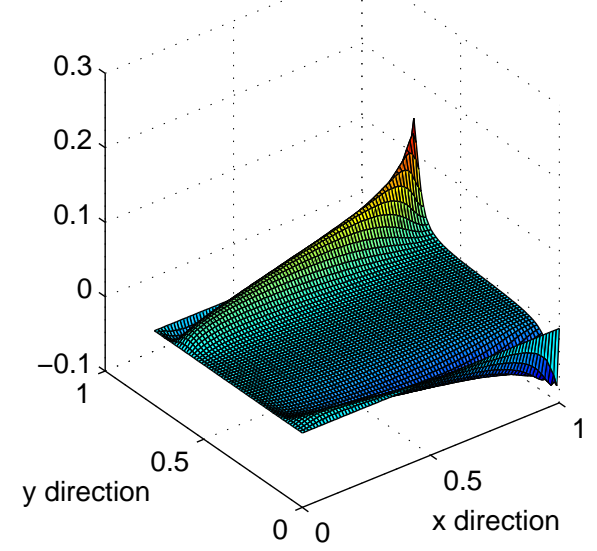

Adjoint velocity vector y component

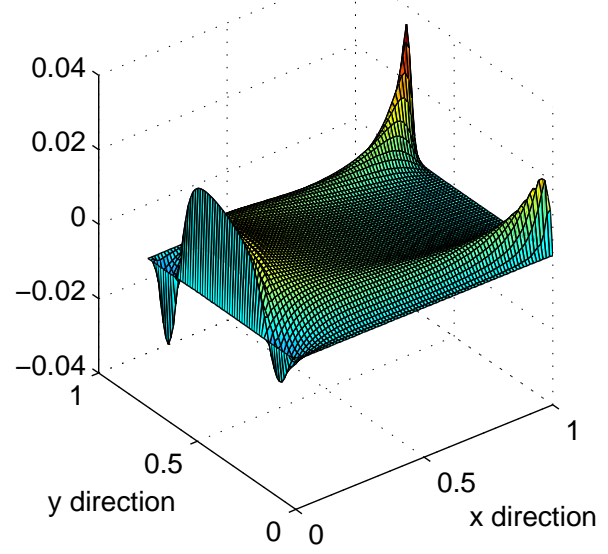

Figure 7.10: Last adjoint velocity solution of the L-BFGS method with $\Omega_{d}=[0.075,1] \times\{0.75\}$. 


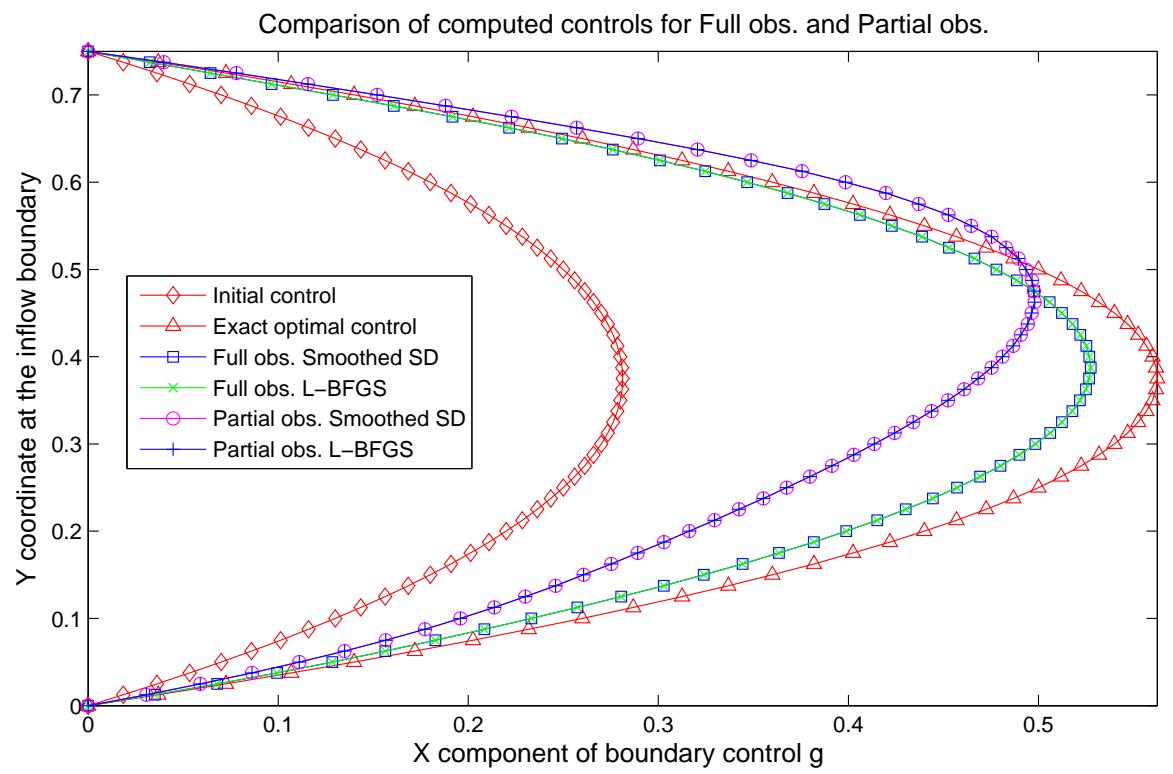

Figure 7.11: Computed controls for both the smoothed Steepest Descent (SD) method and the LBFGS method. In the full observation (obs.) case, $\Omega_{d}=\Omega_{2}$ and $\beta_{1}=\beta_{2}=1$. In the partial observation case, $\Omega_{d}=[0.075,1] \times\{0.75\}, \beta_{1}=5 \times 10^{-2}$ and $\beta_{2}=5 \times 10^{-3}$. The exact optimal control profile is described in (7.1).

\begin{tabular}{cccccc}
\hline \multicolumn{2}{c}{ Regularization } & \multicolumn{2}{c}{ Smoothed SD } & \multicolumn{2}{c}{ L-BFGS } \\
\hline$\beta_{1}$ & $\beta_{2}$ & Opt. & L.s. & Opt. & L.s. \\
\hline $8 \times 10^{-3}$ & $8 \times 10^{-4}$ & - & - & 15 & 10 \\
$4 \times 10^{-2}$ & $4 \times 10^{-3}$ & 54 & 165 & 9 & 4 \\
$5 \times 10^{-2}$ & $5 \times 10^{-3}$ & 36 & 101 & 8 & 4 \\
\hline
\end{tabular}

Table 7.4: Effects of regularization on optimization (Opt.) and line search (L.s.) iterations counts for the smoothed Steepest Descent method and the L-BFGS method with the partial observation domain $\Omega_{d}=[0.075,1] \times\{0.75\}$. 


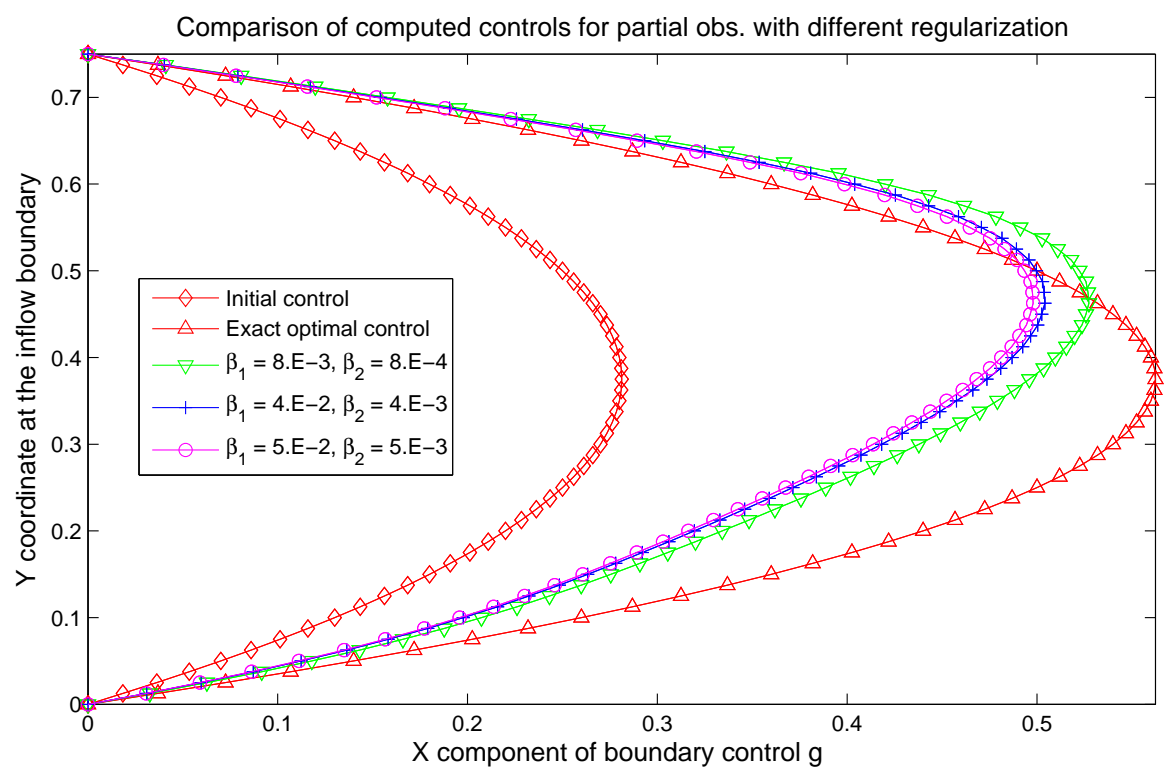

Figure 7.12: Computed controls for the L-BFGS method with the partial observation domain $\Omega_{d}=$ $[0.075,1] \times\{0.75\}$. The exact optimal control profile is described in (7.1).

See Figure 7.19 for the plot of $g_{x}$. The initial control in (2.1) is set to be the Poiseuille flow profile $\left(1.5 y-2 y^{2}, 0\right)^{T}$ on $\Gamma_{i}=\{0\} \times[0,0.75]$. Our aim is to match the fluid temperature with the target temperature $T_{d}$ and approximate the optimal control in (7.3) via minimizing the objective function $\mathcal{J}(\mathbf{g})$. As shown in Figure 7.19, the initial control is significantly different from the exact optimal control in shape. Therefore, we consider this test as a difficult problem for the optimization scheme. In Section 7.3.1, we show that with the regularization continuation technique discussed in Section 6.5, the L-BFGS method can solve this demanding problem to a high accuracy.

We set up the test model with the following data: $R e=1000, k_{s}=1, k_{f}=0.1$ and the heating source $q=6$ in the solid domain. The computational domain consists of $\Omega_{2}=[0,1] \times[0,0.75]$ and $\Omega_{1}=[0,1] \times[0.75,1]$, and the observation domain $\Omega_{d}$ is set to be $\Omega_{2}$ in (2.4). The mesh layout is the same as that of the test problem 1 . 


\subsubsection{Numerical Continuation on the regularization parameters}

In this test case, from (7.2), we have $A=2.15 \times 10^{1}, B=1.58 \times 10^{-2}$, and $C=2.81 \times 10^{-1}$, which lead to $\beta_{1} \cong O(4.53)$ and $\beta_{2} \cong O\left(2.54 \times 10^{-1}\right)$. It turns out that the smallest regularization parameters that make the L-BFGS method to converge are $\beta_{1}=0.1$ and $\beta_{2}=0.1$. It is evident that choosing the right amount of regularization is important for this boundary control problem. In fact, choosing penalty parameters as small as $\beta_{1}=0.1$ and $\beta_{2}=0.01$ provides too little regularization, producing a nonsmooth control profile and causing the line search iteration of the L-BFGS method to fail to identify a feasible stepsize. However, for a slightly larger amount of regularization, namely, $\beta_{1}=0.1$ and $\beta_{2}=0.1$, the L-BFGS method converges only to a suboptimal control profile without any resemblance to the shape of the two adjacent Poiseuille-flow peaks in the optimal control. This is due to an excess of regularization. See the Phase-1 control plot in Figure 7.13.

Our solution strategy is to start with a sufficient amount of regularization that allows the L-BFGS method to produce a converged suboptimal control profile. Then, we use regularization continuation strategy described in Algorithm 6.5.1. We emphasize that we do not force the optimization scheme to locate the global minimum at the current continuation phase; instead, we are satisfied with reducing both the objective function and the reduced gradient to a certain extent as long as an improved control profile can be produced. The difficulty of solving the original problem is alleviated by solving multiple easier subproblems. During each continuation phase, we fix the amount of regularization and use the suboptimal control from the previous phase as an initial guess to solve the subproblem at current phase, but only up to a relaxed tolerance. Because of the progressive reduction in the amount of regularization at the end of each continuation phase, the observation function $\frac{1}{2} \int_{\Omega_{d}}\left(T-T_{d}\right)^{2} d \mathbf{x}$ remains relatively dominant, but not so dominant that there is not enough regularization and that the optimization iteration would fail. This sustained dominance forces the optimization scheme to consistently reduce the observation function value (and thus the objective function value), towards locating an accurate approximation of the optimal boundary control profile.

In this numerical study, we have implemented the L-BFGS method with a 5-phase regularization continuation strategy, namely, $k=5$ in Algorithm 6.5.1. Starting with $\beta_{1}=\beta_{2}=0.1$ at the zeroth phase, the reduction factor is $c=10^{-1}$ for both parameters. The initial control profile $\mathbf{g}_{k+1}^{0}$ at the 
phase $k+1$ is the computed optimal control $\mathbf{g}_{k}^{\star}$ at phase $k$. From Figure 7.13, we can see that as the continuation phases evolve, the computed control profiles progressively approach to the exact optimal control profile that is shown in the Figure 7.19. For a better illustration, the $x$ components of the velocity field induced by the boundary control at the end of each phase are shown in Figure 7.14. Finally, Figure 7.15 dispays the progressive reduction in the objective function along the continuation path.

We have also carried out experiments on the sensitivities of the optimization scheme with respect to different starting regularization parameters $\left(\beta_{1}, \beta_{2}\right)$ and different reduction factors $c$ in Algorithm 6.5.1. It turns out that the quality of computed control profiles degrades and the counts of optimization iterations and line search iterations go down when the starting regularization parameters increase, with $r t o l=10^{-2}$ and the reduction factor $c=0.1$ fixed, as shown in Figure 7.16 and Table 7.5. The relationship here between the regularization parameters and the iterations counts are similar to that in Section 7.2.4. The computed control profiles generated by different reduction factors $c$ are displayed in Figure 7.17, which shows that the reduction factor $c$ has a direct impact on the quality of the computed controls when $r t o l=10^{-2}$ and the starting regularization parameters $\beta_{1}=0.1$ and $\beta_{2}=0.1$. The quality of the computed controls degrades as $c$ increases, and this indicates the excess of regularization when the reduction factor $c$ is not small enough. We list the iterations counts of the L-BFGS method with 5-phase regularization continuation for different reduction factors $c$ in Table 7.6.

We have studied the sensitivity of the optimization scheme with respect to different levels of Gaussian white noise added to the target temperature. In real applications, measurements are subject to uncertainty. Adding random perturbations to the target temperature is a means of demonstrating robustness with respect to such uncertainty. We thus avoid committing an "inverse crime" of the type where the target profile is synthesized by the same model that establishes the control profile [37]. We assume that the target temperature is generated by the abstract operator $\mathbf{F}(\mathbf{g})$ with additive Gaussian white noise

$$
T_{d}=\mathbf{F}(\mathbf{g})+e,
$$

where $e$ is a random vector, with independent and identically distributed normal components of 
mean 0 and standard deviation $S D \cdot \max (|\mathbf{F}(\mathbf{g})|)$. We have generated the computed controls for various noise levels $S D=0.005,0.01,0.02,0.04$, and 0.08 , respectively. We display the computed control profiles in Figure 7.18. For the lower noise levels $S D=0.005,0.01$, and 0.02, the computed controls are of good quality. As the noise level is increased, the computed control starts to degrade, since noise are more pronounced when $S D$ is large.

We conclude this section with the importance of avoiding oversolving the subproblems when the L-BFGS method is combined with regularization continuation. It is evident from Figure 7.19 that for the boundary control of CHT processes, rtol, the relaxed relative tolerance on the reduced gradient, as large as $10^{-2}$ is sufficient. Any further tightening on this tolerance does not improve the computed control profile, but purely taxes the computational cost. This further reflects the idea of the regularization continuation: only solve the current subproblem approximately to obtain a better initial control profile for the next subproblem. Table 7.7 provides detailed information on the computational expense. It is evident that choosing $r t o l=10^{-2}$ can significantly avoid oversolving. We note that relaxing the $r t o l$ is equivalent to introducing inexactness in the optimization loop. In fact, inexactness of the state and adjoint solvers can also be explored to further enhance the efficiency of the optimization scheme. See Remark 5.2.1 for references.

\subsubsection{Boundary Control of CHT flow past a square cylinder}

To demonstrate the effectiveness and robustness of our optimization schemes on a different geometry and on a more complex flow velocity field, we set up the boundary control model for a cold confined flow past a hot square cylinder. For further discussion on the flow past a square cylinder, see [32]. The test model has the following data: $v=0.01, k_{s}=3$ and $k_{f}=0.4$. The physical domain in Figure 7.20 consists of $\Omega_{2}=[0,8] \times[-1,1]$ and $\Omega_{1}=[1.25,1.75] \times[-0.25,0.25]$. It has a length $L=8$ and height $H=2$. A solid square with a diameter $D=0.5$ is placed at $(1.5,0)$ with its center on this point. This corresponds to a blockage ratio $R:=D / H=1 / 4$ and Reynolds number $R e_{D}:=D \bar{U} / v=50$, where $\bar{U}$ is the magnitude of the mean velocity. We take the heating source in the solid domain $\Omega_{1}$ as a constant value $q=16$. To the walls of the solid square, $\Gamma_{k}, k=1,2,3,4$, and the wall of the channel, $\Gamma_{5}$ and $\Gamma_{6}$, we have applied no-slip boundary conditions for the fluid 


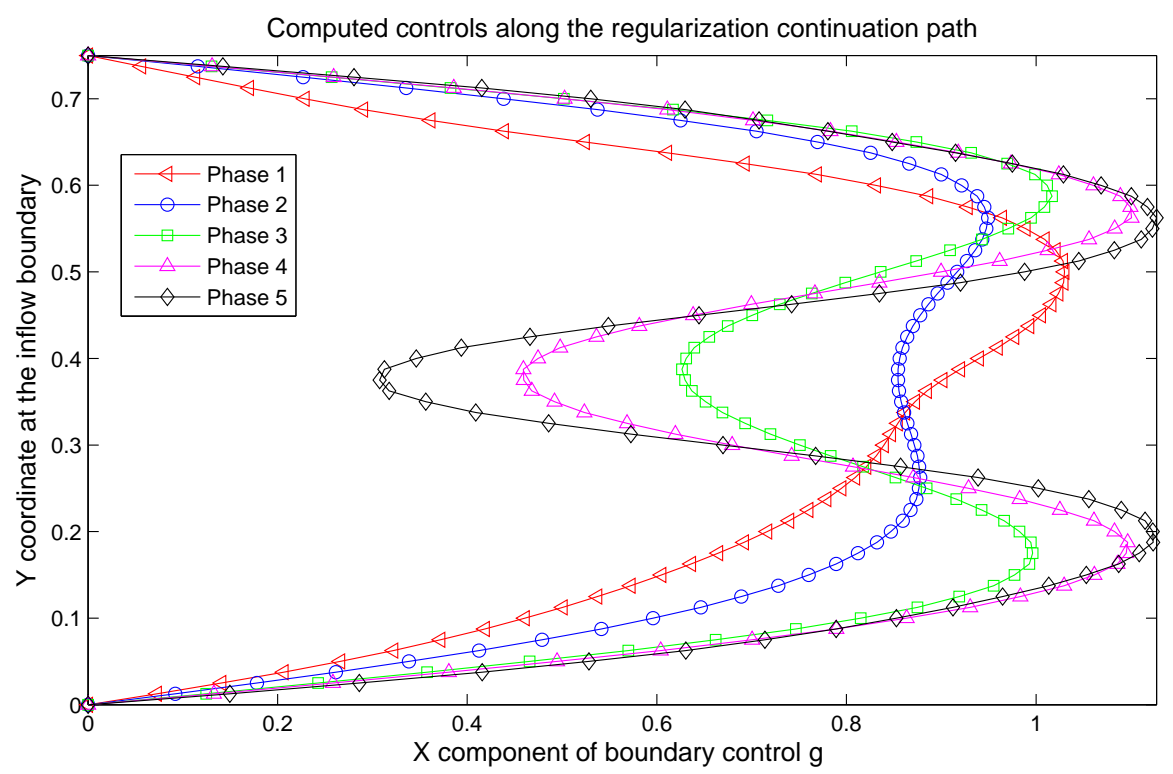

Figure 7.13: Computed controls of Numerical Test 2 using the L-BFGS method with 5-phase regularization continuation. We have set $r t o l=10^{-2}$ for the L-BFGS method to generate this plot.

\begin{tabular}{ccccccccccc}
\hline \multicolumn{1}{c}{$\beta_{i}=1 \times 10^{-1}$} & \multicolumn{2}{c}{$\beta_{i}=2 \times 10^{-1}$} & \multicolumn{2}{c}{$\beta_{i}=4 \times 10^{-1}$} & \multicolumn{2}{c}{$\beta_{i}=8 \times 10^{-1}$} & \multicolumn{2}{c}{$\beta_{i}=1.6$} \\
\hline Phase & Opt. & L.s. & Opt. & L.s. & Opt. & L.s. & Opt. & L.s. & Opt. & L.s. \\
\hline 1 & 16 & 11 & 8 & 6 & 8 & 4 & 6 & 4 & 4 & 4 \\
2 & 8 & 12 & 5 & 9 & 5 & 7 & 5 & 6 & 4 & 5 \\
3 & 9 & 20 & 8 & 18 & 6 & 16 & 7 & 13 & 6 & 10 \\
4 & 11 & 36 & 10 & 31 & 9 & 27 & 9 & 23 & 8 & 20 \\
5 & 18 & 59 & 16 & 53 & 13 & 45 & 12 & 39 & 11 & 32 \\
\hline Total & 62 & 138 & 44 & 117 & 41 & 99 & 39 & 85 & 33 & 71 \\
\hline
\end{tabular}

Table 7.5: Optimization (Opt.) and line search (L.s.) iterations counts in Numerical Test 2 using the L-BFGS method with 5-phase regularization continuation. The comparison is made for different starting regularization parameters $\left(\beta_{1}, \beta_{2}\right)$. The index $i$ runs over 1 and 2 . 
Phase 1: velocity $x$ component

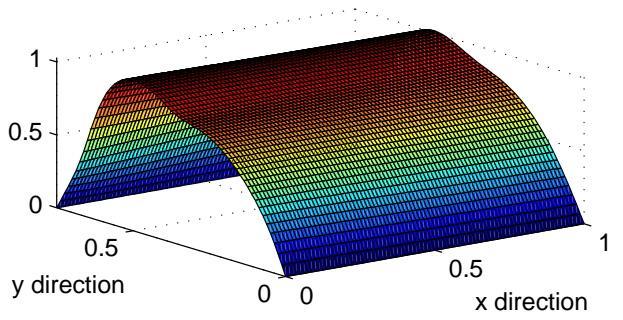

Phase 3: velocity $x$ component

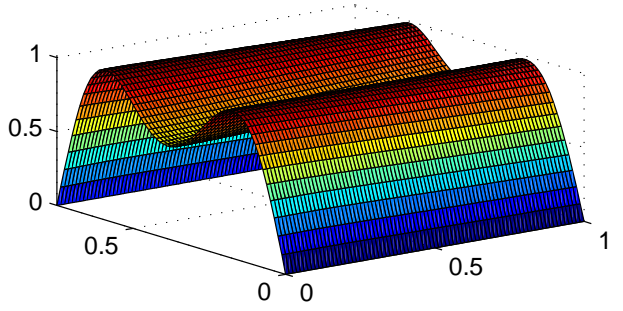

Phase 5: velocity $x$ component

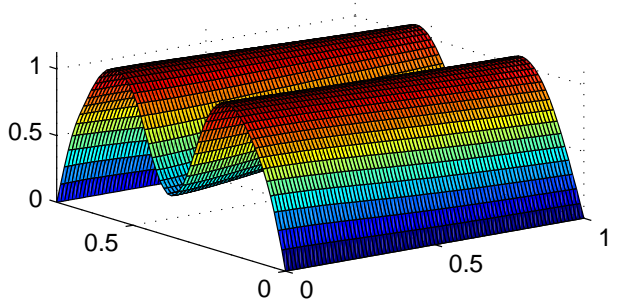

Phase 2: velocity $\mathrm{x}$ component

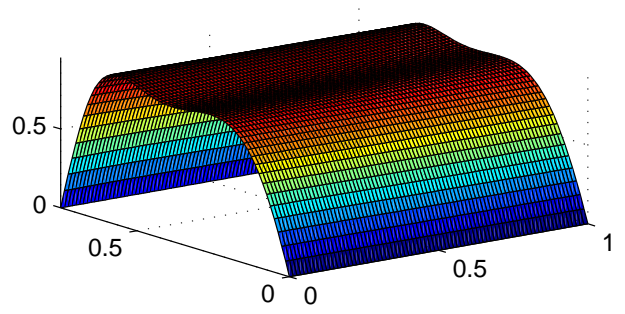

Phase 4: velocity $x$ component

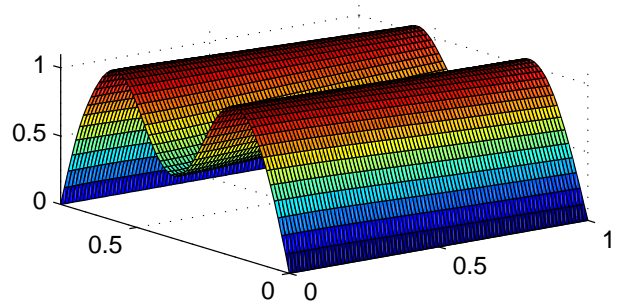

Figure 7.14: Computed $x$ components of the fluid velocity in Numerical Test 2 using the L-BFGS method with 5-phase regularization continuation. We have set $r t o l=10^{-2}$ for the L-BFGS method to generate this plot. 


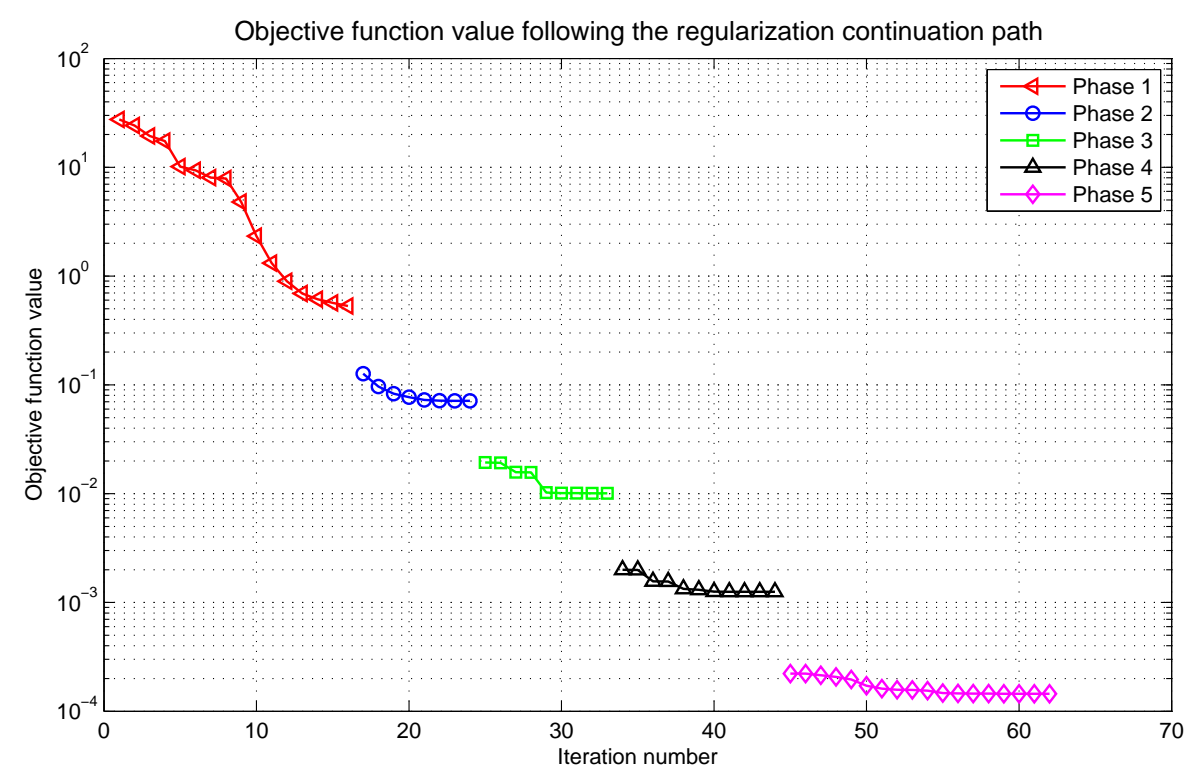

Figure 7.15: History of the objective function value in Numerical Test 2 using the L-BFGS method with 5-phase regularization continuation. We have set $r t o l=10^{-2}$ for the L-BFGS method to generate this plot.

\begin{tabular}{ccccccccc}
\hline \multicolumn{1}{c}{$c=1 \times 10^{-1}$} & $c=2 \times 10^{-1}$ & $c=4 \times 10^{-1}$ & $c=8 \times 10^{-1}$ \\
\hline & Opt. & L.s. & Opt. & L.s. & Opt. & L.s. & Opt. & L.s. \\
\hline Total & 62 & 138 & 45 & 89 & 36 & 59 & 34 & 36 \\
\hline
\end{tabular}

Table 7.6: Optimization (Opt.) and line search (L.s.) iterations counts in Numerical Test 2 using the L-BFGS method with 5-phase regularization continuation. The comparison is made for different reduction factors $c$. The quality of the computed controls degrades as $c$ decreases, as shown in Figure 7.17. 


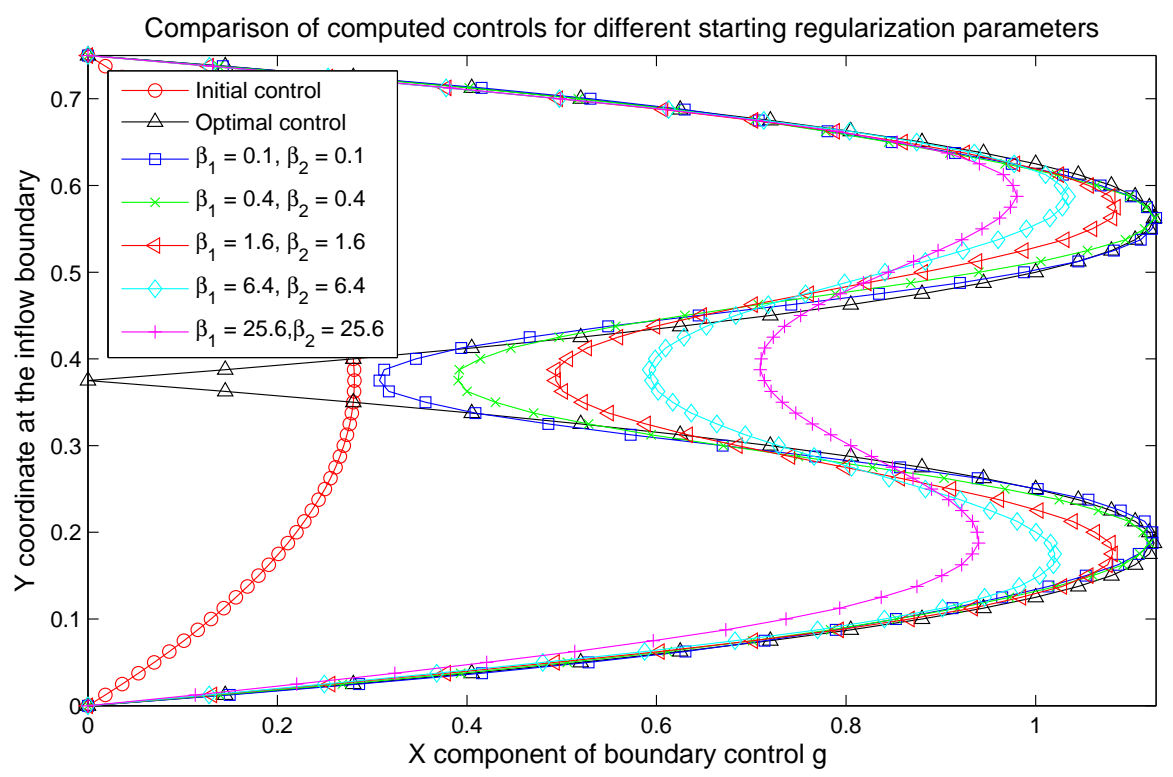

Figure 7.16: Computed controls of Numerical Test 2 using the L-BFGS method with 5-phase regularization continuation. The comparison is made for different starting regularization parameters $\left(\beta_{1}, \beta_{2}\right)$. The exact optimal control profile is described in (7.3).

\begin{tabular}{ccccccccc}
\hline & Regularization & \multicolumn{2}{c}{$r t o l=10^{-2}$} & \multicolumn{2}{c}{$r t o l=10^{-3}$} & \multicolumn{2}{c}{$r t o l=10^{-4}$} \\
\hline Phase & $\beta_{1}$ & $\beta_{2}$ & Opt. & L.s. & Opt. & L.s. & Opt. & L.s. \\
\hline 1 & $10^{-1}$ & $10^{-1}$ & 16 & 11 & 19 & 11 & 20 & 11 \\
2 & $10^{-2}$ & $10^{-2}$ & 8 & 12 & 9 & 12 & 9 & 12 \\
3 & $10^{-3}$ & $10^{-3}$ & 9 & 20 & 10 & 20 & 12 & 20 \\
4 & $10^{-4}$ & $10^{-4}$ & 11 & 36 & 14 & 34 & 15 & 35 \\
5 & $10^{-5}$ & $10^{-5}$ & 18 & 59 & 25 & 93 & 33 & 161 \\
\hline Total & - & - & 62 & 138 & 77 & 170 & 89 & 239 \\
\hline
\end{tabular}

Table 7.7: Optimization (Opt.) and line search (L.s.) iterations counts in Numerical Test 2 using the L-BFGS method with 5-phase regularization continuation. The comparison is made for different relative tolerances in the L-BFGS method. 


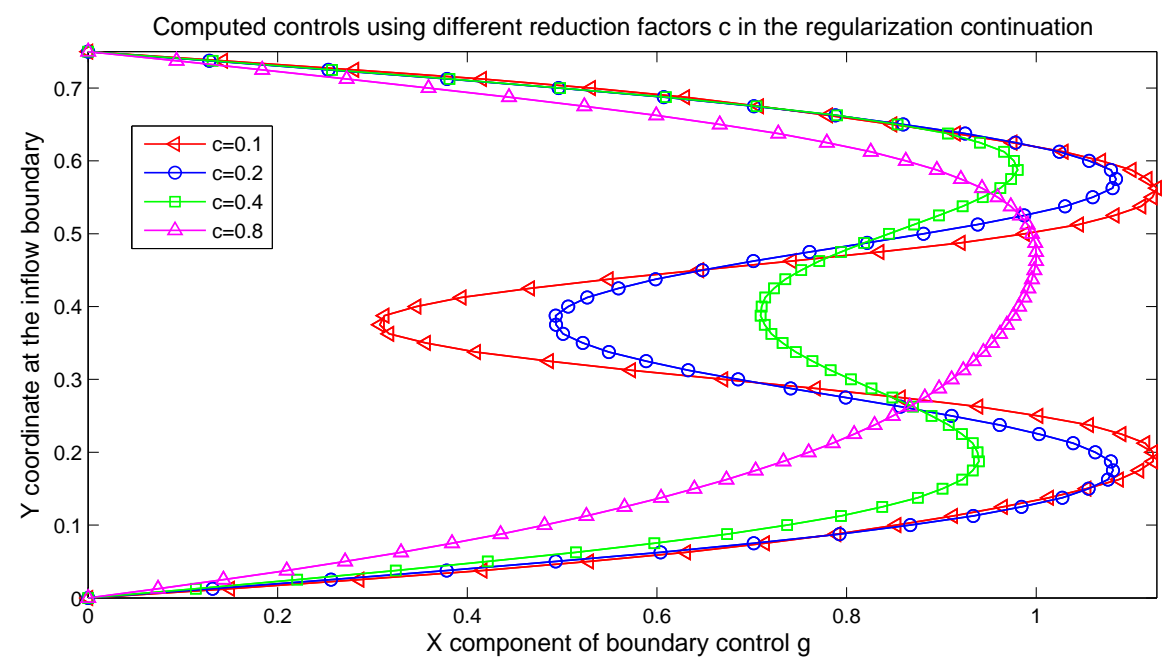

Figure 7.17: Computed controls of Numerical Test 2 using the L-BFGS method with 5-phase regularization continuation. The comparison is made for different reduction factors $c$ in Algorithm 6.5.1. The exact optimal control profile is described in (7.3).

velocity $\mathbf{u}$. At the outlet of the channel $\Gamma_{o}$, we have posed the stress-free boundary condition for $\mathbf{u}$. The initial control $\mathbf{g}$ in (2.1) is set to be the parabolic flow profile $\left(1-y^{2}, 0\right)^{T}$ on $\Gamma_{i}=\{0\} \times[-1,-1]$. The exact optimal control is given by

$$
\mathbf{g}=4\left(1-y^{2}, 0\right)^{T}
$$

For the temperature system in the fluid-solid region, $\Gamma_{5}, \Gamma_{6}$, and $\Gamma_{o}$ are adiabatic boundaries. The fluid-solid thermal interface consists of $\Gamma_{k}, k=1,2,3,4$, and its boundary conditions are the continuity of temperature and heat flux. See similar boundary conditions in (2.2)-(2.3). On the inflow boundary $\Gamma_{i}$, we prescribe the fluid temperature. The target temperature $T_{d}$ in (2.4) is calculated using the fluid velocity induced by the optimal control profile in (7.5) and is shown in Figure 7.21 .

We set the observation domain as $\Omega_{d}=\Omega_{2} \cap\left(\Omega_{1}\right)^{c}$. The IFISS package $[47,117]$ has been used for the finite element assembly. In the mesh generation, the fluid-solid domain is discretized with 128 and $32 \mathrm{Q} 2$ elements in $x$ and $y$ directions respectively. In a single Q2 cell, there are 9 degrees of freedom (DOF) for each component of the triple $\left(u_{x}, u_{y}, T\right)$. The fluid pressure is discretized with 


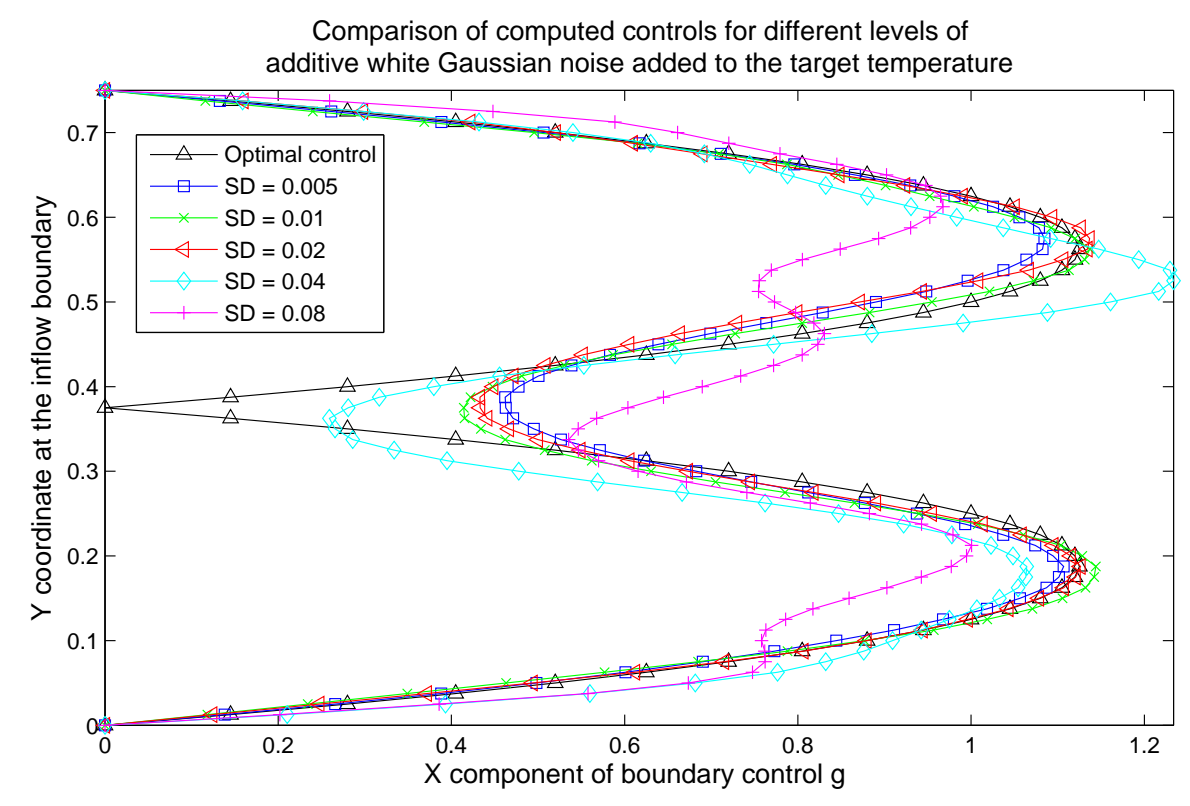

Figure 7.18: Computed controls of Numerical Test 2 using the L-BFGS method with regularization continuation. The comparison is made for different levels of white Gaussian noise added to the target temperature. The exact optimal control profile is described in (7.3). 


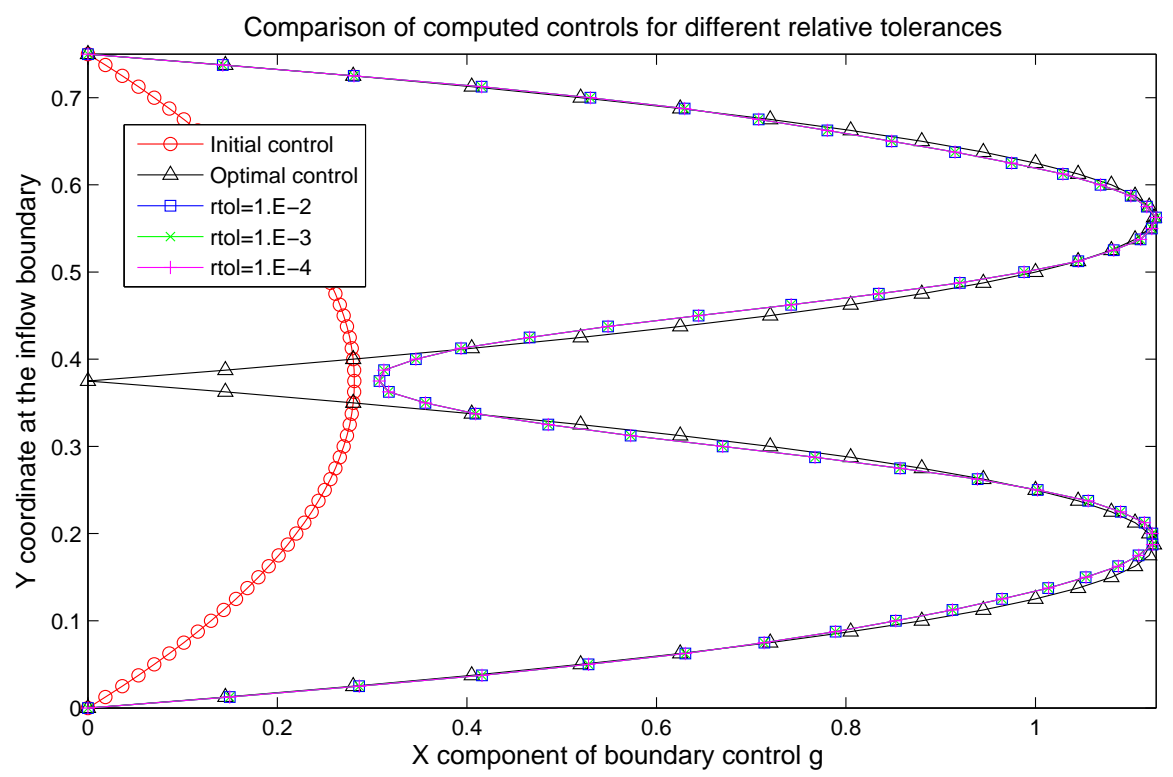

Figure 7.19: Computed controls of Numerical Test 2 using the L-BFGS method with 5-phase regularization continuation. The comparison is made for different relative tolerances in the L-BFGS method. The exact optimal control profile is described in (7.3). 
128 Q1 elements in $x$ and 32 in $y$ direction on the fluid domain $\Omega_{2}$. Each Q1 cell has 4 DOF.

To assemble the objective function (2.4), we use the strategy in (7.2). Before the first optimization iteration, the terms in the objective function (2.4) and equation (7.2) are $A=4.70 \times 10^{2}$, $B=5.33 \times 10^{-1}$, and $C=1.33$. Therefore, we have chosen the regularization parameters: $\beta_{1}=3$ and $\beta_{2}=1.2$. Also, it is evident that the initial objective function value is quite large and the initial control is far from optimal, when the optimal control (7.5) and the initial control $\left(1-y^{2}, 0\right)^{T}$ are in use for the test model. In the numerical experiments, regularization parameters that are in the vicinity of $\left(\beta_{1}=3, \beta_{2}=1.2\right)$ and thus sufficiently large to make the optimization scheme converge are only able to provide suboptimal temperature profiles. In Figure 7.23, we display the computed temperature profile generated by the L-BFGS method without regularization continuation. The relative tolerance is set to be $r t o l=10^{-6}$, which is much smaller than the relaxed tolerance $10^{-2}$ in the $k$-phase L-BFGS method. However, merely reducing the relative tolerance does not provide any adjustment to keep the dominance of the temperature mismatch over the regularization terms in the equation (2.4). Therefore, to obtain better computed temperature profiles, we apply the L-BFGS method with 2-phase regularization continuation and with $r t o l=10^{-2}$ and $c=10^{-1}$ to this test model. It takes 8 optimization iterations and 12 line search reductions in total to recover the target temperature profile. We display the target temperature, initial temperature, and computed optimal temperature profiles in Figure 7.21, 7.22, and 7.24. It is evident from Figures 7.21-7.24 that the computed temperature profile generated by the 2-phase L-BFGS method has a higher quality than the one generated by the L-BFGS method without regularization continuation. The computed control profiles after the first and the second phase of the L-BFGS method with 2-phase regularization continuation are shown in Figure 7.25, from which it is evident that the optimal control profile has been identified successfully by the optimization scheme. 


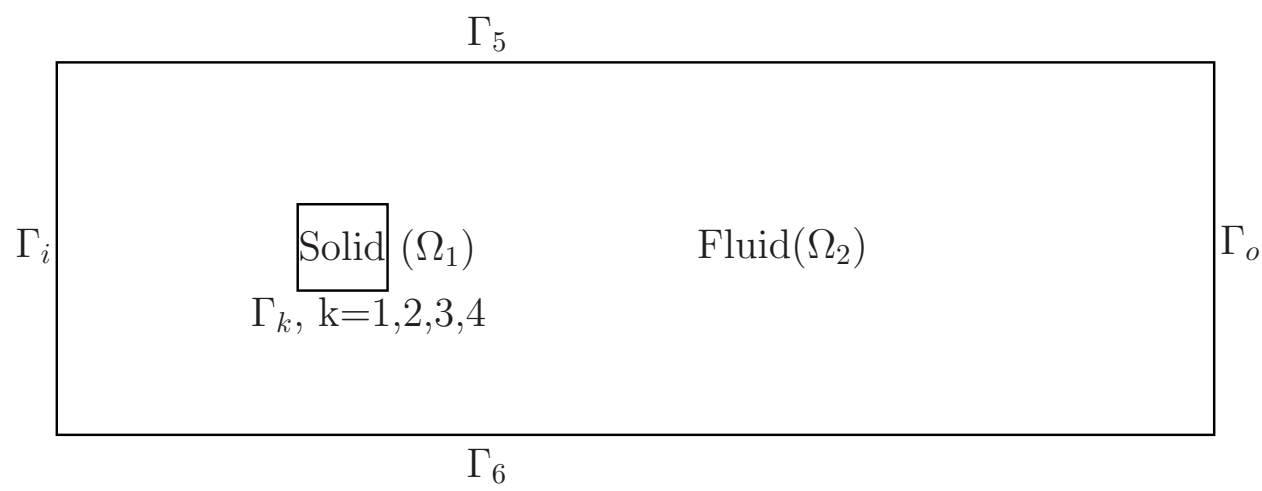

Figure 7.20: Physical domain of the flow past a square cylinder.
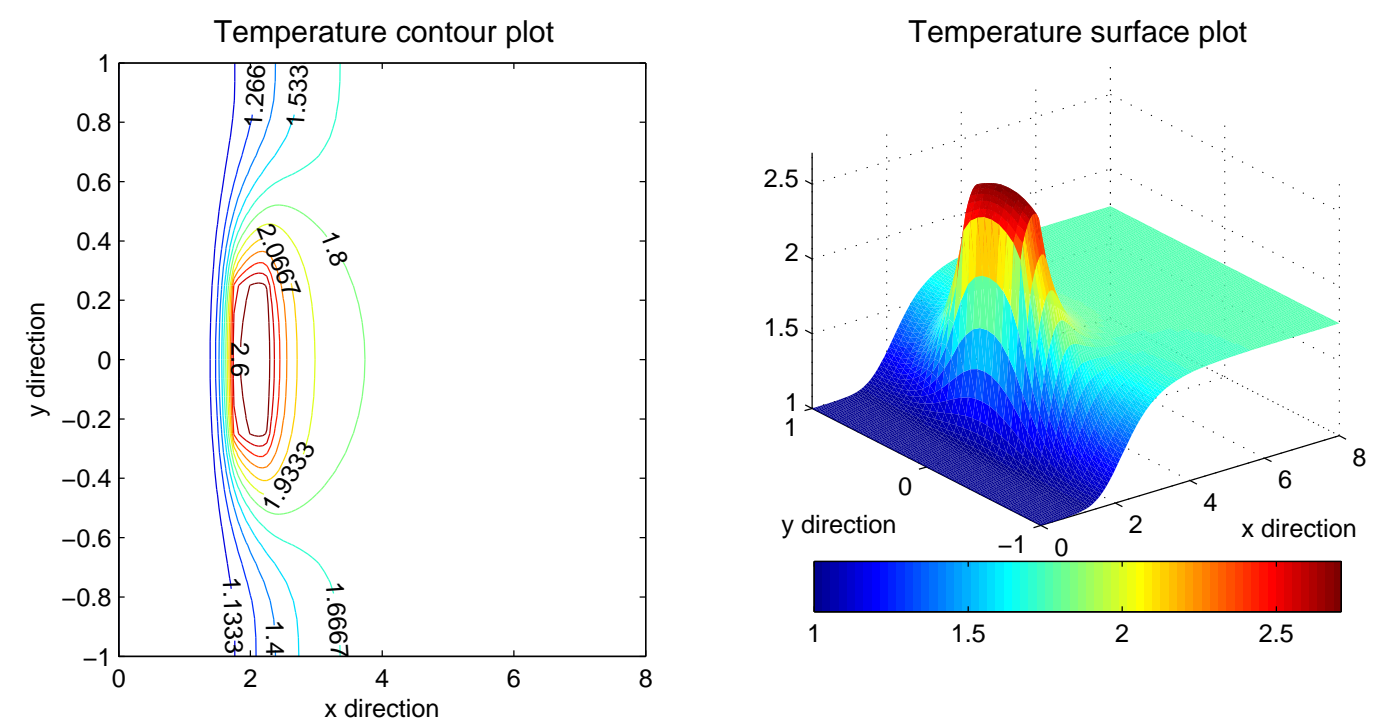

Figure 7.21: Target temperature in the boundary control of CHT flow past a square cylinder. 

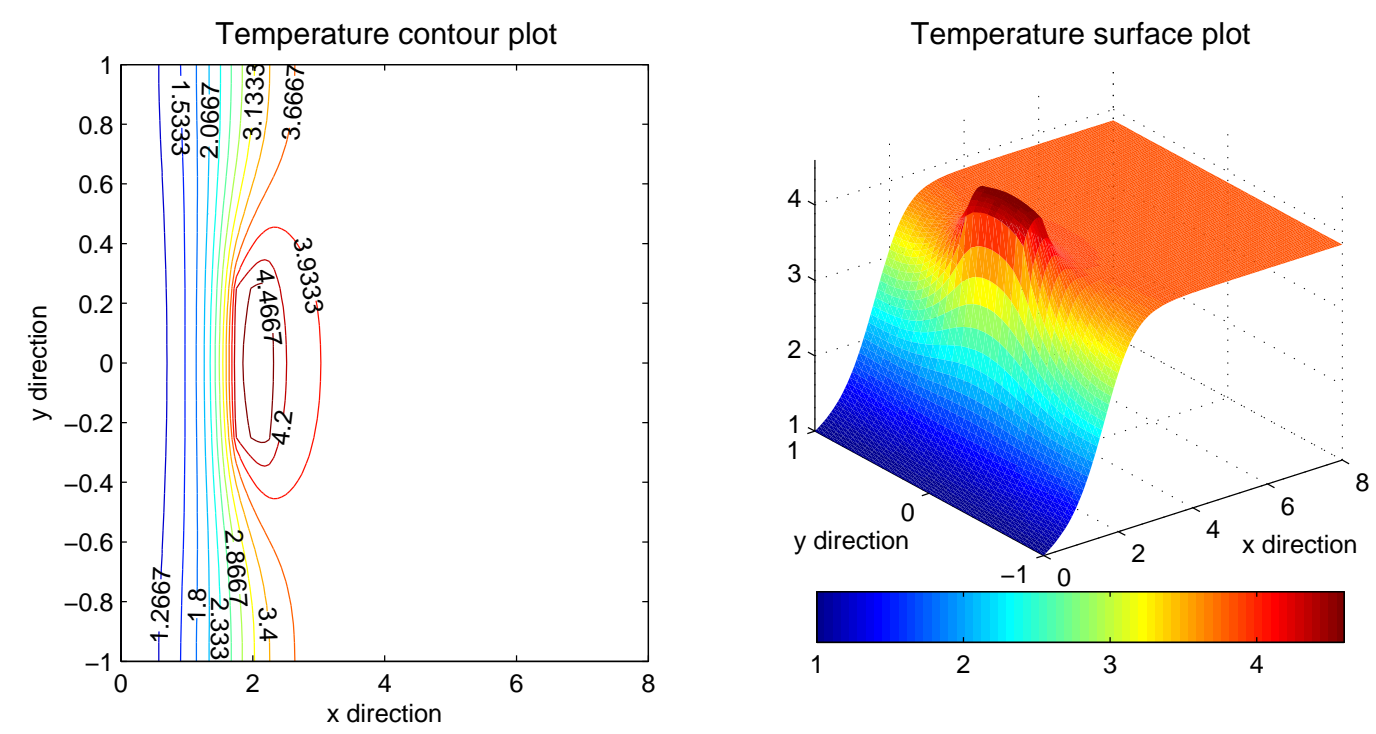

Figure 7.22: Initial temperature in the boundary control of CHT flow past a square cylinder. 

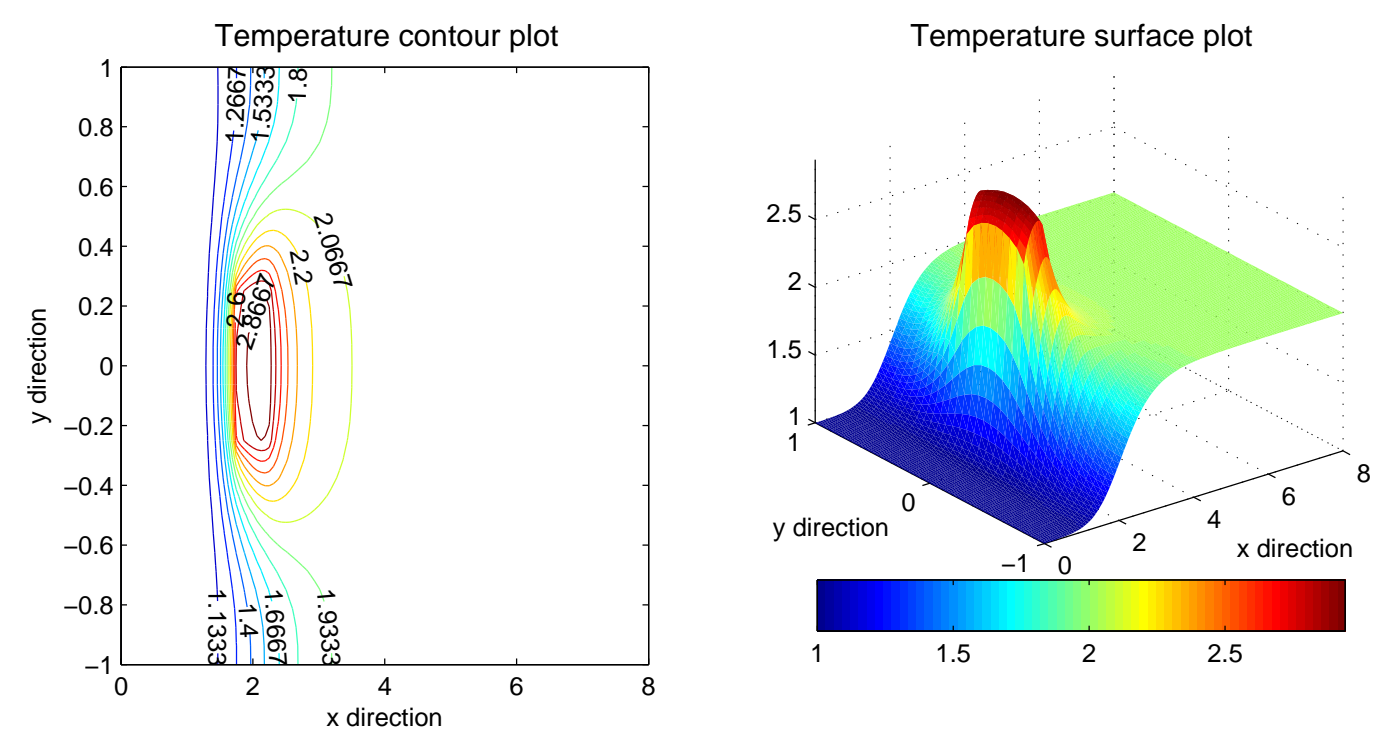

Figure 7.23: Temperature induced by the computed optimal control in the boundary control of CHT flow past a square cylinder, using the L-BFGS method without regularization continuation. The relative tolerance is $r t o l=10^{-6}$. 

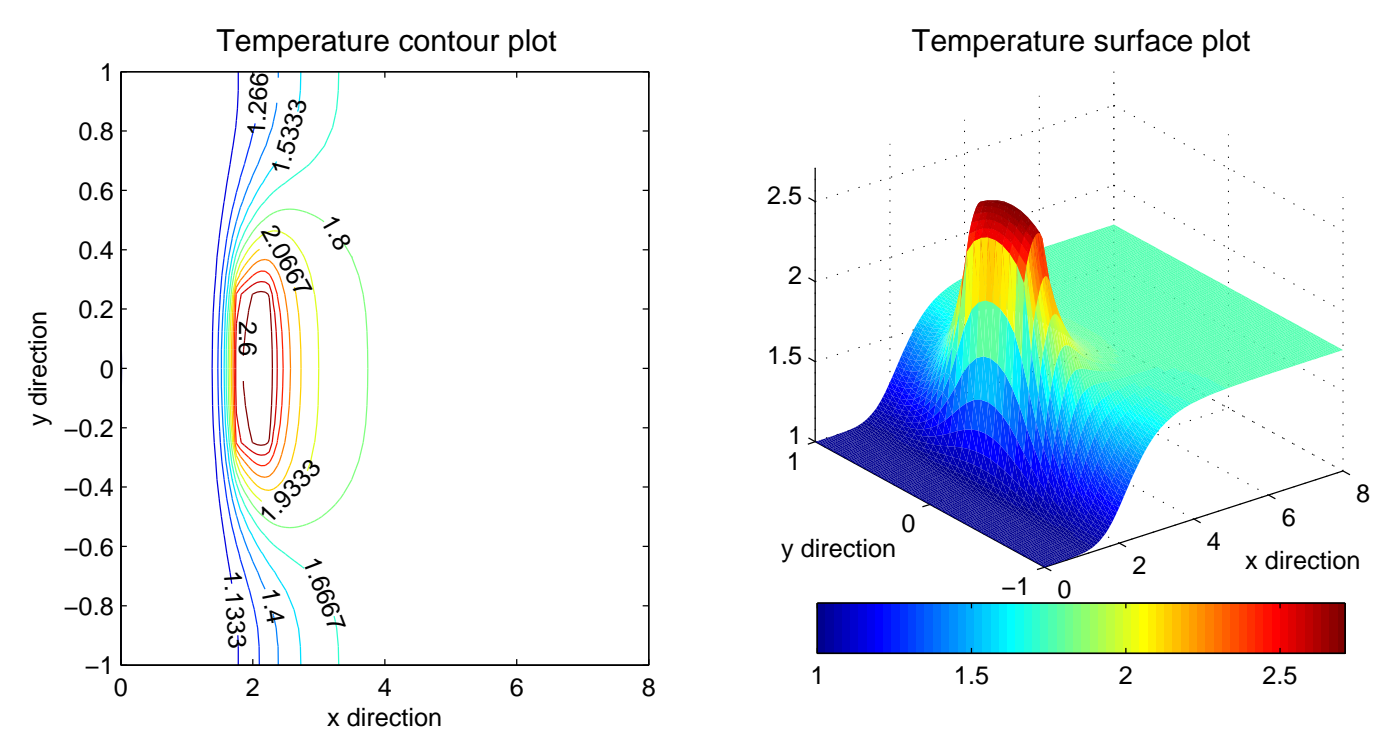

Figure 7.24: Temperature induced by the computed optimal control in the boundary control of CHT flow past a square cylinder, using the 2-phase L-BFGS method. The computed temperature profile in this case matches the target temperature better than the profile generated by the L-BFGS method without regularization continuation in Figure 7.23. 


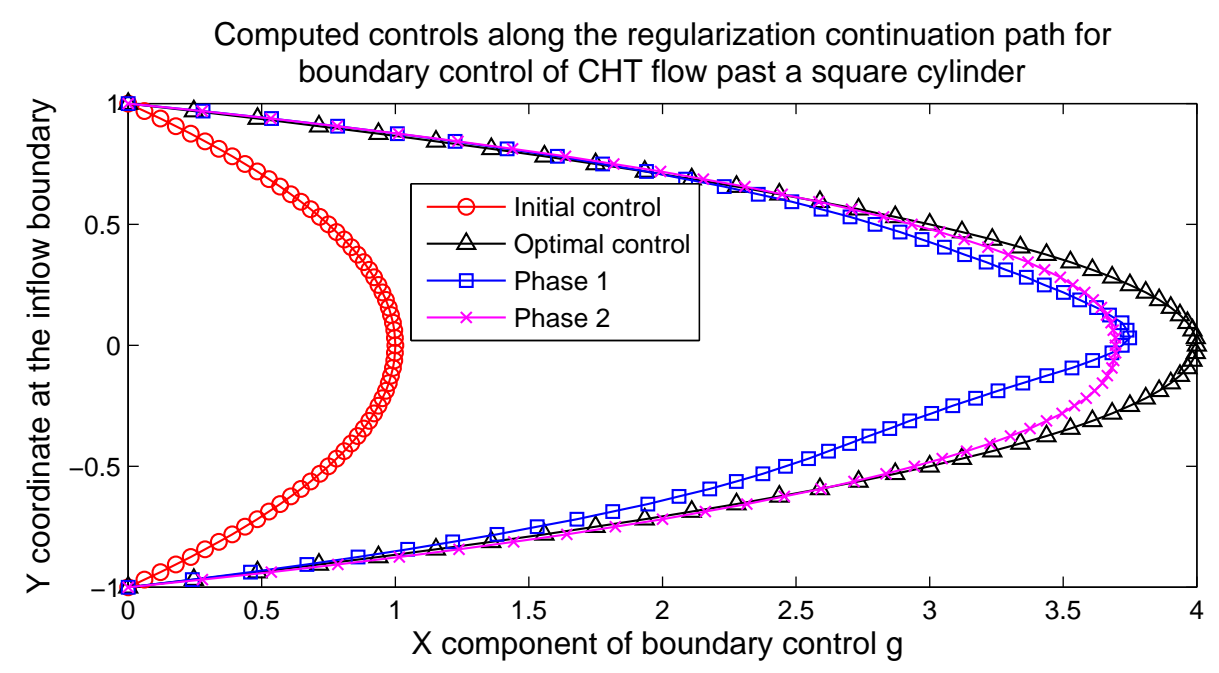

Figure 7.25: Computed controls of CHT flow past a square cylinder using the L-BFGS method with 2-phase regularization continuation. We have set $r t o l=10^{-2}$ for the L-BFGS method to generate this plot. The exact optimal control profile is described in (7.5). 


\section{Chapter 8}

\section{Conclusions}

We have developed a robust numerical strategy for finding smooth solutions to the Dirichlet velocity boundary control of fluid-solid conjugate heat transfer problems. For this optimal control problem of multiphysics processes on multidomains, we formulated the state equations, objective function, adjoint equations and first order optimality conditions in both the strong and the weak formulations, and we also discussed the well-posedness of the continuous optimal control problem. Moreover, we studied the proper Finite Element discretization of the control problem and emphasized the importance of making the objective function consistent on both the continuous and the discrete levels, namely, imposing regularization on the control gradient on both levels. Furthermore, we studied a number of numerical optimization algorithms. The numerical smoothing technique, aiming to computationally resolve the nonsmoothness issue of the control profile, was illustrated in a variant of the Steepest Descent method and the Quasi-Newton L-BFGS method; the numerical continuation technique, which is applied to the regularization parameters, was combined with the L-BFGS method to enhance the robustness of the optimization scheme for difficult control problems, such as the case in which the initial control profile is far from optimal.

The efficiency and robustness of our solution strategy have been demonstrated via two suites of numerical test problems. In the test case of recovering the boundary control profile of Poiseuille flow, we illustrated the necessity of the regularization on the control gradient in the objective function (2.4) and of the numerical smoothing device for the control update in (6.2). We also tested a 


\section{CHAPTER 8. CONCLUSIONS}

few feasible choices on the observation operator in the objective function and proposed a strategy of choosing regularization parameters $\beta_{1}$ and $\beta_{2}$. In the second suite of numerical tests, with a low quality initial guess on the control profile, we demonstrated the robustness of the L-BFGS method first with 5-phase regularization continuation for the boundary control of the channel flow and then with 2-phase regularization continuation for the boundary control of the CHT flow past a square cylinder. In conclusion, we have generated a roadmap for providing smooth and robust solutions to optimal control of multiphysics processes. We believe our strategy is general and can be extended to other optimal control problems. 


\title{
Part III
}

\section{Parallel Scalable Solutions for}

Conjugate Heat Transfer Models in

Simulations of Reactor Thermal

\author{
Hydraulics
}




\section{Chapter 9}

\section{Parallel Scalable Preconditioners for Multiphysics Simulations of CHT}

\section{processes}

\subsection{Overview}

Solving a linear system

$$
A x=b,
$$

invites two approaches, namely direct solvers and iterative solvers. Although direct solvers such as LU decomposition are robust on solving the nonsingular though generally ill-conditioned linear systems generated by the discretization of PDEs, iterative methods are most often the favorite choice for linear equations involving a large number of variables in high dimensions. This is due to their great advantages of memory and per-iteration implementation scalability. For an estimate on the memory consumption and the computational complexity of direct methods, see [59].

However, for iterative methods to be effective, including both the stationary and nonstationary iterative methods, the coefficent matrix $A$ needs have a condition number close to one, or more generally, the eigenvalues of the matrix $A$ need to form a small number of tight clusters $[40,113]$. 
Otherwise, a preconditioner is necessary to accelerate the convergence of the iterative solution process. We illustrate the key idea of preconditioning using a stationary iterative method, Richardson method. Suppose that $x^{\star}$ is the exact solution of (9.1) and $M$ is a left-preconditioner matrix for the matrix $A$, then we have the preconditioned linear system

$$
M \cdot A x=M \cdot b .
$$

Applying the Richardson iteration at the $k$-th iterate $x^{k}$ to (9.2), we have the following derivation

$$
\begin{aligned}
x^{k+1} & =x^{k}+M\left(b-A x^{k}\right) \\
e^{k+1} & =e^{k}+M\left(-A e^{k}\right) \\
e^{k+1} & =(I-M A) e^{k},
\end{aligned}
$$

where we have defined the iteration error at the $k$-th step as $e^{k}:=x^{k}-x^{\star}$. It is evident that when $M A$ is a better approximation to the identity matrix, or when $M$ is a better approximation of $A^{-1}$, the convergence rate of (9.3) will improve. The same result holds for CG, GMRES and other nonstationary iterative methods from the family of Krylov subspace iterations.

The following sections introduce the strategy of choosing effective iterative methods for different applications. We discuss scalable preconditioners for diffusion and convection-diffusion equations in Section 9.2, for Stokes and Navier-Stokes equations in Section 9.3.

\subsection{Preconditioners for Diffusion and Convection-Diffusion Equations}

The discrete equation (3.4) resulting from the Finite Element discretization of the diffusion equation (3.1) is generally symmetric positive definite (SPD) when the Dirichlet boundary nodes are treated properly. The conjugate gradient (CG) method is a very effective iterative method for such systems [113]. Moreover, as a member of the Krylov solvers family, the CG method also enjoys the ease of parallelization, which comes naturally from its major component, namely, matrix-vector multiplications. Only when load balance is difficult to maintain between the inner product synchronizations of CG, do we have issues of maintaining distributed memory parallel scalability. Furthermore, effective parallel preconditioners, such as Domain Decomposition (DD) [88, 91, 119, 123], 
Geometric Multigrid (GMG), and Algebraic Multigrid (AMG) $[33,112,124]$ can greatly shrink the spectral radius of the overall preconditioned coefficient matrix and accelerate the convergence of CG. For discretizations that do not store the grid hierarchy, AMG is a suitable choice and has shown good scalability for the diffusion equation in parallel [8] .

The discrete equation (3.11) resulting from the Finite Element discretization of the convectiondiffusion equation (3.5) is always nonsymmetric when the wind $\mathbf{w}$ is nonzero. The CG method is not suitable for nonsymmetric systems. Suitable choices from the Krylov family are biconjugate gradient method (BiCG), biconjugate gradient stabilized (BiCGSTAB) [125], quasi-minimal residual method (QMR) [56], transpose-free QMR method (TFQMR) [55], and the generalized minimal residual method (GMRES) [114]. Both Geometric Multigrid (GMG) and Algebraic Multigrid (AMG) methods can be used as preconditioners inside the Krylov iterations to solve convectiondiffusion equations $[106,129]$. When the GMG is applied, both the fluid direction and the instability of the discretization on the coarse grid and have to be properly treated [48].

\subsection{Preconditioners for Stokes and Navier-Stokes Equations}

In this section, we review the preconditioners for Stokes and Navier-Stokes equations. Preconditioning is an essential technique in solving discrete linear systems arising from the discretization of incompressible fluid systems. Our treatment follows that of Elman et al. [48].

\subsubsection{Stokes Equations}

When the viscosity $v$ in the equation (3.13) is very large, the advective inertial force is very small compared to the viscous forces, and the flow is creeping. After neglecting the quadratic inertial term $(\mathbf{u} \cdot \nabla) \mathbf{u}$ in (3.13), we write the Stokes equations

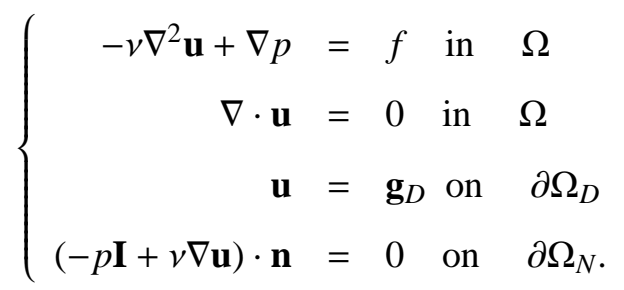


Using the trial and test function spaces in Section 3.3 and the stable Q2-Q1 discretization for the velocity-pressure pair, we obtain the discrete system

$$
\left(\begin{array}{ccc}
A & 0 & B_{x}^{T} \\
0 & A & B_{y}^{T} \\
B_{x} & B_{y} & 0
\end{array}\right)\left(\begin{array}{c}
\delta \mathbf{u}_{x} \\
\delta \mathbf{u}_{y} \\
\delta p
\end{array}\right)=\left(\begin{array}{c}
\mathbf{R}_{x} \\
\mathbf{R}_{y} \\
\mathbf{r}
\end{array}\right),
$$

with

$$
A=\left[a_{i j}\right], a_{i j}=v \int_{\Omega} \nabla \phi_{j} \cdot \nabla \phi_{i} ; \quad B_{\alpha}=\left[b_{\alpha i j}\right], b_{\alpha i j}=-\int_{\Omega} \psi_{i} \frac{\partial \phi_{j}}{\partial \alpha},
$$

where $\alpha \in\{x, y\}$. Alternatively, stabilized elements can also be used. In that case, the $(3,3)$ position of the block matrix in equation (9.5) is a nonzero block matrix. In either of the cases with stable or with stablized elements, the coefficient matrix $\mathbf{C}$, where

$$
\mathbf{C}:=\left(\begin{array}{cc}
\mathbf{A} & \mathbf{B}^{T} \\
\mathbf{B} & 0
\end{array}\right), \mathbf{A}:=\left(\begin{array}{cc}
A & 0 \\
0 & A
\end{array}\right) \text { and } \mathbf{B}:=\left(\begin{array}{ll}
B_{x} & B_{y}
\end{array}\right),
$$

is symmetric but indefinite, namely, with both positive and negative eigenvalues [48]. Suitable choices from the Krylov family are the minimal residual (MINRES) and symmetric LQ (SYMMLQ) methods [107]. To effectively precondition the discrete Stokes matrix $\mathbf{C}$, we have to take the block structure into account. Moreover, when either MINRES or SYMMLQ is used as a solver, a symmetric preconditioner is desirable to preserve the symmetry of the preconditioned system. But this restriction is relaxed for GMRES. From the block factorization

$$
\left(\begin{array}{cc}
\mathbf{A} & \mathbf{B}^{T} \\
\mathbf{B} & 0
\end{array}\right)=\left(\begin{array}{cc}
\mathbf{I} & 0 \\
\mathbf{B A}^{-1} & \mathbf{I}
\end{array}\right)\left(\begin{array}{cc}
\mathbf{A} & 0 \\
0 & -\mathbf{B} \mathbf{A}^{-1} \mathbf{B}^{T}
\end{array}\right)\left(\begin{array}{cc}
\mathbf{I} & \mathbf{B A}^{-1} \\
0 & \mathbf{I}
\end{array}\right),
$$

it is clear that the block matrix

$$
\left(\begin{array}{cc}
\mathbf{A} & 0 \\
0 & -\mathbf{B} \mathbf{A}^{-1} \mathbf{B}^{T}
\end{array}\right)
$$

has the same spectrum as the Stokes matrix $\mathbf{C}$ in (9.7). In fact, it can be shown that when the inverse of (9.9) is used as a preconditioner, MINRES will converge in three steps [48]. However, in practice, the inversion of the Schur complement $\mathbf{S}:=-\mathbf{B} \mathbf{A}^{-1} \mathbf{B}^{T}$ is too expensive to carry out. Therefore, a 
good approximation of the Schur complement $\mathbf{S}$ is sought. For both stable and stablized elements, the pressure mass matrix $Q_{p}$ is spectrally equivalent to $S[118,126]$. In fact, when the pressure mass matrix $Q_{p}$ is used to precondition the Schur complement $S$, the preconditioned stokes system has a bounded spectrum. In summary, the block preconditioner

$$
\left(\begin{array}{cc}
\tilde{\mathbf{A}} & 0 \\
0 & Q_{p}
\end{array}\right)
$$

is effective, as long as $\tilde{\mathbf{A}}$ is a good approximation to the discrete vector Laplacian $\mathbf{A}$. Efficient parallel preconditioners for $\mathbf{A}$ include DD, GMG, AMG preconditioners, as discussed in Section 9.2. Since the mass matrix $Q_{p}$ is well conditioned, any parallel preconditioners are effective.

\subsubsection{Navier-Stokes Equations}

It is a more complex task to precondition the Navier-Stokes equation (3.20). We write the discrete Navier-Stokes system

$$
\mathbf{H}:=\left(\begin{array}{cc}
\mathbf{F} & \mathbf{B}^{T} \\
\mathbf{B} & 0
\end{array}\right) \text {, where } \mathbf{F}:=\left(\begin{array}{cc}
A+N+W_{x x} & W_{x y} \\
W_{y x} & A+N+W_{y y}
\end{array}\right) \text { and } \mathbf{B}:=\left(\begin{array}{ll}
B_{x} & B_{y}
\end{array}\right) .
$$

First, the block matrix $\mathbf{H}$ is nonsymmetric. The GMRES method from the Krylov solvers family is a suitable choice. Again, we have to take the block structure into account for effective preconditioning. Yet, there is no symmetry to preserve this time, since the coefficient matrix $\mathbf{H}$ is nonsymmetric. Based on the matrix factorization

$$
\left(\begin{array}{cc}
\mathbf{F} & \mathbf{B}^{T} \\
\mathbf{B} & 0
\end{array}\right)\left(\begin{array}{cc}
\mathbf{F} & \mathbf{B}^{T} \\
0 & -\mathbf{S}
\end{array}\right)^{-1}=\left(\begin{array}{cc}
\mathbf{I} & 0 \\
\mathbf{B F}^{-1} & \mathbf{I}
\end{array}\right)
$$

where $\mathbf{S}=\mathbf{B F}^{-1} \mathbf{B}^{T}$, an exact preconditioner would be

$$
\mathbf{P}=\left(\begin{array}{cc}
\mathbf{F} & \mathbf{B}^{T} \\
0 & -\mathbf{S}
\end{array}\right)
$$

with

$$
\mathbf{P}^{-1}=\left(\begin{array}{cc}
\mathbf{F} & \mathbf{B}^{T} \\
0 & -\mathbf{S}
\end{array}\right)^{-1}=\left(\begin{array}{cc}
\mathbf{F}^{-1} & 0 \\
0 & \mathbf{I}
\end{array}\right)\left(\begin{array}{cc}
\mathbf{I} & -\mathbf{B}^{T} \\
0 & \mathbf{I}
\end{array}\right)\left(\begin{array}{cc}
\mathbf{I} & 0 \\
0 & -\mathbf{S}^{-1}
\end{array}\right) .
$$


Here, we have the same issue as in the Stokes system, namely, the inversion of the Schur complement $\mathbf{S}$ is too expensive to carry out. Besides, the inversion of the veloctiy block $\mathbf{F}$ is also very expensive. Therefore, we seek approximations to the Schur complement $\mathbf{S}$ and the velocity block F. We denote an approximate preconditioner by

$$
\tilde{\mathbf{P}}^{-1}=\left(\begin{array}{cc}
\tilde{\mathbf{F}}^{-1} & 0 \\
0 & \mathbf{I}
\end{array}\right)\left(\begin{array}{cc}
\mathbf{I} & -\mathbf{B}^{T} \\
0 & \mathbf{I}
\end{array}\right)\left(\begin{array}{cc}
\mathbf{I} & 0 \\
0 & -\tilde{\mathbf{S}}^{-1}
\end{array}\right),
$$

where $\tilde{\mathbf{F}}$ and $\tilde{\mathbf{S}}$ are some approximations for $\mathbf{F}$ and $\mathbf{S}$.

The Krylov subspace iteration of system (3.20) with an approximate preconditioner $\tilde{\mathbf{P}}$ requires the matrix-vector multiplication of $\tilde{\mathbf{P}}^{-1}$. This in turn requires matrix-vector multiplications by $\tilde{\mathbf{F}}^{-1}$ in the velocity subspace and $\tilde{\mathbf{S}}^{-1}$ in the pressure subspace. Each of these matrix-vector multiplication can be achieved via iterative methods. Many different strategies have been studied for the preconditioning of the Schur complement $\mathbf{S}$ of Navier-Stokes equations. The precondioner $\tilde{\mathbf{S}}:=\mathbf{A}_{p} \mathbf{F}_{p}^{-1} \mathbf{M}_{p}$ [50] based on the commutator of the convection-diffusion operator $\mathbf{F}$-scaled and the gradient operator $\mathbf{B}^{T}$-scaled has shown mesh-independent and mildly $R e$-dependent convergence for steady state Picard-linearization of the Navier-Stokes equation. The boundary consistency is discussed in [48]. We expect the performance to be similar for the Newton-linearization of Navier-Stokes equations, because they share the similar block matrix structure with only different $\mathbf{F}$ blocks. Efficient choices of applying the matrix-vector multiplications of $\tilde{\mathbf{S}}^{-1}$ in parallel include multigrid methods, either geometric or algebraic. 
CHAPTER 10. PARALLEL LINEAR SOLVERS FOR SIMULATIONS OF REACTOR THERMAL

HYDRAULICS

\section{Chapter 10}

\section{Parallel Linear Solvers for Simulations of Reactor Thermal Hydraulics}

\subsection{Overview}

In this chapter, we describe research [130], which is related to the preconditioning of Navier-Stokes equations, using the Algebraic Multigrid Method (AMG). We show the scalability of the resulting parallel implementation. This work is for the Reynolds-averaged Navier-Stokes (RANS) equations with multiple fluid species (up to 5 in our context) on unstructured meshes. The Newton-Krylov machinery is carried out with specific user-defined preconditioners to capture the fine structure of the simulation. It demonstrates the robustness of the physics-based block preconditioners for complex turbulence multiphase fluid simulations based on RANS. The physical setting significantly generalizes the single fluid laminar incompressible case at the heart of our control problem; however, we have not applied control to the reactor thermal hydraulic problem. We aim here to demonstrate that the linear algebraic solvers can be extended to solve more complex fluid systems.

Performing RANS simulations of turbulent flow inside large computational domains at macroscale requires the solution of a nonlinear system of PDEs, which represents the conservation laws for multi-phase fluid [108]. For steady-state problems, discretizing the PDE on a finite volume mesh results in a set of nonlinear algebraic equations, $F(x)=0$, which can be solved using a variant 
CHAPTER 10. PARALLEL LINEAR SOLVERS FOR SIMULATIONS OF REACTOR THERMAL HYDRAULICS

of Newton's method. At iterate $x^{n}$, in order to obtain the next iterate $x^{n+1}=x^{n}+\omega \delta x^{n}$, Newton requires the correction $\delta x^{n}$ from solving a large sparse system of linear equations of the form, $A \delta x^{n}=b^{n}$, where $A=\left[a_{i j}\right]$ is the Jacobian of $F(x)$ evaluated at $x^{n}$, and $b^{n}$ is the negative of the residual of the nonlinear system, namely, $b^{n}=-F\left(x^{n}\right)$. When the underlying continuous governing system is nonlinearly stiff, $F(x)$ may contain continuation temporal terms [35], and the Newton iteration is typically damped through an under-relaxation factor $\omega$, where $0<\omega<1$. In practice, for an ill-conditioned linear system, combining a Krylov subspace method with a preconditioner is essential to reduce the condition number of the resulting coefficient matrix, tighten the clustering of the eigenvalues of the preconditioned system, alleviate the effect of round-off errors, and enhance the convergence rate. In what follows, we briefly describe two perspectives that are related to this dissertation work.

\subsection{AMG Preconditioned Newton-Krylov Methods}

Regarding the choice for preconditioner, we separate each fluid component [130], and then apply a Krylov solver object to perform the inversion of each subfield. This method can be viewed as a multiplicative composition of preconditioners built up by submatrices. In fact, the preconditioner can separate coupled degrees of freedom within each mesh cell, namely, each variable in the conservation laws. It turns out that the decoupling between the velocity and the pressure variables is critical for achieving effective preconditioning. Denoting $i$ as the index for the $i$-th fluid species, we group all the velocity components $\boldsymbol{u}_{i}$ into one field, the pressure $p_{i}$ into another field, and the volume fractions $\alpha_{i}$ into a third field. It can be represented as an index set $\left\{\left\{\boldsymbol{u}_{i}\right\},\left\{p_{i}\right\},\left\{\alpha_{i}\right\}\right\}$. This preconditioner intrinsically has an emphasis on the block structure and thus requires the matrix to be partitioned on block level over all processes. With this partitioning on all DOFs, we can then apply the AMG that is based on parallel aggressive coarsening [74] as a preconditioner for the Newton-Krylov iterations. This process can be viewed as applying a block diagonal preconditioner, where each block represents a preconditioner for the corresponding velocity variables. This work is done by using PETSc [12-14] and Hypre [7, 8, 51]. 
CHAPTER 10. PARALLEL LINEAR SOLVERS FOR SIMULATIONS OF REACTOR THERMAL HYDRAULICS

\subsection{Scalable Data Interface and Scaling Results}

Our solver has shown almost linear computational complexity with respect to the problem size $M$, which is also the dimension of the Jacobian $A$ or the overall number of degrees of freedom (DOF) on all processes. In other words, for fixed DOF per CPU core, our solver uses almost constant time as the problem size increases. Indeed, for each plain GMRES iteration, the complexity is $O(r \cdot M)$ on a sparse matrix of size $M$ with a nonzero ratio per row $r / M$. Therefore, for each preconditioned GMRES iteration of a sparse matrix of size $M$, the complexity will be the sum of $O(r \cdot M)$ and the complexity of the sparse preconditioning. In the context of using BoomerAMG as a preconditioner inside the Krylov iteration, the computational complexity of the sparse preconditioning increases almost linearly with respect to the problem size $M$ [121], that is, $O(c \cdot M)$ for some positive constant $c$. Therefore, our solver has almost linear complexity per iteration.

With a well-balanced mesh partitioner and discretization, we build up a data interface with minimal inter-processor data movement to set up the exact memory preallocation for assembling the Jacobian and to achieve the scalable performance. All these are done on the block matrix level.

In Figure 10.1, we show the weak scaling results of the full and partial-splitting-2 strategies for two-phase flow in the reactor core geometry. The weak scaling is a measure of how the solution time varies with the number of CPU cores for a fixed problem size per process. We denote by $T(M)$ the time to solve a problem of size $M$ on a single process or CPU core, and by $T(M \cdot P)$ the time to solve a problem of size $M \cdot P$ on $P$ processes, with a fixed problem size $M$ per process. A solver is scalable if the ratio $T(M \cdot P) / T(M)$ is close to one.

A series of numerical experiments have been performed on the Opteron Blade Cluster located at the Computational Center for Nanotechnology Innovations (CCNI) of RPI, Troy, NY. This cluster consists of 462 IBM LS21 blade servers with two dual-core $2.6 \mathrm{GHz}$ Opteron processors, gigabit Ethernet and Infiniband interconnects, and system memory in eight, twelve and sixteen gigabyte configurations. The computational speed improvements to the linear solvers and thus the full simulation would be used for various multiphase problems such as a loss-of-flow accident scenario in a Gen. IV reactor.

The cross-sectional view of fuel channels for a section of the assembly is shown in [130]. Thirty 
CHAPTER 10. PARALLEL LINEAR SOLVERS FOR SIMULATIONS OF REACTOR THERMAL HYDRAULICS

complete fuel rods of $8 \mathrm{~mm}$ diameter and $10.4 \mathrm{~mm}$ pitch are encompassed by the fluid domain along with several surrounding partial rods at the boundaries. The length of the domain was varied to control the aspect ratio of the elements. This assured a constant DOF per core and helped to preserve the aspect ratio of the elements. The DOF per core was fixed at $1.175 E 5$ along the weak scaling path, so that each core used same amount of memory

It was observed that, for a given run, a large fraction of the computational time has been spent on the function calls KSPSetup and KSPSolve. For the two-phase flow case, the timing on KSPSetup was 8 times larger, and on KSPSolve was 1.6 times larger, whereas the problem itself was 12 times bigger. In contrast, using the partial-splitting-2 strategy, the timing on KSPSetup was 4 times larger and on KSPSolve was 1.3 times larger. As a result, the partial-splitting-2 strategy turns out to be more scalable. This can be seen from the ratio $T(M \cdot P) / T(M)$ from Figure 10.1, in which the horizontal axis represents the number of processes $N P$, the vertical axis represents the wall time for the solution process, and connected blue and red lines represent $T(M \cdot P)$ of the full and partialsplitting-2 strategies respectively.

Furthermore, there seems to be some nonscalable component in the setting up stage of this particular preconditioner which damages the overall weak scalability, and this will be the subject of a future study. On the other hand, the observation just described has confirmed that it would be beneficial to reuse the preconditioner during a nonlinear run to avoid the expense of setting up the preconditioner at each step of the time integration, in order to achieve the overall scalability of the nonlinear solver. 
CHAPTER 10. PARALLEL LINEAR SOLVERS FOR SIMULATIONS OF REACTOR THERMAL HYDRAULICS

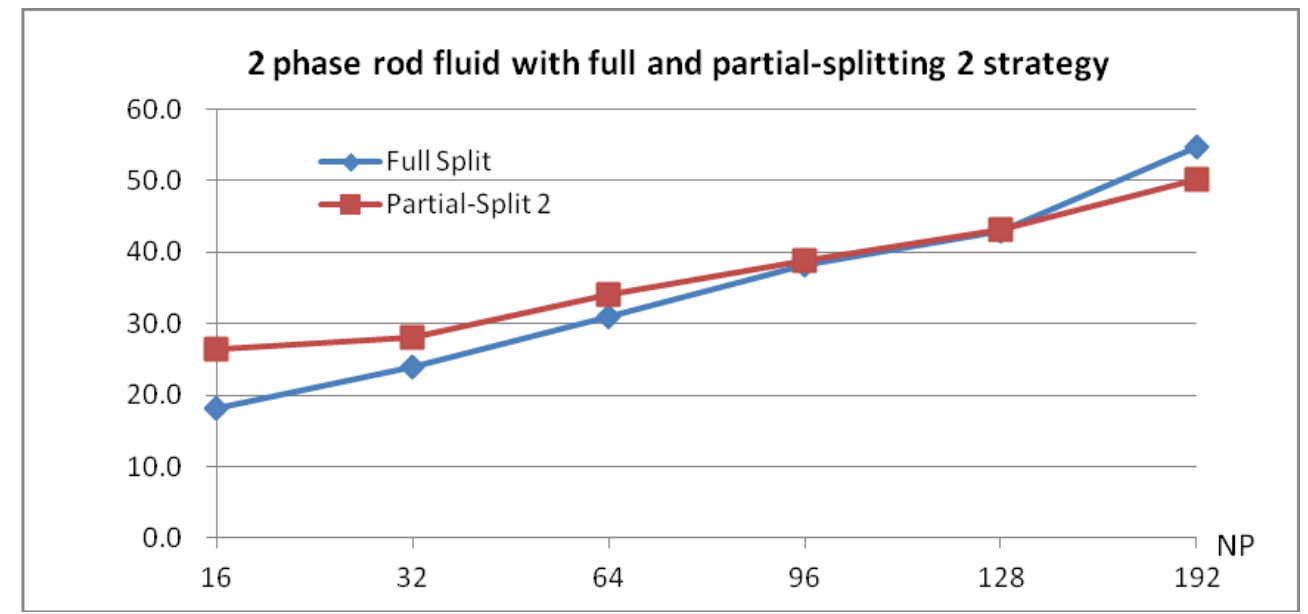

Figure 10.1: Weak scaling results with full and partial-splitting-2 strategies for two-phase flow in the reactor core geometry. 


\section{Chapter 11}

\section{Future Directions}

\subsection{Overview}

In this section, we discuss the necessary steps to achieve parallel scalable solutions to optimal control of CHT processes, and the possibilities of adapting the current optimization framework to more practical engineering settings, such as fluids in 3D complex geometry, compressible fluids, buoyancy effects, phase change, turbulence, real-time control, control with noisy measurements, and optimal control of PDEs with random coefficients.

First, to ensure the overall scalability of our control algorithms, we need investigate two aspects, namely the algorithmic scalability and the implementation scalability. In Section 11.2, we discuss effective parallel preconditioners for solving the discrete state and the discrete adjoint equations. This demonstrates, up to certain contemporary practical values for engineering computations, the algorithmic scalability of our solvers. For computations that push today's concurrency limits, or push beyond them to the exascale, there remains significant work to be done on both preconditioning, and on the Krylov accelerator, itself. See [68]. Indeed, at each optimization iteration, most of the computational cost comes from calculating the reduced gradient and line search. The former involves solving a pair of state and adjoint PDEs, and the later involves solving the state equation repeatedly. Therefore, the computational kernel in the optimal control code is solving PDEs, and its scalability mostly depends on the scalability of the PDE solvers and preconditioners. In Appendix 
$\mathrm{C}$, we focus on the algorithmic scalability of our optimizer and discuss an effective optimization algorithm, namely the sequential quadratic programming (SQP) method, which does not require exact solutions of the state or the adjoint equations and does not compromise the superlinear convergence rate. Finally, The implementation scalability can be ensured by using parallel marix-vector multiplications inside the Krylov iteration, using truncated Krylov bases, and using inexact Newton iterations in the SQP method. In Section 11.3, we discuss multiple research directions that can be explored based this thesis work.

\subsection{Scalable Preconditioners for the State Equations}

It is evident that the Finite Element matrix in (5.5) of the state equations (2.1)-(2.3) has a block structure, which assembles the discrete Navier-Stokes, discrete convection-diffusion, and discrete diffusion operators. In the segregated approach in Remark 5.2.1, the solution process of the state equations is equivalent to using the Finite Element matrix

$\left(\begin{array}{ccccc|cc}A+N+W_{x x} & W_{x y} & B_{x}^{T} & 0 & 0 & 0 & 0 \\ W_{y x} & A+N+W_{y y} & B_{y}^{T} & 0 & 0 & 0 & 0 \\ B_{x} & B_{y} & 0 & 0 & 0 & 0 & 0 \\ 0 & 0 & 0 & I & 0 & 0 & 0 \\ 0 & 0 & 0 & 0 & I & 0 & 0 \\ \hline 0 & 0 & 0 & 0 & 0 & A_{f}+N_{f} & 0 \\ 0 & 0 & 0 & 0 & 0 & 0 & A_{s}\end{array}\right)\left(\begin{array}{c}\delta u_{x} \\ \delta u_{y} \\ \delta p \\ \delta \sigma_{x} \\ \delta \sigma_{y} \\ \delta T_{f} \\ \delta T_{s}\end{array}\right)$.


From the block preconditioners for various discrete operators in Chapter 9 , we know that the following "physics-based" block preconditioner

$$
\left(\begin{array}{ccccc|cc}
\tilde{A}+\tilde{N}+\tilde{W}_{x x} & \tilde{W}_{x y} & B_{x}^{T} & 0 & 0 & 0 & 0 \\
\tilde{W}_{y x} & \tilde{A}+\tilde{N}+\tilde{W}_{y y} & B_{y}^{T} & 0 & 0 & 0 & 0 \\
0 & 0 & \tilde{S} & 0 & 0 & 0 & 0 \\
0 & 0 & 0 & I & 0 & 0 & 0 \\
0 & 0 & 0 & 0 & I & 0 & 0 \\
\hline 0 & 0 & 0 & 0 & 0 & \tilde{A}_{f}+\tilde{N}_{f} & 0 \\
0 & 0 & 0 & 0 & 0 & 0 & \tilde{A}_{s}
\end{array}\right)
$$

would be effective for the discrete CHT state matrix. Moreover, we can also build an effective preconditioner for the discrete adjoint equations due to its structure similarity to the discrete state equations. With an effective preconditioner, the preconditioned matrix has a spectrum that is only weakly dependent on the problem size, and thus the preconditioned Krylov subspace iteration is algorithmic scalable [25,49]. Moreover, with a parallel scalable implementation $[13,75]$ of the Krylov subspace iteration method, the implementation scalability can be ensured.

\subsection{Adaptation of the Optimization Framework to Practical Engi- neering Settings}

As we have discussed in the Chapter 1, the computational kernel in the optimal control code is solving the state and the adjoint PDEs. Moreover, it is evident from Chapter 10 that parallel scalable PDE solvers based on the Newton-Krylov-AMG framework have shown good scalability for two-phase turbulent fluids in 3D complex reactor core geometry. Therefore, our simulation-based optimal control strategy can be carried out in these correspondingly more realistic settings. However, many engineering codes are using various discretizations other than FEM, which makes it difficult to obtain the analytical Jacobian of the state equations. A remedy to this is to use automatic differentiation tools [101] to compute the approximated Jacobian for both the state and the adjoint equations. Alternatively, the Jacobian-Free Newton-Krylov framework [93] is also applicable. In 


\section{CHAPTER 11. FUTURE DIRECTIONS}

this case, the "optimize then discretize" approach is suitable, and special care has to be taken in implementing the boundary conditions of the adjoint equations.

In our CHT control model, we have currently considered incompressible viscous coolant fluids without buoyancy effects. But when the temperature change of the fluid has a large impact on its density, the thermal expansion inside the fluid cannot be neglected and we need consider the Boussinesq approximation [95], which couples the temperature with the velocity in the momentum transport equations. In this case, we need treat the velocity-temperature coupling terms in both the state and the adjoint equations.

Moreover, for compressible coolant fluids governed by the "full" Navier-Stokes equations, which can be described in conservation forms as a hyperbolic system, the resulting optimal control problems have a similar structure as those appear in the aerodynamic shape optimization. Scalable solvers for compressible fluids are studied in [61].

In almost all practical settings for conjugate heat transfer processes, the flow is turbulent [18]. PDE-based modeling of Turbulence is to solve the Reynolds-averaged Navier-Stokes Equations (RANS), which are derived by introducing zero-mean fluctuations to average fluid properties. We introduce the Reynolds decomposition for the velocity, pressure, and temperature variables

$$
\begin{aligned}
& \mathbf{u}:=\mathbf{U}+\mathbf{u}^{\prime} \\
& \mathbf{p}:=\mathbf{P}+\mathbf{p}^{\prime} \\
& T:=\mathbf{T}+T^{\prime},
\end{aligned}
$$

where terms in capital and bold letters represent the time average of fluid properties and terms with primes ' represent the fluctuations with zero means. Using the Reynolds decomposition (11.3) and the equations (2.1)-(2.2), we have the RANS for incompressible Newtonian fluids

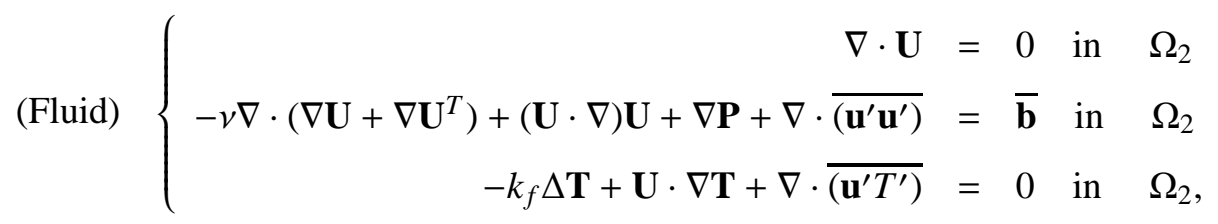

where $\bar{A}$ represent the time average of $A$. In (11.4), the turbulent stresses $\overline{\mathbf{u}^{\prime} \mathbf{u}^{\prime}}$ and turbulent fluxes $\overline{\mathbf{u}^{\prime} T^{\prime}}$ are new unknowns and depend on the turbulent flow properties. Therefore, to model the 


\section{CHAPTER 11. FUTURE DIRECTIONS}

turbulent stresses and turbulent fluxes and to bring (11.4) to closure, we need additional equations [127], such as zero equation, one equation, and two equation models. It is evident that the turbulence model (11.4) is much more complex with additional unknowns and additional equations than the laminar model (2.1)-(2.2); therefore, the derivation of the optimality condition for optimal control of systems governed by (11.4) is more involved. We also note that when the underlying discretization of (11.4) does not allow us to obtain the analytical Jacobian of the state equations, we can use automatic differentiation tools [101] to compute the approximated Jacobian for both the state and the adjoint equations.

Furthermore, it is very common that real-time controls are desirable in engineering practice [20]. In these cases, both the constraints and the objective are time dependent, and the resulting adjoint equations are also time dependent. At each optimization iteration, we need to solve both the state and the adjoint equations on the whole time domain to obtain the reduced gradient, and this renders the full space SQP approach impractical, due to its excessive memory usage when simultaneously solving nonlinear implicit systems coupled in different intervals in time and multiphysics in space. Reduced SQP is suitable, and parallelism on the temporal level can be utilized, such as the parareal methods $[11,20]$.

In practice, target measurements are very often subject to noise. Specialized variational formulations have been derived to deal with noisy data. See [9] for the solution to the parameter identification problem based on noisy measurements of the continuum deformations of a non-homogeneous isotropic solid.

We have also noticed a recent surge in interest and research efforts in optimal control of PDEs with random coefficients, due to that many of the simulation parameters cannot be measured exactly and very often these measurements are subject to uncertainty. Examples of such parameters are the Reynolds number, thermal diffusivity, density, shape geometry, and boundary conditions [6]. These problems have proven computationally very challenging, since now we need to solve many optimal control problems in the sampling space of the infinite dimensional probability space. However, the efficiency of the control algorithms in these cases still lies in the efficiency of the PDE solvers, since the computational kernel in optimal control for PDEs with random coefficients is still solving 


\section{CHAPTER 11. FUTURE DIRECTIONS}

PDEs. To solve PDEs with random coefficients, there are two class of numerical methods, namely projection-based methods and sample-based methods. The former includes the polynomial chaos and stochastic Galerkin methods, and the latter includes the Monte Carlo and stochastic collocation methods [94]. It would be a great interest to extend our current work to optimal control of CHT with random coefficients. 


\section{Part IV}

\section{Conclusions}




\section{Chapter 12}

\section{Conclusions}

Our original objective of this work is to build up an optimal control model for CHT processes using first principles, since the efficiency of the current forced convection heat transfer systems are far from optimal due to either over-simplified mathematical formulations or vague safety estimates. Our motivations for this research lie in the fact that $\mathrm{CHT}$ control models have many important industrial applications and great potential to improve the thermal efficiency of current cooling systems and to reduce the amount of coolant fluid in the system. However, such a CHT control model does not exist in literature.

As a consequence of our research, we have built up, analyzed and implemented an Dirichlet boundary control model for CHT processes based on first principles, namely, the conservation of mass, momentum, and energy. We have also demonstrated a strategy for smooth and robust solutions for Dirichlet boundary control of fluid-solid conjugate heat transfer processes. Also, we have demonstrated the effectiveness and scalability of the "physics-based" block preconditioners for multiphase fluid turbulence simulations based on Navier-Stokes equations. Finally, based on the research results that we have shown, a roadmap can be extrapolated for parallel scalable solutions for Dirichlet boundary control of fluid-solid conjugate heat transfer processes, and this is the future research direction.

A summary for each chapter of the dissertation is as follows. In Part I Chapter 2, we derived the mathematical formulation of the optimal control problem and its underlying physical constraints, 


\section{CHAPTER 12. CONCLUSIONS}

namely the governing PDEs that describe the CHT process on a simplified fluid-solid domain. In Chapter 3, we discussed the building blocks of the finite element modeling for each single physics involved in the CHT process.

In Part II, we demonstrated an framework for generating smooth and robust boundary control profiles. In Chapter 4, we set up the variational formulation for the state equations, derived the first order optimality conditions and discussed the well-posedness of the control problem in infinite dimensional function spaces. In Chapter 5, we studied the finite element assembly of the state equations, explained the nonsmoothness of the boundary control profile on both the continuous and the discrete levels using regularity estimates, and emphasized the importance of keeping the regularity term on the control gradient in both the continuous and the discrete objective functions. We illustrated in Chapter 6 the numerical smoothing technique that resolved the nonsmoothness issue in the discrete optimization schemes, and the numerical continuation technique on regularization parameters that circumvented the difficulty of locating the global minimum in one shot when the initial guess of the control problem was far from optimal. In Chapter 7, we demonstrated the feasibility, effectiveness, and robustness of our numerical optimization schemes via two suites of numerical test problems.

In Part III, we discussed the strategy for generating scalable and parallel algorithms for CHT models in Simulations of Reactor Thermal Hydraulics. In Chapter 9, we studied parallel scalable preconditioned Krylov subspace iterations for each single physics involved in the CHT procss. In Chapter 10, we summarized salient algebraic features of our work published elsewhere, which demonstrates the effectiveness and scalability of the physics-based block preconditioners for simulations of multiphase turbulent channel flows in reactor thermal hydraulics. In Chapter 11, a physicsbased block preconditioner is suggested for the state equations of the optimal control problem, and this is one of our future research directions. 


\section{Part V}

\section{Bibliography}




\section{Bibliography}

[1] F. Abergel and R. Temam, On some control problems in fluid mechanics, Theoretical and Computational Fluid Dynamics, 1 (1990), pp. 303-325.

[2] F. Abraham, M. Behr, and M. Heinkenschloss, The effect of stabilization in finite element methods for the optimal boundary control of the Oseen equations, Finite Elements in Analysis and Design, 41 (2004), pp. 229-251.

[3] V. Akçelik, G. Biros, O. Ghattas, J. Hill, D. Keyes, and B. Van Bloemen Wanders, Parallel algorithms for PDE-constrained optimization and application to optimal control of viscous flows, in Frontiers of Parallel Computing, M. Heroux, P. Raghaven, and H. Simon, eds., SIAM, Philadelphia, 2006.

[4] W. Alt and K. Malanowski, The Lagrange-Newton method for nonlinear optimal control problems, Computational Optimization and Applications, 2 (1993), pp. 77-100.

[5] I. BABUŠKa, The finite element method with Lagrangian multipliers, Numerische Mathematik, 20 (1973), pp. 179-192.

[6] I. BabušKa, F. Nobile, and R. Tempone, A Stochastic Collocation Method for Elliptic Partial Differential Equations with Random Input Data, SIAM Review, 52 (2010), pp. 317-355.

[7] A. Baker, R. Falgout, T. Gamblin, T. Kolev, M. Schulz, and U. Yang, Scaling algebraic multigrid solvers: On the road to exascale, in Competence in High Performance Computing 2010, C. Bischof, H.-G. Hegering, W. E. Nagel, and G. Wittum, eds., Springer Berlin Heidelberg, 2012, pp. 215-226. 


\section{BIBLIOGRAPHY}

[8] A. H. Baker, R. D. Falgout, T. V. Kolev, and U. M. Yang, Scaling hypre's multigrid solvers to 100,000 cores, in High Performance Scientific Computing: Algorithms and Applications, M. W. Berry, K. A. Gallivan, E. Gallopoulos, A. Grama, B. Philippe, Y. Saad, and F. Saied, eds., Springer, London, 2004, pp. 261-279.

[9] G. Bal, C. Bellis, S. Imperiale, and F. Monard, Reconstruction of constitutive parameters in isotropic linear elasticity from noisy full-field measurements, Inverse Problems, 30 (2014), p. 125004.

[10] G. BAL AND K. REN, Reconstruction of singular surfaces by shape sensitivity analysis and level set methods, Mathematical Models and Methods in Applied Sciences, 16 (2006), pp. $1347-$ 1473.

[11] G. Bal and Q. Wu, Symplectic parareal, in Domain Decomposition Methods in Science and Engineering XVII, U. Langer, M. Discacciati, D. Keyes, O. Widlund, and W. Zulehner, eds., vol. 60 of Lecture Notes in Computational Science and Engineering, Springer Berlin Heidelberg, 2008, pp. 401-408.

[12] S. Balay, J. Brown, K. Buschelman, V. Eujhout, W. D. Gropp, D. Kaushik, M. G. Knepley, L. C. McInNes, B. F. Smith, And H. Zhang, PETSc users manual, Tech. Report ANL-95/11 Revision 3.4, Argonne National Laboratory, 2013.

[13] S. Balay, J. Brown, K. Buschelman, W. D. Gropp, D. Kaushik, M. G. Knepley, L. C. Mcinnes, B. F. Smith, and H. Zhang, PETSc Web page, 2013. http://www.mcs.anl.gov/petsc.

[14] S. Balay, W. D. Gropp, L. C. McInNes, and B. F. Smith, Efficient management of parallelism in object oriented numerical software libraries, in Modern Software Tools in Scientific Computing, E. Arge, A. M. Bruaset, and H. P. Langtangen, eds., Birkhäuser Press, 1997, pp. $163-$ 202.

[15] W. Bangerth, R. Hartmann, and G. Kanschat, deal.II - a general purpose object oriented finite element library, ACM Transactions on Mathematical Software, 33 (2007), pp. 24/1$24 / 27$. 


\section{BIBLIOGRAPHY}

[16] R. Becker AND M. BRAack, A finite element pressure gradient stabilization for the Stokes equations based on local projections, Calcolo, 38 (2001), pp. 173-199.

[17] R. Becker and B. Vexler, Optimal Control of the Convection-diffusion Equation Using Stabilized Finite Element Methods, Numerische Mathematik, 106 (2007), pp. 349-367.

[18] A. Bejan, Convection Heat Transfer, Wiley, Hoboken, 3rd ed., 2004.

[19] M. Benzi, E. Haber, and L. Taralli, A Preconditioning Technique for a Class of PDEconstrained Optimization Problems, Advances in Computational Mathematics, 35 (2011), pp. 149-173.

[20] L. Biegler, O. Ghattas, M. Heinkenschloss, D. Keyes, and B. Van Bloemen Waanders, eds., Real-Time PDE-Constrained Optimization, Society for Industrial and Applied Mathematics, Philadephia, 2007.

[21] L. Biegler, O. Ghattas, M. Heinkenschloss, and B. Van Bloemen Waanders, Large-scale PDE-constrained Optimization, vol. 30 of Lecture Notes in Computational Science and Engineering, Springer, Berlin, 2003.

[22] L. Biegler, J. Nocedal, and C. Schmid, A Reduced Hessian Method for Large-Scale Constrained Optimization, SIAM Journal on Optimization, 5 (1995), pp. 314-347.

[23] L. T. Biegler, Nonlinear Programming, Society for Industrial and Applied Mathematics, Philadephia, 2010.

[24] G. BIRos AND O. GhatTAS, Inexactness Issues in the Lagrange-Newton-Krylov-Schur Method for PDE-constrained Optimization, in Large-Scale PDE-Constrained Optimization, L. Biegler, M. Heinkenschloss, O. Ghattas, and B. Bloemen Waanders, eds., vol. 30 of Lecture Notes in Computational Science and Engineering, Springer Berlin Heidelberg, 2003, pp. 93-114. 


\section{BIBLIOGRAPHY}

[25] G. Biros and O. Ghattas, Parallel Lagrange-Newton-Krylov-Schur Methods for PDEConstrained Optimization. Part I: The Krylov-Schur Solver, SIAM Journal on Scientific Computing, 27 (2005), pp. 687-713.

[26] _- Parallel Lagrange-Newton-Krylov-Schur Methods for PDE-Constrained Optimization. Part II: The Lagrange-Newton Solver and Its Application to Optimal Control of Steady Viscous Flows, SIAM Journal on Scientific Computing, 27 (2005), pp. 714-739.

[27] P. T. Boggs and J. W. Tolle, Sequential Quadratic Programming, Acta Numerica, 4 (1995), pp. 1-51.

[28] A. Borzì and V. Schulz, Computational Optimization of Systems Governed by Partial Differential Equations, Computational science and engineering series, Society for Industrial and Applied Mathematics, Philadelphia, 2011.

[29] S. Boyd and L. Vandenberghe, Convex Optimization, Cambridge University Press, New York, 2004.

[30] M. Braack and E. Burman, Local Projection Stabilization for the Oseen Problem and Its Interpretation As a Variational Multiscale Method, SIAM Journal on Numerical Analysis, 43 (2006), pp. 2544-2566.

[31] S. Brenner and R. Scott, The Mathematical Theory of Finite Element Methods, Texts in Applied Mathematics, Springer, New York, 2008.

[32] M. Breuer, J. Bernsdorf, T. Zeiser, AND F. Durst, Accurate computations of the laminar flow past a square cylinder based on two different methods: lattice-Boltzmann and finite-volume, International Journal of Heat and Fluid Flow, 21 (2000), pp. 186 - 196.

[33] W. L. Briggs, V. E. Henson, and S. F. McCormick, A Multigrid Tutorial, Society for Industrial and Applied Mathematics, Philadelphia, 2nd ed., 2000.

[34] X. CAI ANd D. Keyes, Nonlinearly Preconditioned Inexact Newton Algorithms, SIAM Journal on Scientific Computing, 24 (2002), pp. 183-200. 


\section{BIBLIOGRAPHY}

[35] T. Coffey, C. Kelley, and D. Keyes, Pseudotransient Continuation and DifferentialAlgebraic Equations, SIAM Journal on Scientific Computing, 25 (2003), pp. 553-569.

[36] S. Coluis And M. Heinkenschloss, Analysis of the Streamline Upwind/Petrov Galerkin method applied to the solution of optimal control problems, Tech. Report CAAM TR02-01, Rice University, 2002.

[37] D. Colton and R. KRess, Inverse acoustic and electromagnetic scattering theory, SpringerVerlag New York, Inc., 3rd ed., 2013.

[38] R. Dembo, S. Eisenstat, and T. Steihaug, Inexact Newton Methods, SiAM Journal on Numerical Analysis, 19 (1982), pp. 400-408.

[39] R. Dembo and T. Steinaug, Truncated-Newton algorithms for large-scale unconstrained optimization, Mathematical Programming, 26 (1983), pp. 190-212.

[40] J. W. Demmel, Applied Numerical Linear Algebra, Society for Industrial and Applied Mathematics, Philadelphia, 1997.

[41] J. Dennis, M. El-Alem, And M. Maciel, A global convergence theory for general trust-regionbased algorithms for equality constrained optimization, SIAM Journal on Optimization, 7 (1997), pp. 177-207.

[42] J. Dennis, M. Heinkenschloss, and L. Vicente, Trust-Region Interior-Point SQP Algorithms for a Class of Nonlinear Programming Problems, SIAM Journal on Control and Optimization, 36 (1998), pp. 1750-1794.

[43] J. Dennis, M. Heinkenschloss, and L. Vicente, Trice web page. http://www. caam. rice. edu/ trice/applications/cavity/cavity.html, 2013. Accessed: 2013-05-17.

[44] S. Eisenstat and H. Walker, Globally Convergent Inexact Newton Methods, SIAM Journal on Optimization, 4 (1994), pp. 393-422.

[45] _ Choosing the Forcing Terms in an Inexact Newton Method, SIAM Journal on Scientific Computing, 17 (1996), pp. 16-32. 


\section{BIBLIOGRAPHY}

[46] H. Elman, M. Mihajlović, and D. Silvester, Fast iterative solvers for buoyancy driven flow problems, Journal of Computational Physics, 230 (2011), pp. 3900-3914.

[47] H. Elman, A. Ramage, and D. Silvester, Algorithm 866: IFISS, a Matlab toolbox for modelling incompressible flow, ACM Transactions on Mathematical Software, 33 (2007), pp. 214.

[48] H. Elman, D. Silvester, and A. Wathen, Finite Elements and Fast Iterative Solvers: with Applications in Incompressible Fluid Dynamics: with Applications in Incompressible Fluid Dynamics, Oxford University Press, Oxford, 2005.

[49] H. C. Elman, V. E. Howle, J. N. Shadid, and R. S. Tuminaro, A parallel block multi-level preconditioner for the 3D incompressible Navier-Stokes equations, Journal of Computational Physics, 187 (2003), pp. 504-523.

[50] H. C. Elman, D. J. Silvester, And A. J. Wathen, Performance and analysis of saddle point preconditioners for the discrete steady-state Navier-Stokes equations, Numerische Mathematik, 90 (2002), pp. 665-688.

[51] R. Falgout And U. YAng, Hypre: A library of high performance preconditioners, in Computational Science ICCS 2002, P. Sloot, A. Hoekstra, C. Tan, and J. Dongarra, eds., vol. 2331 of Lecture Notes in Computer Science, Springer Berlin Heidelberg, 2002, pp. 632-641.

[52] B. Fischer, A. Ramage, D. J. Silvester, and A. J. Wathen, On parameter choice and iterative convergence for stabilised discretisations of advection-diffusion problems, Computer Methods in Applied Mechanics and Engineering, 179 (1999), pp. 179-195.

[53] R. Fletcher, The Sequential Quadratic Programming Method, in Nonlinear Optimization, G. Di Pillo and F. Schoen, eds., Lecture Notes in Mathematics, Springer Berlin Heidelberg, 2010, pp. 165-214.

[54] R. Fontecilla, On inexact quasi-Newton methods for constrained optimization, in Numerical Optimization 1984, Proceedings of the SIAM Conference on Numerical Optimization, SIAM, Philadelphia, 1985, pp. 102-118. 


\section{BIBLIOGRAPHY}

[55] R. Freund, A Transpose-Free Quasi-Minimal Residual Algorithm for Non-Hermitian Linear Systems, SIAM Journal on Scientific Computing, 14 (1993), pp. 470-482.

[56] R. Freund and N. Nachtigal, QMR: a quasi-minimal residual method for non-Hermitian linear systems, Numerische Mathematik, 60 (1991), pp. 315-339.

[57] O. GhatTAS AND J. H. BaRK, Optimal control of two- and three-dimensional incompressible Navier-Stokes flows, Journal of Computational Physics, 136 (1997), pp. 231-244.

[58] V. Girault and P. Raviart, Finite Element Methods for Navier-Stokes Equations: Theory and Algorithms, Springer Series in Computational Mathematics, Springer Berlin Heidelberg, 1986.

[59] G. H. Golub and C. F. Van Loan, Matrix Computations, Johns Hopkins University Press, Baltimore, 3rd ed., 1996.

[60] P. M. Gresho, Incompressible fluid dynamics: Some fundamental formulation issues, Annual Review of Fluid Mechanics, 23 (1991), pp. 413-453.

[61] W. Gropp, D. Keyes, L. C. McInnes, and M. D. Tidriri, Globalized Newton-Krylov-Schwarz Algorithms and Software for Parallel Implicit CFD, International Journal of High Performance Computing Applications, 14 (2000), pp. 102-136.

[62] M. Gunzburger, Perspectives in Flow Control and Optimization, Advances in Design and Control, Society for Industrial and Applied Mathematics, Philadephia, 2003.

[63] M. Gunzburger and H. Lee, Analysis, approximation, and computation of a coupled solid/fluid temperature control problem, Computer Methods in Applied Mechanics and Engineering, 118 (1994), pp. 133-152.

[64] M. D. Gunzburger, L. Hou, and T. P. Svobodny, Analysis and finite element approximation of optimal control problems for the stationary Navier-Stokes equations with distributed and Neumann controls, Mathematics of Computation, 57 (1991), pp. 123-151. 


\section{BIBLIOGRAPHY}

[65] M. D. Gunzburger, L. S. Hou, And T. P. Svobodny, Analysis and finite element approximation of optimal control problems for the stationary Navier-Stokes equations with Dirichlet controls, RAIRO Modélisation Mathématique et Analyse Numérique, 25 (1991), pp. 711-748.

[66] M. D. Gunzburger And S. L. Hou, Treating inhomogeneous essential boundary conditions in finite element methods and the calculation of boundary stresses, SIAM Journal on Numerical Analysis, 29 (1992), pp. 390-424.

[67] Y. HE AND D. KeYEs, Reconstructing parameters of the Fitzhugh-Nagumo system from boundary potential measurements, Journal of Computational Neuroscience, 23 (2007), pp. 251264.

[68] Y. He AND D. KeYes, Large-scale parameter extraction in electrocardiology models through Born approximation, Inverse Problems, 29 (2013), p. 015001.

[69] M. Heinkenschloss, Projected Sequential Quadratic Programming Methods, SIAM Journal on Optimization, 6 (1996), pp. 373-417.

[70] M. Heinkenschloss, Formulation and analysis of a sequential quadratic programming method for the optimal Dirichlet boundary control of Navier-Stokes flow, in Optimal Control, vol. 15 of Applied Optimization, Springer US, 1998, pp. 178-203.

[71] M. HeInKEnschloss AND L. Vicente, Analysis of Inexact Trust-Region SQP Algorithms, SIAM Journal on Optimization, 12 (2002), pp. 283-302.

[72] M. Heinkenschloss and L. N. Vicente, An interface between optimization and application for the numerical solution of optimal control problems, ACM Transactions on Mathematical Software, 25 (1999), pp. 157-190.

[73] W. D. Henshaw AND K. K. Chand, A composite grid solver for conjugate heat transfer in fluid-structure systems, Journal of Computational Physics, 228 (2009), pp. 3708-3741.

[74] V. Henson and U. YAng, BoomerAMG: a parallel algebraic multigrid solver and preconditioner, Applied Numerical Mathematics, 41 (2000), pp. 155-177. 


\section{BIBLIOGRAPHY}

[75] M. A. Heroux, R. A. Bartlett, V. E. Howle, R. J. Hoekstra, J. J. Hu, T. G. Kolda, R. B. Lehoucq, K. R. Long, R. P. Pawlowski, E. T. Phipps, A. G. Salinger, H. K. Thornquist, R. S. Tuminaro, J. M. Willenbring, A. Williams, and K. S. Stanley, An overview of the Trilinos project, ACM Transactions on Mathematical Software, 31 (2005), pp. 397-423.

[76] M. Hinze, R. Pinnau, M. Ulbrich, and S. Ulbrich, Optimization with PDE Constraints, Mathematical modelling-theory and applications, Springer, New York, 2009.

[77] K. H. Hoffman and L. Jiang, Optimal control of a phase field model for solidification, Numerical Functional Analysis and Optimization, 13 (1992), p. 1127.

[78] R. Hoppe, C. Linsenmann, and H. Antil, Adaptive Path Following Primal Dual Interior Point Methods for Shape Optimization of Linear and Nonlinear Stokes Flow Problems, in LargeScale Scientific Computing, I. Lirkov, S. Margenov, and J. Waśniewski, eds., vol. 4818 of Lecture Notes in Computer Science, Springer Berlin Heidelberg, 2008, pp. 259-266.

[79] L. S. Hou and S. S. RavindRan, Numerical approximation of optimal flow control problems by a penalty method: error estimates and numerical results, SIAM Journal on Scientific Computing, 20 (1999), pp. 1753-1777.

[80] T. Hughes and A. Brooks, A multidimensional upwind scheme with no crosswind diffusion, Finite Element Methods for Convection Dominated Flows, Applied Mechanics Division (ASME), 34 (1979), pp. 19-35.

[81] F.-N. Hwang and X.-C. CAI, A parallel nonlinear additive Schwarz preconditioned inexact Newton algorithm for incompressible Navier-Stokes equations, Journal of Computational Physics, 204 (2005), pp. 666-691.

[82] H. JäGER AND E. SACHS, Global convergence of inexact reduced SQP methods, Optimization Methods and Software, 7 (1997), pp. 83-110.

[83] A. Jameson, Advances in Aerodynamic Shape Optimization, in Computational Fluid Dynamics 2004, C. Groth and D. Zingg, eds., Springer Berlin Heidelberg, 2006, pp. 687-698. 


\section{BIBLIOGRAPHY}

[84] A. Jameson And S. Kiм, Reduction of the Adjoint Gradient Formula in the Continuous Limit, in AIAA paper, 41 st AIAA Aerospace Sciences Meeting, 2003, AIAA, 2003, pp. 16-52.

[85] A. Jameson and J. Vassberg, Studies of Alternative Numerical Optimization Methods Applied to the Brachistochrone Problem, in OptiCon 99 Conference, Newport Beach, CA, October 1999, Computational Fluid Dynamics Journal, 2000, pp. 281-296.

[86] L. John And O. Steinach, Optimal Dirichlet boundary control for the Navier-Stokes equations. Poster at the SIAM Conference on Computational Science and Engineering, Boston, Feb 2013. http://www.numerik.math.tu-graz.ac.at/ john/poster_ boston_john.pdf.

[87] R. Joslin, M. Gunzburger, R. Nicolaides, G. Erlebacher, and M. Hussaini, Self-contained automated methodology for optimal flow control, AIAA Journal, 35 (1997), pp. 816-824.

[88] R. Katz, M. Knepley, B. Smith, M. Spiegelman, and E. Coon, Numerical simulation of geodynamic processes with the Portable Extensible Toolkit for Scientific Computation, Physics of the Earth and Planetary Interiors, 163 (2007), pp. 52-68.

[89] M. D. Kelleher AND K.-T. YANG, A steady conjugate heat transfer problem with conduction and free convection, Applied Scientific Research, 17 (1967), pp. 249-269.

[90] C. Kelley and D. Keyes, Convergence Analysis of Pseudo-Transient Continuation, SIAM Journal on Numerical Analysis, 35 (1998), pp. 508-523.

[91] D. E. Keyes, How scalable is domain decomposition in practice?, in Proceedings of the 11th International Conference on Domain Decomposition Methods, Domain Decomposition Press, 1998, Bergen, Norway, 1998, pp. 286-297.

[92] B. S. Kirk, J. W. Peterson, R. H. Stogner, and G. F. Carey, libMesh: A C++ Library for Parallel Adaptive Mesh Refinement/Coarsening Simulations, Engineering with Computers, 22 (2006), pp. 237-254. http://dx. doi .org/10 . 1007/s00366-006-0049-3. 


\section{BIBLIOGRAPHY}

[93] D. A. Knoll and D. E. KeYEs, Jacobian-free Newton-Krylov methods: a survey of approaches and applications, Journal of Computational Physics, 193 (2004), pp. 357-397.

[94] D. Kouri, M. Heinkenschloss, D. Ridzal, and B. van Bloemen Waanders, A Trust-Region Algorithm with Adaptive Stochastic Collocation for PDE Optimization under Uncertainty, SIAM Journal on Scientific Computing, 35 (2013), pp. A1847-A1879.

[95] P. Kundu And I. Cohen, Fluid Mechanics, Academic Press, Burlington, 4th ed., 2007.

[96] J. L. Lions, Optimal Control of Systems Governed by Partial Differential Equations, Springer Berlin Heidelberg, 1971.

[97] A. LogG, K.-A. Mardal, and G. N. Wells, eds., Automated Solution of Differential Equations by the Finite Element Method, vol. 84 of Lecture Notes in Computational Science and Engineering, Springer Berlin Heidelberg, 2012.

[98] H. MatThies and G. STRANG, The solution of nonlinear finite element equations, International Journal for Numerical Methods in Engineering, 14 (1979), pp. 1613-1626.

[99] B. Mohammadi and O. Pironneau, Applied Shape Optimization for Fluids, vol. 1, Oxford Science Publications, Oxford, 2001.

[100] V. Mousseau, Implicitly balanced solution of the two-phase flow equations coupled to nonlinear heat conduction, Journal of Computational Physics, 200 (2004), pp. $104-132$.

[101] U. Naumann, The Art of Differentiating Computer Programs, Society for Industrial and Applied Mathematics, Philadephia, 2011.

[102] J. Nocedal, Updating Quasi-Newton Matrices with Limited Storage, Mathematics of Computation, 35 (1980), pp. 773-782.

[103] J. Nocedal ANd M. Overton, Projected Hessian updating algorithms for nonlinearly constrained optimization, SIAM Journal on Numerical Analysis, 22 (1985), pp. 821-850.

[104] J. Nocedal and S. Wright, Numerical Optimization, Springer, New York, 2006. 


\section{BIBLIOGRAPHY}

[105] J. Nordström AND J. Berg, Conjugate heat transfer for the unsteady compressible NavierStokes equations using a multi-block coupling, Computers and Fluids, 72 (2013), pp. 20-29.

[106] Y. NotaY, Aggregation-Based Algebraic Multigrid for Convection-Diffusion Equations, SIAM Journal on Scientific Computing, 34 (2012), pp. A2288-A2316.

[107] C. Paige and M. Saunders, Solution of Sparse Indefinite Systems of Linear Equations, SIAM Journal on Numerical Analysis, 12 (1975), pp. 617-629.

[108] M. Z. PoDowski, Multidimensional modeling of two-phase flow and heat transfer, International Journal of Numerical Methods for Heat \& Fluid Flow, 18 (2008), pp. 491-513.

[109] L. Pontryagin, V. Boltyanskit, R. Gamkrelidze, and E. Mishchenko, The Mathematical Theory of Optimal Processes, Wiley, New York, 1962.

[110] E. Prudencio, R. Byrd, and X. Cai, Parallel Full Space SQP Lagrange-Newton-KrylovSchwarz Algorithms for PDE-Constrained Optimization Problems, SIAM Journal on Scientific Computing, 27 (2006), pp. 1305-1328.

[111] T. Rees, H. Dollar, and A. Wathen, Optimal Solvers for PDE-Constrained Optimization, SIAM Journal on Scientific Computing, 32 (2010), pp. 271-298.

[112] J. W. Ruge and K. Stuben, Algebraic Multigrid (AMG), in Multigrid Methods, Y. Saad and M. H. Schultz, eds., Frontiers in Applied Mathematics, SIAM, Philadelphia, 1987, pp. 73130.

[113] Y. SAAD, Iterative Methods for Sparse Linear Systems, Society for Industrial and Applied Mathematics, Philadelphia, 2nd ed., 2003.

[114] Y. SAAD AND M. H. Schultz, GMRES: a generalized minimal residual algorithm for solving nonsymmetric linear systems, SIAM Journal on Scientific and Statistical Computing, 7 (1986), pp. 856-869.

[115] W. SchmidT, Numerical methods for optimal control problems with ODE or integral equations, in Large-Scale Scientific Computing, I. Lirkov, S. Margenov, and J. Waśniewski, eds., 


\section{BIBLIOGRAPHY}

vol. 3743 of Lecture Notes in Computer Science, Springer Berlin Heidelberg, 2006, pp. 255262.

[116] A. Shenoy, E. Cliff, and M. Heinkenschloss, Thermal-Fluid Control Via Finite-Dimensional Approximation. Paper AIAA-96-1910, in Proceedings of the 31st AIAA Thermophysics Conference June 17-20, 1996, New Orleans, LA, AIAA, 1996, pp. 1-11.

[117] D. Silvester, H. Elman, AND A. Ramage, Incompressible Flow and Iterative Solver Software (IFISS) version 3.2. http://wWw.manchester.ac.uk/ifiss/, May 2012. Accessed: 2013-05-17.

[118] D. Silvester and A. Wathen, Fast Iterative Solution of Stabilised Stokes Systems Part II: Using General Block Preconditioners, SIAM Journal on Numerical Analysis, 31 (1994), pp. 1352-1367.

[119] B. F. Sмith, P. E. BJørstad, and W. Gropp, Domain Decomposition: Parallel Multilevel Methods for Elliptic Partial Differential Equations, Cambridge University Press, Cambridge, 1996.

[120] M. SMooke ANd R. MatTheI, On the solution of nonlinear two-point boundary value problems on successively refined grids, Applied Numerical Mathematics, 1 (1985), pp. 463-487.

[121] H. D. Sterck, U. M. YAng, and J. J. Heys, Reducing complexity in parallel algebraic multigrid preconditioners, SIAM Journal on Matrix Analysis and Applications, 27 (2005), pp. 1019-1039.

[122] M. Stoll and A. Wathen, All-at-once solution of time-dependent Stokes control, Journal of Computational Physics, 232 (2013), pp. 498-515.

[123] A. Toselli and O. Widlund, Domain Decomposition Methods - Algorithms and Theory, vol. 34 of Springer Series in Computational Mathematics, Springer Berlin Heidelberg, 2004.

[124] U. Trottenberg and A. Schuller, Multigrid, Academic Press, Inc., Orlando, 2001. 


\section{BIBLIOGRAPHY}

[125] H. van DER VoRST, Bi-CGSTAB: A fast and smoothly converging variant of Bi-CG for the solution of nonsymmetric linear systems, SIAM Journal on Scientific and Statistical Computing, 13 (1992), pp. 631-644.

[126] A. Wathen and D. Silvester, Fast Iterative Solution of Stabilised Stokes Systems Part I: Using Simple Diagonal Preconditioners, SIAM Journal on Numerical Analysis, 30 (1993), pp. 630-649.

[127] D. C. WILcox, Turbulence Modeling for CFD, DCW Industries Inc., La Canada, CA, 1993.

[128] M. H. WRIGHT, The interior-point revolution in optimization: history, recent developments, and lasting consequences, Bulletin of the American Mathematical Society. New Series, 42 (2005), pp. 39-56.

[129] C.-T. Wu and H. C. Elman, Analysis and comparison of geometric and algebraic multigrid for convection-diffusion equations, SIAM Journal on Scientific Computing, 28 (2006), pp. 2208-2228.

[130] Y. Yan, S. Antal, B. Edge, D. Keyes, D. Shaver, I. Bolotnov, and M. Podowski, Parallel Linear Solvers for Simulations of Reactor Thermal Hydraulics, in 14th International Topical Meeting on Nuclear Reactor Thermal Hydraulics (NURETH-14) Sept. 25-30, 2011, Toronto, Canada, 2011.

[131] Y. Yan And D. E. Keyes, Smooth and Robust Solutions for Dirichlet Boundary Control of Fluid-Solid Conjugate Heat Transfer Problems, Journal of Computational Physics, 281 (2015), pp. 759-786.

[132] H. YAng AND X.-C. CAI, Parallel fully implicit two-grid methods for distributed control of unsteady incompressible flows, International Journal for Numerical Methods in Fluids, 72 (2013), pp. 1-21.

[133] H. Yang, E. E. Prudencio, and X.-C. CAi, Fully implicit Lagrange-Newton-Krylov-Schwarz algorithms for boundary control of unsteady incompressible flows, International Journal for Numerical Methods in Engineering, 91 (2012), pp. 644-665. 


\section{BIBLIOGRAPHY}

[134] H. Yücel, M. Heinkenschloss, and B. Karasözen, Distributed optimal control of diffusionconvection-reaction equations using discontinuous Galerkin methods, in Numerical Mathematics and Advanced Applications 2011, A. Cangiani, R. L. Davidchack, E. Georgoulis, A. N. Gorban, J. Levesley, and M. V. Tretyakov, eds., Springer Berlin Heidelberg, 2013, pp. 389-397. 


\section{Part VI}

\section{Appendices}




\section{Appendix A}

\section{Derivation of the Optimality Conditions}

\section{in Section 4.3}

In this section, we derive the Optimality Conditions for the DVBC of the CHT process (2.1)-(2.4). The heat diffusion in the solid domain $\Omega_{1}$ is described as

$$
-k_{s}\left(\frac{\partial^{2} T_{s}}{\partial x^{2}}+\frac{\partial^{2} T_{s}}{\partial y^{2}}\right)=q,
$$

with boundary conditions (BCs)

$$
\left\{\begin{array}{rlll}
k_{s} \frac{\partial T_{s}}{\partial y}-k_{f} \frac{\partial T_{f}}{\partial y} & =0 & \text { on } \Gamma_{c} \\
T_{s}-T_{f} & =0 & \text { on } \Gamma_{c} \\
\frac{\partial T_{s}}{\partial n} & =0 & \text { on } \Gamma_{1}, \Gamma_{2}, \Gamma_{3},
\end{array}\right.
$$

where $q$ represents the volumetric heat source. We note that an alternative way to write (A.2) is $\mathbf{n} \cdot\left(k_{f} \nabla T_{f}-k_{s} \nabla T_{s}\right)=0$.

For the convenience in the derivation of the optimality conditions, we write the incompressible Navier-Stokes equations in Cartesian coordinates notation, instead of tensor or index notation, 
namely, $\mathbf{u}=(u, v)^{T}$

$$
\begin{aligned}
u \frac{\partial u}{\partial x}+v \frac{\partial u}{\partial y}+\frac{\partial p}{\partial x}-v\left(\frac{\partial^{2} u}{\partial x^{2}}+\frac{\partial^{2} u}{\partial y^{2}}\right) & =0 \\
u \frac{\partial v}{\partial x}+v \frac{\partial v}{\partial y}+\frac{\partial p}{\partial y}-v\left(\frac{\partial^{2} v}{\partial x^{2}}+\frac{\partial^{2} v}{\partial y^{2}}\right) & =0 \\
\frac{\partial u}{\partial x}+\frac{\partial v}{\partial y} & =0
\end{aligned}
$$

with no-slip wall, inlet and outlet BCs as

$$
\left\{\begin{aligned}
\mathbf{u} & =(0,0)^{T} & & \text { on } \Gamma_{c}, \Gamma_{4} \\
\mathbf{u} & =\mathbf{g} & & \text { on } \Gamma_{i} \\
-p \mathbf{n}+v\left(\nabla \mathbf{u}+\nabla \mathbf{u}^{T}\right) \mathbf{n} & =0 & & \text { on } \Gamma_{o},
\end{aligned}\right.
$$

where $\mathbf{g}$ is the inlet velocity boundary control with nonzero components in $x$-direction only.

Assuming that the thermal transport in the fluid-solid system is forced convection and the buoyancy effect can be neglected, we decouple the fluid internal energy equation from the mass and momentum equations and write it as

$$
-k_{f}\left(\frac{\partial^{2} T_{f}}{\partial x^{2}}+\frac{\partial^{2} T_{f}}{\partial y^{2}}\right)+\mathbf{u} \cdot \nabla T_{f}=0,
$$

with adiabatic, inlet and outlet $\mathrm{BCs}$ as

$$
\left\{\begin{aligned}
k_{f} \frac{\partial T_{f}}{\partial y}-k_{s} \frac{\partial T_{s}}{\partial y} & =0 & & \text { on } \Gamma_{c} \\
T_{f}-T_{s} & =0 & & \text { on } \Gamma_{c} \\
T_{f} & =T_{0} & & \text { on } \Gamma_{i} \\
\frac{\partial T_{f}}{\partial n} & =0 & & \text { on } \Gamma_{4}, \Gamma_{o},
\end{aligned}\right.
$$

where $T_{0}$ is inlet temperature boundary condition. As we have shown in (A.5), in the optimization problem, the fluid internal energy equation is coupled with the fluid kinematics, although $T_{f}$ and $\mathbf{u}$ is decoupled in the forward simulation. Without loss of generality, we assume $\beta_{2}=0$ in (2.4) to simplify the notation in this derivation. (However, we emphasize that $\beta_{2}>0$ has to be enforced on both the continuous and the discrete levels to ensure the well-posedness of the problem (2.1)-(2.4). This is actually one of the main results of this study. See Part II for details.) The objective function (2.4) of the optimal control problem is given by

$$
\mathcal{J}\left(\mathbf{u}, p, T_{f}, T_{s}, \mathbf{g}\right):=\frac{1}{2} \int_{\Omega_{d}}\left(T-T_{d}\right)^{2} d x+\frac{\beta}{2} \int_{\Gamma_{i}}|\mathbf{g}|^{2} d s,
$$


where $T_{d}$ is the desired temperature prescribed at the fluid-solid interface and $\Omega_{d}$ denotes the observation domain where the measurement of the fluid temperature is taken. Without loss of generality, we set $\Omega_{d}$ as a subset of the interface $\Gamma_{c}$. The second term in the equation above represents the cost of the control. It regularizes the optimal control problems in $\mathbf{g}$ and limits the control size. Finally, we arrive at a non-convex infinite dimensional optimization problem in functional spaces, due to the nonlinear Navier-Stokes PDE constraints.

There are typically two approaches to prove the existence of optimal controls. The first is to choose the appropriate functional spaces for state equations and inequality constraints, and utilize the solution space structure to construct adjoint equations, gradient variational inequalities, and minimizing sequences in state and control variables towards optimal. This approach needs very careful consideration on the choice of functional spaces and their dual and is of great theoretical interest. The second approach is using Lagrange multiplier theory in function spaces with regularity assumptions on the structure of the function spaces (constraint qualification) [76]. The latter is quite general and can be applied to optimal control problem formally. Here, we follow this second approach. Also, see [62,87]. Introducing Lagrange multipliers, $\theta_{s}, \theta_{f}, \boldsymbol{\xi} \equiv\left(\xi_{u}, \xi_{v}\right)^{T}, \mu$, and $\tau$ for state variables $T_{s}, T_{f}, \mathbf{u} \equiv(u, v)^{T}, p$ and the boundary control $\mathbf{u}=\mathbf{g}$ on $\Gamma_{i}$, respectively, we obtain 
the Lagrangian of the system

$$
\begin{aligned}
\mathcal{L} & \equiv \mathcal{L}\left(T_{s}, T_{f}, u, v, p, \boldsymbol{\sigma}, \theta_{s}, \theta_{f}, \xi_{u}, \xi_{v}, \mu, \boldsymbol{\tau}\right) \\
& =\frac{1}{2} \int_{\Omega_{d}}\left|T-T_{d}\right|^{2} d x d y+\frac{\beta}{2} \int_{\Gamma_{i}}|\mathbf{g}|^{2} d s \\
& -\int_{\Omega_{1}} \theta_{s}\left(-k_{s}\left(\frac{\partial^{2} T_{s}}{\partial x^{2}}+\frac{\partial^{2} T_{s}}{\partial y^{2}}\right)-q\right) d x d y \\
& -\int_{\Omega_{2}} \theta_{f}\left(-k_{f}\left(\frac{\partial^{2} T_{f}}{\partial x^{2}}+\frac{\partial^{2} T_{f}}{\partial y^{2}}\right)+\mathbf{u} \cdot \nabla T_{f}\right) d x d y \\
& -\int_{\Omega_{2}} \xi_{u}\left(u \frac{\partial u}{\partial x}+v \frac{\partial u}{\partial y}+\frac{\partial p}{\partial x}-v\left(\frac{\partial^{2} u}{\partial x^{2}}+\frac{\partial^{2} u}{\partial y^{2}}\right)\right) d x d y \\
& -\int_{\Omega_{2}} \xi_{v}\left(u \frac{\partial v}{\partial x}+v \frac{\partial v}{\partial y}+\frac{\partial p}{\partial y}-v\left(\frac{\partial^{2} v}{\partial x^{2}}+\frac{\partial^{2} v}{\partial y^{2}}\right)\right) d x d y \\
& -\int_{\Omega_{2}} \mu\left(\frac{\partial u}{\partial x}+\frac{\partial v}{\partial y}\right) d x d y \\
& -\int_{\Gamma_{i}} \xi \cdot \boldsymbol{\sigma} d s \\
& -\int_{\Gamma_{i}}^{\tau} \boldsymbol{\tau} \cdot(\mathbf{u}-\mathbf{g}) d s .
\end{aligned}
$$

By the existence theorem of Lagrange multipliers for the optimal control problem (A.1)-(A.7), we can derive the first order optimality conditions or the Karush-Kuhn-Tucker (KKT) conditions for the orginal control problem via taking the Fréchet derivitives of $\mathcal{L}$ with respect to $T_{s}, T_{f}, u, v$ and $p$ along variations $\delta T_{s}, \delta T_{f}, \delta u, \delta v$ and $\delta p$ respectively and making them zero. We note that all variations need to satisfy the boundary condtions that are not enforced by Lagrange multipliers. For the solid and fluid adjoint internal energy equations, by arbitrarity of the variation $\delta T_{s}$ and $\delta T_{f}$, we have

$$
\begin{aligned}
-k_{s} \Delta \theta_{s} & =0 \quad \text { in } \Omega_{1} \\
-k_{f} \Delta \theta_{f}-\mathbf{u} \cdot \nabla \theta_{f} & =0 \quad \text { in } \Omega_{2},
\end{aligned}
$$


with BCs,

$$
\left\{\begin{array}{rlll}
k_{f} \frac{\partial \theta_{f}}{\partial y}-k_{s} \frac{\partial \theta_{s}}{\partial y}-\chi_{\Omega_{d}}\left(T_{f}-T_{d}\right) & =0 \text { on } \Gamma_{c} \\
\theta_{f}-\theta_{s} & =0 \text { on } \Gamma_{c} \\
\frac{\partial \theta_{s}}{\partial n} & =0 \text { on } \Gamma_{1}, \Gamma_{2}, \Gamma_{3} \\
\frac{\partial \theta_{f}}{\partial n} & =0 & \text { on } \Gamma_{4} \\
\theta_{f} & =0 & \text { on } \Gamma_{i} \\
k_{f} \frac{\partial \theta_{f}}{\partial x}+u \theta_{f} & =0 & \text { on } \Gamma_{o} .
\end{array}\right.
$$

For the fluid adjoint mass and momentum equations in $\Omega_{2}$, we have

$$
\begin{aligned}
\xi_{u} \frac{\partial u}{\partial x}+\xi_{v} \frac{\partial v}{\partial x}-u \frac{\partial \xi_{u}}{\partial x}-v \frac{\partial \xi_{u}}{\partial y}-\frac{\partial \mu}{\partial x}-v\left(\frac{\partial^{2} \xi_{u}}{\partial x^{2}}+\frac{\partial^{2} \xi_{u}}{\partial y^{2}}\right)+\theta_{f} \frac{\partial T_{f}}{\partial x}= & 0 \\
\xi_{u} \frac{\partial u}{\partial y}+\xi_{v} \frac{\partial v}{\partial y}-u \frac{\partial \xi_{v}}{\partial x}-v \frac{\partial \xi_{v}}{\partial y}-\frac{\partial \mu}{\partial y}-v\left(\frac{\partial^{2} \xi_{v}}{\partial x^{2}}+\frac{\partial^{2} \xi_{v}}{\partial y^{2}}\right)+\theta_{f} \frac{\partial T_{f}}{\partial y}= & 0 \\
\frac{\partial \xi_{u}}{\partial x}+\frac{\partial \xi_{v}}{\partial y}= & 0,
\end{aligned}
$$

with BCs,

$$
\left\{\begin{array}{rlll}
\xi_{u} & =0 & \text { on } \Gamma_{i}, \Gamma_{c}, \Gamma_{4} \\
\xi_{v} & =0 & \text { on } \Gamma_{i}, \Gamma_{c}, \Gamma_{4} \\
-\mu \mathbf{n}+v\left(\nabla \boldsymbol{\xi}+\nabla \boldsymbol{\xi}^{T}\right) \mathbf{n}+(\mathbf{u} \cdot \mathbf{n}) \xi & = & \text { on } \Gamma_{o} .
\end{array}\right.
$$

The Lagrange multiplier $\tau$, is reduced to the weakly-posed boundary inflow stress of the adjoint Navier-Stokes equations, namely,

$$
-\mu \mathbf{n}+\nu\left(\nabla \boldsymbol{\xi}+\nabla \boldsymbol{\xi}^{T}\right) \mathbf{n}-\boldsymbol{\tau}=0 \quad \text { on } \quad \Gamma_{i} .
$$

Next, we derive the decision equation. Taking the Fréchet derivitives of $\mathcal{L}$ with respect to $\mathbf{g}$ along vartions $\delta \mathbf{g}$, we obtain the reduced gradient equations of the optimality system

$$
\tau+\beta \mathbf{g}=0 \quad \text { on } \quad \Gamma_{i}
$$




\section{Appendix B}

\section{Computer Codes for Multiphysics}

\section{Controls}

\section{B.1 System and Software Requirements}

The code has been tested on a Windows 7 system that runs Matlab version 7.6.0.324 (R2008a). The information provided here is in the hope that it will be useful, but WITHOUT ANY WARRANTY, and without even the implied warranty of MERCHANTABILITY or FITNESS FOR A PARTICULAR PURPOSE. The code is archived at the link: http://goo.gl/MbS7D9.

This code is to demonstrate an numerical scheme with smoothed algorithms and numerical continuations for generating smooth and robust optimal controls for the conjugate heat transfer process. It is an independent set of codes that makes function calls to the library IFISS, Incompressible Flow $\&$ Iterative Solver Software $[47,117]$. Therefore, before any code run, one may download IFISS 3.2 from the link: http://www.cs.umd.edu/ elman/ifiss/download.html, and follow the instruction and IFISS documentation listed there. Please read the License Information for IFISS for its relevant usage, and the document of IFISS for citation.

The input parameters used in the optimal control models are passed through interactive command line. A snapshot of the input for the Numerical Test Case 1 in Section 7.1 is as follows:

* * *optimization of CHT problem demo* * * 
flow in a CHT setting $[\theta, \mathrm{L}] \times[\theta, \mathrm{H}]$

$* * * * * * *$ Grid generation $* * * * * *$.

***fluid velocity and pressure*****.

$* * * * * * * * * * * * * * * * * * * *$.

Input the velocity domain height Hv (default 1): 0.75

Input the domain width L (default 1) :

Input the number of elements in $\mathrm{x}$ direction (default 2): 16

Input the multiplication factor in y-direction (default Hv/L) :

Number of elements in y-direction is: 12

**Grid generation for fluid velocity and pressure**.

Grid statistics Number of elements: 192 ( $16 \times 12$ )

$\mathrm{hxmin}=0.0625 \mathrm{hxmax}=0.0625$

hymin $=0.0625$ hymax $=0.0625$

Grid partitioning done in 0.035 seconds

Velocity and Pressure Grid data saved in box_grid1h_v.mat.

$* * * * * * *$ Grid generation $* * * * * *$.

$* * * * *$ fluid/solid temperature $* * * *$.

$* * * * * * * * * * * * * * * * * * * * * * *$.

The temperature domain length is: 1

Input the temperature domain height Ht (default ==L) :

Number of elements in y-direction is: 16

Input the multiplication factor in y-direction (default Ht/L) :

Number of elements in y-direction is: 16

**Grid generation for fluid velocity and pressure**.

Grid statistics Number of elements: 256 ( 16 x 16)

$\mathrm{hxmin}=0.0625 \mathrm{hxmax}=0.0625$

hymin $=0.0625$ hymax $=0.0625$

Grid partitioning done in 0.030 seconds

Temperature Grid data saved in box_grid1h_t.mat.

SPACE DISCRETIZATION OF THE CHT PROBLEM ( $\mathrm{u}, \mathrm{p}, \mathrm{T})$ :

Setting up Q2-Q1-Q2 matrices:

setting up Q2-Q1 matrices... done

Input the fluid domain heat coefficient (default 2) : 0.1

Input the fluid domain heating source (default $\theta$ ) :

Input the solid domain heat coefficient (default 1) :

Input the solid domain heating source (default 6) :

Number of velocity elements: 192

Number of temperature elements: 256

Number of velocity dofs $(x+y): 1650$ 
Number of pressure dofs: 221

Number of temperature dofs: 1089

Total number of dofs: 2960

Temperature System setup in 0.057 seconds

done

setting up Q2 diffusion matrices... done

Grid data saved in $* * *$ box_grid1h_v.mat and box_grid1h_t.mat ***

System matrices saved in $* * *$ cht_nobc.mat $* * *$

Generate all the parameters for the nonlinear CHT problem:

fluid k-viscosity (default 1.0) : 0.001

Objective function penalty parameter: beta1 for L2 control term (default 1.0) :

Objective function penalty parameter: beta2 for $\mathrm{H} 1$ control term (default 1.Q) :

nonlinear tolerance (default 1.e-8) :

uniform/exponential streamlines $1 / 2$ (default uniform) :

\section{B.2 The Main Routine}

To describe the hierarchy of the optimal control code, we list the commented main routine in the following.

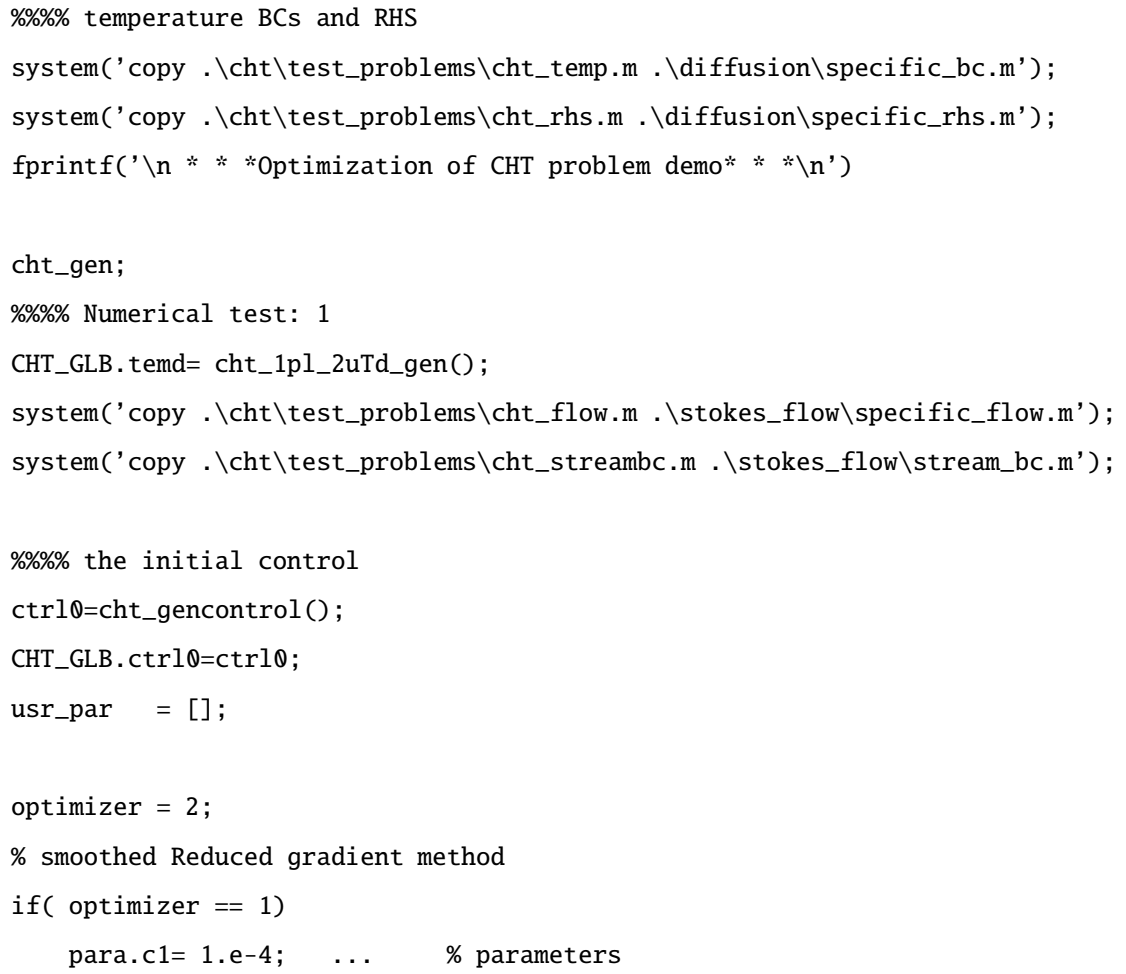


[ ctrl, iter,iterls, iflag ] = cht_reducedgradient_wp ( $\operatorname{ctrl} 0$, para);

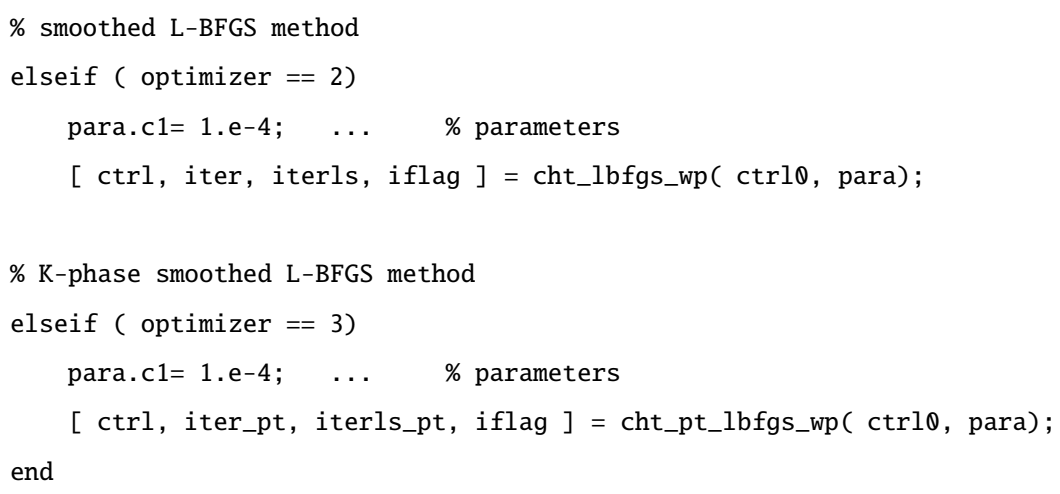

The partitioning of the physical domain and the assembly of initial FEM matrices are carried out in cht_gen, the target temperature profile is generated in cht_1pl_2uTd_gen, and the initial control is generated in cht_gencontrol. We have adopted the Modular Programming paradigm to enhance the readability of the code, and the optimization framework is based on [72]. 


\section{Appendix C}

\section{The Lagrange-Newton-Reduced-Krylov (LNRK) Framework for Multiphysics Controls}

\section{C.1 Overview}

Many nonlinear optimization problems, either constrained or unconstrained, are solved by using solution sequencing techniques, in which solutions of a sequence of subproblems are forced to converge to the global minimum. Among those techniques, the sequential quadratic programming (SQP) method $[23,27,53,104]$ and the interior point method $[78,104,128]$ are the most efficient ones. Both methods have been applied successfully to optimal control with PDE constraints. See $[42,57,78]$. For the optimal control problem of CHT processes, we have chosen the SQP method, which is derived from the Lagrange-Newton framework. In this method, there are two approaches to solving the quadratic programming subproblems, namely the full space SQP method, in which the state and the control variables are solved and updated simultaneously, and the reduced space SQP method, in which the linearized Karush-Kuhn-Tucker (KKT) system is reduced to a much smaller dense linear system in the control space only.

In multiphysics boundary control problems where the actuation device is only effective in a 
single physics and only on a very small part of the domain, such as in optimal control of CHT processes, the discrete control space has a much smaller dimension than the discrete state space. This implies that the reduced SQP method is a more suitable choice. In fact, the reasons are threefold. First, each of the state, the adjoint, and the reduced Hessian systems in the reduced SQP method has a much smaller size than the linearized KKT system in the full space. Therefore, the solution process of the reduced SQP method consumes much less memory. Moreover, the separation between the state and the adjoint systems in the reduced SQP method has made it convenient to reuse the solvers and preconditioners of the state and adjoint PDEs. This is a quite desirable feature for complex multiphysics simulations. Furthermore, the reduced Hessian in the reduced SQP method is dense, but much smaller and much better conditioned than the full discrete KKT matrix in the full space SQP method.

In Section C.2, we derive the Lagrange-Newton reduced space approach. The quasi-Newton approximation for the reduced Hessian is discussed in Section C.3. In Section C.4, we make connections between the reduced space approach and the implicitly constrained optimization problem. The merit function for the line search process is studied in Section C.5, and the inexactness issue is discussed in Section C.6.

\section{C.2 Lagrange-Newton Reduced Space Approach}

In constrained optimization problems, the optimality conditions are characterized by the stationarity of the Lagrangian function [29], namely the Karush-Kuhn-Tucker (KKT) conditions. The LagrangeNewton (or SQP) method applies Newton linearization to the KKT conditions and solves for the optimal solution iteratively. It can be shown that under the assumptions of constraint qualifications, the Lagrange-Newton method defines a SQP algorithm, and the Newton linearization of the KKT conditions provides a quadratic approximation for both the PDE constraints and the Lagrangian function [4]. 
We start with the constrained optimization problem

$$
\left\{\begin{array}{cl}
\underset{(\mathbf{y}, \mathbf{u})}{\operatorname{minimize}} & f(\mathbf{y}, \mathbf{u}) \\
\text { subject to } & \mathbf{h}(\mathbf{y}, \mathbf{u})=0
\end{array}\right.
$$

where $\mathbf{u}$ is the control variable, $\mathbf{y}$ the state variable, $f(\mathbf{y}, \mathbf{u})$ the objective function, and $\mathbf{h}(\mathbf{y}, \mathbf{u})=0$ the constraints, which is usually a system of ODEs [115] or PDEs [21,76]. When the Jacobian of the $\mathbf{h}(\mathbf{y}, \mathbf{u})$ is nonsingular, the constraints $\mathbf{h}(\mathbf{y}, \mathbf{u})=0$ define an implicit function $\mathbf{y}:=\mathbf{y}(\mathbf{u})$. In practice, there are various interpretations of the control variable $\mathbf{u}$, for instance, $\mathbf{u}$ is the reconstruction parameter $[10,68]$ in inverse problems, and $\mathbf{u}$ is the shape parameter [78] in shape optimization. To simplify the notation, we introduce a new variable $\mathbf{x}:=(\mathbf{y}(\mathbf{u}), \mathbf{u})$. The optimization problem (C.1) then becomes

$$
\begin{cases}\underset{\mathbf{x}}{\operatorname{minimize}} & f(\mathbf{x}) \\ \text { subject to } & \mathbf{h}(\mathbf{x})=0 .\end{cases}
$$

Moreover, we introduce the Lagrangian function

$$
\mathcal{L}(\mathbf{x}, \lambda):=f(\mathbf{x})+\lambda^{T} \mathbf{h}(\mathbf{x}) .
$$

By taking variations of $\mathcal{L}$ with respect to the primal variable $\mathbf{x}$ and the multiplier $\lambda$ respectively and requiring their stationarity at local extrema, we obtain a nonlinear system of the first order optimality conditions for (C.2)

$$
\left\{\begin{array}{l}
\mathcal{L}_{\mathbf{x}}(\mathbf{x}, \lambda)=\mathbf{g}(\mathbf{x})+\mathbf{C}(\mathbf{x})^{T} \lambda=0 \\
\mathcal{L}_{\lambda}(\mathbf{x}, \lambda)=\mathbf{h}(\mathbf{x})=0
\end{array}\right.
$$

where $\mathbf{g}(\mathbf{x})$ is the gradient of the function $\mathbf{f}(\mathbf{x})$, and the $i$-th row of the matrix $\mathbf{C}(\mathbf{x})$ is the gradient of the $i$-th component of the constraints $\mathbf{h}(\mathbf{x})$. We denote by $\mathbf{H}(\mathbf{x})$ the Hessian of the Lagrangian $\mathcal{L}(\mathbf{x}, \lambda)$ and apply Newton's method to the KKT conditions (C.4). Then, we obtain the Newton system for the primal variable $\mathbf{x}$ and multiplier $\lambda$

$$
\left(\begin{array}{cc}
\mathbf{H}(\mathbf{x}) & \mathbf{C}(\mathbf{x})^{T} \\
\mathbf{C}(\mathbf{x}) & 0
\end{array}\right)\left(\begin{array}{l}
\mathbf{d}_{\mathbf{x}} \\
\mathbf{d}_{\lambda}
\end{array}\right)=-\left(\begin{array}{c}
\mathbf{g}(\mathbf{x})+\mathbf{C}(\mathbf{x})^{T} \lambda \\
\mathbf{h}(\mathbf{x})
\end{array}\right)=-\left(\begin{array}{c}
\mathcal{L}_{\mathbf{x}}(\mathbf{x}, \lambda) \\
\mathbf{h}(\mathbf{x})
\end{array}\right)
$$


or equivalently,

$$
\left(\begin{array}{cc}
\mathbf{H}(\mathbf{x}) & \mathbf{C}(\mathbf{x})^{T} \\
\mathbf{C}(\mathbf{x}) & 0
\end{array}\right)\left(\begin{array}{c}
\mathbf{d}_{\mathbf{x}} \\
\lambda
\end{array}\right)=-\left(\begin{array}{c}
\mathbf{g}(\mathbf{x}) \\
\mathbf{h}(\mathbf{x})
\end{array}\right) .
$$

We have omitted the iteration numbers $k$ and $k+1$ and the notation $\left(\mathbf{x}^{k+1}, \lambda^{k+1}\right)^{T}=\left(\mathbf{x}^{k}, \lambda^{k}\right)^{T}+$ $\left(\mathbf{d}_{\mathbf{x}}, \mathbf{d}_{\lambda}\right)^{T}$, and in what follows, we omit the dependency on $\mathbf{x}$ in (C.5) and (C.6). It can be shown that the system (C.6) defines a quadratic program [4]

$$
\left\{\begin{array}{cl}
\underset{\mathbf{d}_{\mathbf{x}}}{\operatorname{minimize}} & \frac{1}{2} \mathbf{d}_{\mathbf{x}}^{T} \mathbf{H} \mathbf{d}_{\mathbf{x}}+\mathbf{g}^{T} \mathbf{d}_{\mathbf{x}} \\
\text { subject to } & \mathbf{C} \mathbf{d}_{\mathbf{x}}+\mathbf{h}=0 .
\end{array}\right.
$$

Therefore, the Lagrange-Newton method is also referred to as the SQP method. In general, two approaches exist for solving the system (C.5), namely the full space approach and the reduced space approach. In the full space SQP method, the Newton system (C.5) is solved in the primal and the dual spaces simultaneously $[3,110,132,133]$; in the reduced space SQP method $[22,57,103]$, (C.5) is reduced to a much smaller system in a subspace of the primal variable $\mathbf{x}=(\mathbf{y}(\mathbf{u}), \mathbf{u})$, and the subspace is almost always chosen to be the control space $\mathbf{u}$ in optimal control problems with PDEs constraints $[3,28,57,76]$.

For deriving the reduced space SQP method, we use (C.6) and introduce the subspace decomposition for the Newton update $\mathbf{d}_{\mathbf{x}}$ of the primal variable $\mathbf{x}$

$$
\mathbf{d}_{\mathbf{x}}:=\mathbf{Y} \mathbf{d}_{\mathbf{y}}+\mathbf{U} \mathbf{d}_{\mathbf{u}}
$$

We assume that the columns of the matrix $\mathbf{Y}$ are the basis of the range space of $\mathbf{C}^{T}$ and the columns of $\mathbf{U}$ are the basis of the null space of $\mathbf{C}$, namely $\mathbf{C} \mathbf{U}=0$. From the expansion of the second row in (C.6), we obtain the state equation

$$
\text { (State) } \quad \mathbf{C Y d} \mathbf{d}_{\mathbf{y}}=-\mathbf{h} \text { or } \quad \mathbf{C d}_{\mathbf{x}}=-\mathbf{h} \text {. }
$$

By multiplying the first row of (C.6) by $\mathbf{Y}^{T}$ from left, we obtain the adjoint equation

$$
\text { (Adjoint) } \quad \mathbf{Y}^{T} \mathbf{C}^{T} \lambda=-\mathbf{Y}^{T}\left(\mathbf{H d}_{\mathbf{x}}+\mathbf{g}\right) \approx-\mathbf{Y}^{T} \mathbf{g},
$$


where in the last step we have used the fact that $\mathbf{d}_{\mathbf{x}}$ eventually converges to zero in the nonlinear optimization iterations for a fixed $\mathbf{g}$ [104]. Finally, by multiplying the first row of (C.5) by $\mathbf{U}^{T}$ from left, we obtain the reduced Hessian equation for the control update $\mathbf{d}_{\mathbf{u}}$

$$
\begin{aligned}
\mathbf{U}^{T} \mathbf{H} \mathbf{d}_{\mathbf{x}}+\mathbf{U}^{T} \mathbf{C}^{T} \mathbf{d}_{\lambda} & =-\mathbf{U}^{T} \mathcal{L}_{\mathbf{x}}, \text { where }-\mathbf{U}^{T} \mathcal{L}_{\mathbf{x}}=-\mathbf{U}^{T} \mathbf{g} \\
\text { (Reduced) } \quad \mathbf{U}^{T} \mathbf{H Y} \mathbf{Y} \mathbf{d}_{\mathbf{y}}+\mathbf{U}^{T} \mathbf{H} \mathbf{U} \mathbf{d}_{\mathbf{u}} & =-\mathbf{U}^{T} \mathcal{L}_{\mathbf{x}} \\
\mathbf{U}^{T} \mathbf{H} \mathbf{U} \mathbf{d}_{\mathbf{u}} & \approx-\mathbf{U}^{T} \mathcal{L}_{\mathbf{x}},
\end{aligned}
$$

where we have used $\mathbf{U}^{T} \mathbf{C}^{T}=0$ and the fact that $\mathbf{d}_{\mathbf{y}}$ eventually converges to zero in the nonlinear optimization iterations for a fixed $\mathbf{d}_{\mathbf{u}}$ [104]. In practice, the reduced SQP problem (C.11) can be solved by two different methods, namely the Newton reduced SQP method where the matrix-vector multiplication of the reduced Hessian $\mathbf{U}^{T} \mathbf{H U}$ is carried out exactly, and the quasi-Newton reduced SQP method where the matrix-vector multiplication of $\left(\mathbf{U}^{T} \mathbf{H U}\right)^{-1}$ is computed by quasi-Newton approximations, such as the L-BFGS method $[98,102]$.

\section{C.3 Quasi-Newton Approximation for the Reduced Hessian}

For optimization problems with PDE systems as constraints, it is not rare that the second order information of the objective function is too complex or too expensive to obtain. However, the first order information is always accessible $[28,62,76]$. Therefore, it is practical in complex problems, such as CHT controls, to seek quasi-Newton approximations for the reduce Hessian $\mathbf{U}^{T} \mathbf{H U}$ or its inverse. The advantages of using quasi-Newton methods for (C.11) are threefold. First, quasi-Newton methods are gradient-based methods, which do not require the Hessian of the Lagrangian function. Second, these methods are memory and computationally efficient. They do not store any part of the Hessian or solve for exact Hessian-vector multiplications, which are required by Newton's method, and which involve the solutions of auxiliary state and adjoint systems that are computationally very expensive. In fact, the quasi-Newton method only solves a much smaller linear system with a dense symmetric positive definite (SPD) reduced Hessian [57] and store a limited amount of vectors to recover the approximated Hessian-vector multiplications. Third, these methods has a local superlinear convergence rate. The quasi-Newton methods use successive gradient information obtained 
from previous iterations to build up a local approximation to the reduced Hessian or its inverse. It can be shown that these methods have a superlinear convergence rate when guarded with an efficient line search procedure [104]. Furthermore, in quasi-Newton approximations, we can easily incorporate the numerical smoothing device into the two-loop recursion, given that numerical smoothing is required by CHT control problems [131]. Also, see Section 6.4 for a detailed discussion.

For optimal control of PDE systems, it is important to take account of the partitioning between the state variable $\mathbf{y}$ and the control variable $\mathbf{u}$. In fact, this partitioning has been implicitly taken care of and been available in almost all existing software for PDE simulations, due to that the control device can be either boundary conditions $[3,26]$, source terms [122], shape parameters [78], or unknown coefficients $[10,67,68]$. Therefore, once the range-null space decomposition of the state Jacobian $\mathbf{C}$ in (C.8) is aligned with the state-control space partitioning, reusing the existing PDE solvers and preconditioners is possible for the optimal control problems.

We recall the structure of the primal variable $\mathbf{x}:=(\mathbf{y}(\mathbf{u}), \mathbf{u})$, and partition the Jacobian matrix as $\mathbf{C}:=\left[\mathbf{C}_{\mathbf{y}} \mid \mathbf{C}_{\mathbf{u}}\right]$. In practice, the range-null space decomposition using

$$
\mathbf{U}=\left(\begin{array}{c}
-\mathbf{C}_{\mathbf{y}}^{-1} \mathbf{C}_{\mathbf{u}} \\
\mathbf{I}
\end{array}\right) \quad \text { and } \quad \mathbf{Y}=\left(\begin{array}{l}
\mathbf{I} \\
0
\end{array}\right)
$$

has been applied successfully in many optimal control problems $[28,57,69]$. We summarize the L-BFGS reduced SQP method in Algorithm C.3.1.

\section{C.4 Connections to the Implicitly Constrained Optimization Problem}

Our derivation of the reduced SQP method is on the discrete level, namely in finite dimensional spaces where the Jacobian of the state equation is defined and partitioned. In fact, there is a continuous analogue of the reduced SQP method in infinite dimensional function spaces. See $[28,72,76]$. We consider again the constrained optimization problem (C.1) with the state variable being an implicit function, namely $\mathbf{y}:=\mathbf{y}(\mathbf{u})$ defined by $\mathbf{h}(\mathbf{y}, \mathbf{u})=0$. We then arrive at the implicitly constrained [72] or the reduced $[28,76]$ optimization problem

$$
\{\underset{\mathbf{u}}{\operatorname{minimize}} \hat{f}(\mathbf{u}):=f(\mathbf{y}(\mathbf{u}), \mathbf{u})
$$




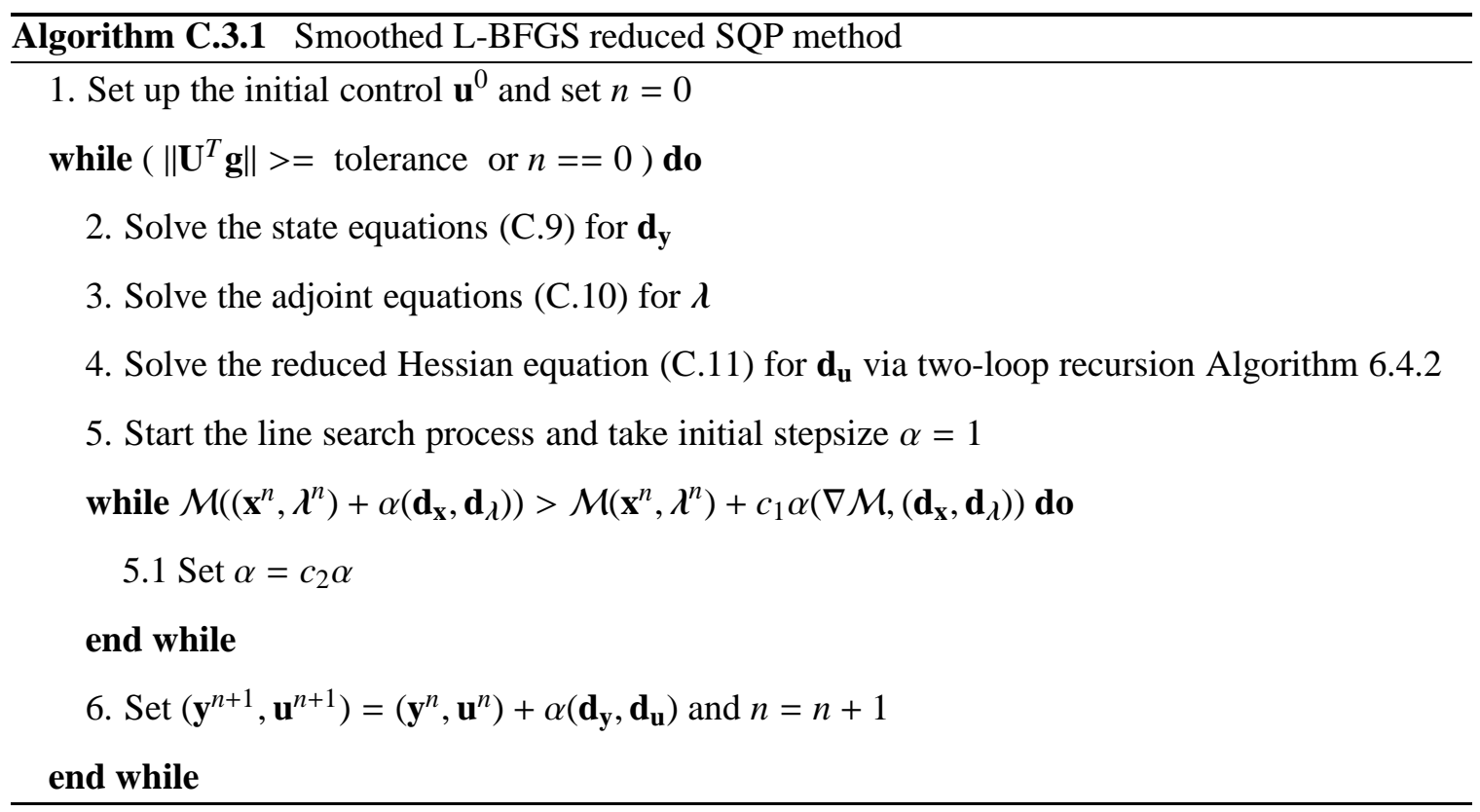

For the optimization problem (C.13), many gradient-based methods are applicable, such as the Steepest Descent method, the nonlinear conjugate gradient (CG) methods, and the quasi-Newton method. Their convergence rates range from linear to superlinear [28, 104]. See [131] or Chapter 6-7 for the application of the Steepest Descent method and the quasi-Newton method to optimal control of CHT processes. When the reduced Hessian $\nabla^{2} \hat{f}(\mathbf{u})$ is accessible, the Newton CG method can be employed to achieve the local quadratic convergence by solving Newton's equation of the unconstrained optimization problem (C.13)

$$
\nabla^{2} \hat{f}(\mathbf{u}) \mathbf{d}_{\mathbf{u}}=-\nabla \hat{f}(\mathbf{u}),
$$

where $\mathbf{d}_{\mathbf{u}}$ is the Newton direction of the control variable $\mathbf{u}$. There are generally two approaches to computing the reduced gradient $\nabla \hat{f}(\mathbf{u})$, namely the sensitivity approach and the adjoint approach $[62,76]$. To see the connections between the reduced SQP method in Section C.2 and the Newton CG method for the implicitly constrained optimization problem, we take the adjoint approach. Again, we denote by $\mathbf{C}$ the Jacobian of the state equation $\mathbf{h}(\mathbf{y}, \mathbf{u})=0$, and we partition the state $\mathbf{y}$ and the control $\mathbf{u}$ as before, namely $\mathbf{C}:=\left[\mathbf{C}_{\mathbf{y}} \mid \mathbf{C}_{\mathbf{u}}\right]$. After applying the implicit function theorem $[72,76]$ on 
$\mathbf{h}(\mathbf{y}, \mathbf{u})=0$, we have

$$
\begin{aligned}
\mathbf{C}_{\mathbf{y}} \mathbf{y}_{\mathbf{u}} & =-\mathbf{C}_{\mathbf{u}} \\
\mathbf{y}_{\mathbf{u}} & =-\mathbf{C}_{\mathbf{y}}^{-1} \mathbf{C}_{\mathbf{u}} .
\end{aligned}
$$

Therefore, the reduced gradient is

$$
\begin{aligned}
\nabla \hat{f}(\mathbf{u}) & =\mathbf{y}_{\mathbf{u}}^{T} \nabla_{\mathbf{y}} f(\mathbf{y}(\mathbf{u}), \mathbf{u})+\nabla_{\mathbf{u}} f(\mathbf{y}(\mathbf{u}), \mathbf{u}) \\
& =-\mathbf{C}_{\mathbf{u}}^{T} \mathbf{C}_{\mathbf{y}}^{-T} \nabla_{\mathbf{y}} f(\mathbf{y}(\mathbf{u}), \mathbf{u})+\nabla_{\mathbf{u}} f(\mathbf{y}(\mathbf{u}), \mathbf{u}) \\
& =\mathbf{U}^{T} \nabla_{\mathbf{x}} f(\mathbf{y}(\mathbf{u}), \mathbf{u}) \\
& =\mathbf{U}^{T} \mathbf{g},
\end{aligned}
$$

where we have denoted the primal variable by $\mathbf{x}:=(\mathbf{y}(\mathbf{u}), \mathbf{u})$, the gradient $\nabla_{\mathbf{x}} f$ by $\mathbf{g}$, and the matrix U by

$$
\mathbf{U}=\left(\begin{array}{c}
\mathbf{C}_{\mathbf{y}}^{-1} \mathbf{C}_{\mathbf{u}} \\
\mathbf{I}
\end{array}\right) .
$$

If we define the multiplier

$$
\text { (Adjoint) } \quad \lambda:=\mathbf{C}_{\mathbf{y}}^{-T} \nabla_{\mathbf{y}} f(\mathbf{y}(\mathbf{u}), \mathbf{u})
$$

the reduced gradient can be written

$$
(\text { Gradient }) \quad \nabla \hat{f}(\mathbf{u})=-\mathbf{C}_{\mathbf{u}}^{T} \lambda+\nabla_{\mathbf{u}} f(\mathbf{y}(\mathbf{u}), \mathbf{u})=\mathcal{L}_{\mathbf{u}} .
$$

For the derivation of the Hessian $\nabla^{2} \hat{f}$ of the reduced objective function $\hat{f}$, we refer to $[72,76]$ and write

$$
\begin{aligned}
\nabla^{2} \hat{f} & =\mathbf{C}_{\mathbf{u}}^{T} \mathbf{C}_{\mathbf{y}}^{-T} \mathcal{L}_{\mathbf{y y}} \mathbf{C}_{\mathbf{y}}^{-1} \mathbf{C}_{\mathbf{u}}+\mathbf{C}_{\mathbf{u}}^{T} \mathbf{C}_{\mathbf{y}}^{-T} \mathcal{L}_{\mathbf{y u}}+\mathcal{L}_{\mathbf{u y}} \mathbf{C}_{\mathbf{y}}^{-1} \mathbf{C}_{\mathbf{u}}+\mathcal{L}_{\mathbf{u u}} \\
& =\mathbf{U}^{T}\left(\begin{array}{cc}
\mathcal{L}_{\mathbf{y y}} & \mathcal{L}_{\mathbf{y u}} \\
\mathcal{L}_{\mathbf{u y}} & \mathcal{L}_{\mathbf{u u}}
\end{array}\right) \mathbf{U} \\
& =\mathbf{U}^{T} \mathbf{H} \mathbf{U}
\end{aligned}
$$

It is evident from (C.16) and (C.20) that the Newton equation (C.14) has the same expression with the reduced Hessian equation (C.11) of the reduced SQP method in Section C.2. However, the reduced SQP method equations (C.9)-(C.11) are different from those of the implicitly constrained 
optimization problem (C.13)-(C.14). At each nonlinear optimization iteration, in reduced SQP method, we are solving one linearized state equation (C.9) for a fixed control $\mathbf{u}$, but in the reduced optimization problem, because $\mathbf{y}:=\mathbf{y}(\mathbf{u})$ is considered as an implicit function, we need to solve the nonlinear state equation $\mathbf{h}(\mathbf{y}, \mathbf{u})=0$ for $\mathbf{y}$ exactly. Moreover, the reduced SQP method is much more efficient, because when the optimization iterate is outside the basins of attraction in Newton or quasi-Newton methods, feasibility of the state equation can be relaxed to avoid oversolving.

\section{C.5 Merit Functions for the Line Search Process}

It is evident from (C.5) that in the SQP method, the feasibility of the state variable $\mathbf{y}$ has been relaxed, namely the solution of (C.5) merely satisfies the linearized constraints $\mathbf{C}(\mathbf{x}) \mathbf{d}_{\mathbf{x}}=-\mathbf{h}(\mathbf{x})$. In other words, the nonlinear constraints $\mathbf{h}(\mathbf{x})=0$ may not be satisfied during the optimization process. Therefore, we need measure both the value of the objective function and the violation of the nonlinear constraints in globalization procedures, such as line search or trust region methods, to determine proper stepsizes. For SQP methods, including both the full and the reduced space approaches, two popular merit functions are the $l_{1}$ merit function [26, 104]

$$
\mathcal{M}_{1}:=\mathbf{f}(\mathbf{x})+\mu_{1}\|\mathbf{h}(\mathbf{x})\|_{1}
$$

and the augmented Lagrangian function $[26,41,69,104,110]$

$$
\begin{aligned}
\mathcal{M}_{2} & :=\mathbf{f}(\mathbf{x})+\lambda^{T} \mathbf{h}(\mathbf{x})+\frac{\mu_{2}}{2}\|\mathbf{h}(\mathbf{x})\|_{2}^{2} \\
& :=\mathcal{L}(\mathbf{x}, \lambda)+\frac{\mu_{2}}{2}\|\mathbf{h}(\mathbf{x})\|_{2}^{2} .
\end{aligned}
$$

It is clear that when the parameters $\mu_{1}$ and $\mu_{2}$ which penalize the violation of the constraints $\mathbf{h}(\mathbf{x})=0$ are small, the optimization step chosen will be mostly reducing the objective function value, and when $\mu_{1}$ and $\mu_{2}$ are large, the step chosen will be mainly reducing the violation of the constraints. Therefore, it is intuitive to choose small parameters at the beginning of the optimization process, since the control is still far from optimal at this stage and the merit function is designed to accept rough estimates of the state variables, which are computationally inexpensive to obtain. Gradually, we increase those parameters and enforce the feasibility when the control is approaching the optimum. 
The drawback of using the $l_{1}$ merit function is the Maratos effect [26, 104], which represents the case that a good line search step is rejected and the superlinear convergence rate is hindered. To overcome this difficulty, two strategies are used in practice. First, a second order correction term can be added to the search direction $\mathbf{d}_{\mathbf{x}}:=\left(\mathbf{d}_{\mathbf{y}}, \mathbf{d}_{\mathbf{u}}\right)$ if the current line search step fails. Second, a nonmonotone line search process can be used. See [104] for more details. The directional derivative for $\mathcal{M}_{1}$ is

$$
\begin{aligned}
\left(\nabla \mathcal{M}_{1}, \mathbf{d}_{\mathbf{x}}\right) & =\left(\nabla \mathbf{f}(\mathbf{x}), \mathbf{d}_{\mathbf{x}}\right)-\mu_{1}\|\mathbf{h}(\mathbf{x})\|_{1} \\
& =\nabla \mathbf{f}^{T}(\mathbf{x}) \mathbf{d}_{\mathbf{x}}-\mu_{1}\|\mathbf{h}(\mathbf{x})\|_{1},
\end{aligned}
$$

and the strategy for choosing the penalty parameters $\mu_{1}$ includes

$$
\mu_{1}>\|\lambda\|_{\infty}
$$

which uses the multiplier $\lambda$ and ensures the descent condition for the $l_{1}$ merit function $\mathcal{M}_{1}$ and $\mathbf{d}_{\mathbf{x}}$.

The augmented Lagrangian function does not suffer from the Maratos effect. But the eveluations of the merit function $\mathcal{M}_{2}$ and its gradient $\nabla \mathcal{M}_{2}$ are more involved. For the search direction $\left(\mathbf{d}_{\mathbf{x}}, \mathbf{d}_{\lambda}\right)$, the directional derivation of $\mathcal{M}_{2}$ is

$$
\begin{aligned}
\left(\nabla \mathcal{M}_{2},\left(\mathbf{d}_{\mathbf{x}}, \mathbf{d}_{\lambda}\right)\right) & =\left(\nabla_{\mathbf{x}} \mathcal{L}(\mathbf{x}, \lambda), \mathbf{d}_{\mathbf{x}}\right)+\left(\nabla_{\lambda} \mathcal{L}(\mathbf{x}, \lambda), \mathbf{d}_{\lambda}\right)+\mu_{2}\left(\mathbf{C}^{T}(\mathbf{x}) \mathbf{h}(\mathbf{x}), \mathbf{d}_{\mathbf{x}}\right) \\
& =\nabla_{\mathbf{x}} \mathcal{L}^{T} \mathbf{d}_{\mathbf{x}}+\nabla_{\lambda} \mathcal{L}^{T} \mathbf{d}_{\lambda}+\mu_{2} \mathbf{h}^{T} \mathbf{C} \mathbf{d}_{\mathbf{x}},
\end{aligned}
$$

where in the last step we have omitted the dependence on $\mathbf{x}$ and $\lambda$ for brevity. With $\mathbf{C d}_{\mathbf{x}}=-\mathbf{h}$ in (C.5) and $-\mathbf{h}^{T} \mathbf{h}<0$, we can set

$$
\mu_{2}>-\frac{\nabla_{\mathbf{x}} \mathcal{L}^{T} \mathbf{d}_{\mathbf{x}}+\nabla_{\lambda} \mathcal{L}^{T} \mathbf{d}_{\lambda}}{\mathbf{h}^{T} \mathbf{C} \mathbf{d}_{\mathbf{x}}}
$$

to ensure the descent condition $\left(\nabla \mathcal{M}_{2},\left(\mathbf{d}_{\mathbf{x}}, \mathbf{d}_{\lambda}\right)\right)<0$. We note that all the quantities $\nabla_{\mathbf{x}} \mathcal{L}, \nabla_{\lambda} \mathcal{L}, \mathbf{d}_{\mathbf{x}}$, $\mathbf{d}_{\lambda}$ and $\mathbf{h}$ are available at the $k$-th iteration $\left(\mathbf{x}^{k}, \lambda^{k}\right)^{T}$ in the SQP method.

\section{C.6 Inexactness of the Solution Process}

The SQP method is very efficient, due to its inherent inexactness of solving the nonlinear KKT conditions at each optimization iteration. In fact, to further enhance the efficiency of the SQP 
method, we can explore another level of inexactness, namely we can use inexact Newton methods to solve the linearized KKT system at each optimization iteration, either in the full space [54] or in the reduced space $[24,71,82]$.

Inexact Newton (or truncated Newton) methods have been very important strategies for solving large scale nonlinear equations $\mathbf{h}(\mathbf{y})=0$. Instead of taking the exact Newton step $\mathbf{d}_{\mathbf{y}}$ by solving the Newton equation $\mathbf{h}\left(\mathbf{y}^{k}\right)+\nabla \mathbf{h}^{T}\left(\mathbf{y}^{k}\right) \mathbf{d}_{\mathbf{y}}=0$, the inexact Newton method accepts the Newton update $\mathbf{d}_{\mathbf{y}}$ as long as the following inexact or relaxed condition is satisfied

$$
\left\|\mathbf{h}\left(\mathbf{y}^{k}\right)+\nabla \mathbf{h}^{T}\left(\mathbf{y}^{k}\right) \mathbf{d}_{\mathbf{y}}\right\| \leq \eta^{k}\left\|\mathbf{h}\left(\mathbf{y}^{k}\right)\right\|
$$

where $\eta^{k}$ is the forcing term $[38,44,45]$. Two strategies for choosing the forcing term $\eta^{k}$ are suggested in [45]: in the first, the forcing term is set as

$$
\eta^{k}=\frac{\left|\left\|\mathbf{h}\left(\mathbf{y}^{k}\right)\right\|_{2}-\left\|\mathbf{h}\left(\mathbf{y}^{k-1}\right)+\nabla \mathbf{h}^{T}\left(\mathbf{y}^{k-1}\right) \mathbf{d}_{\mathbf{y}}^{k-1}\right\|_{2}\right|}{\left\|\mathbf{h}\left(\mathbf{y}^{k-1}\right)\right\|_{2}}
$$

with safeguard

$$
\eta^{k}=\max \left\{\eta^{k},\left(\eta^{k-1}\right)^{1+\sqrt{5} / 2}\right\} \quad \text { when } \quad\left(\eta^{k-1}\right)^{1+\sqrt{5} / 2}>0.1
$$

in the second, the forcing term is set as

$$
\eta^{k}=\gamma\left(\frac{\left\|\mathbf{h}\left(\mathbf{y}^{k}\right)\right\|_{2}}{\left\|\mathbf{h}\left(\mathbf{y}^{k-1}\right)\right\|_{2}}\right)^{\alpha}, \quad \text { where } \gamma \in[0,1] \text { and } \alpha \in(1,2],
$$

with safeguard

$$
\eta^{k}=\max \left\{\eta^{k}, \gamma\left(\eta^{k-1}\right)^{\alpha}\right\} \quad \text { when } \quad \gamma\left(\eta^{k-1}\right)^{\alpha}>0.1
$$

These strategies have been applied successfully to large scale simulations $[26,34,81,110]$, and the advantage of using inexact Newton schemes lies in that it greatly saves computation through acceptting an inexact Newton update $\mathbf{d}_{\mathbf{y}}$ when the current iterate $\mathbf{y}^{k}$ is far from the roots of the nonlinear equations and outside the basins of local quadratic convergence. For the strategy (C.28)(C.29), the $q$-superlinear local convergence can be proved, and for the strategy (C.30)-(C.31), the $q$-quadratic local convergence can be recovered with specifically chosen $\gamma$ and $\alpha$. See [45] for details. 
Inexact Newton methods have been extended to unconstrained optimization problems [39] and constrained optimization problems [24, 26, 54,71,82]. For the full SQP approach, the bound on the forcing term $\eta^{k}$ for solving the nonlinear KKT conditions is given in [26]. For the reduced SQP approach, the bound on $\eta^{k}$ is given in [71] and in [24] for the trust region method and the line search method respectively.

The efficiency gain of introducing the inexactness in solving the linearized KKT conditions is justified by the observation that when the control $\mathbf{u}$ is far from optimal, the quadratic approximation (C.7) defined by the Lagrange-Newton method of the original problem (C.2) is of low quality. Therefore, it is beneficial to use inexact state $\mathbf{y}$, adjoint $\lambda$ and control $\mathbf{u}$ to save computation at the beginning of the optimization scheme. As the control $\mathbf{u}$ approaches optimum, we can reduced the forcing term to recover the superlinear convergence and reduced both the nonlinear constraints and the objective function simultaneously. In complex engineering simulations, such as the CHT process, it is desirable to solve both the state and adjoint equations (C.9)-(C.10) using the inexact Newton method above. 